\title{
Thermal Evolution and Magnetic Field Generation in Terrestrial Planets and Satellites
}

\author{
Doris Breuer • Stephane Labrosse • Tilman Spohn
}

Received: 20 May 2009 / Accepted: 2 November 2009 / Published online: 12 January 2010

(C) Springer Science+Business Media B.V. 2010

\begin{abstract}
Of the terrestrial planets, Earth and Mercury have self-sustained fields while Mars and Venus do not. Magnetic field data recorded at Ganymede have been interpreted as evidence of a self-generated magnetic field. The other icy Galilean satellites have magnetic fields induced in their subsurface oceans while Io and the Saturnian satellite Titan apparently are lacking magnetic fields of internal origin altogether. Parts of the lunar crust are remanently magnetized as are parts of the crust of Mars. While it is widely accepted that the magnetization of the Martian crust has been caused by an early magnetic field, for the Moon alternative explanations link the magnetization to plasma generated by large impacts. The necessary conditions for a dynamo in the terrestrial planets and satellites are the existence of an iron-rich core that is undergoing intense fluid motion. It is widely accepted that the fluid motion is caused by convection driven either by thermal buoyancy or by chemical buoyancy or by both. The chemical buoyancy is released upon the growth of an inner core. The latter requires a light alloying element in the core that is enriched in the outer core as the solid inner core grows. In most models, the light alloying element is assumed to be sulfur, but other elements such as, e.g., oxygen, silicon, and hydrogen are possible. The existence of cores in the terrestrial planets is either proven beyond reasonable doubt (Earth, Mars, and Mercury) or the case for a core is compelling as for Venus and the Moon. The Galilean satellites Io and Ganymede are likely to have cores judging from Galileo radio tracking data of the gravity fields of these satellites. The case is less clear cut for Europa. Callisto is widely taken as undifferentiated or only partially differentiated, thereby lacking an iron-rich core. Whether or not Titan has a core is not known at the present time. The terrestrial planets that do have magnetic fields either have a well-established inner core with known radius and density such as Earth or are widely agreed to have an inner core such as Mercury. The absence of an inner core in Venus, Mars, and the Moon (terrestrial bodies that lack fields) is not as well established although considered likely. The composition of the Martian core may be close
\end{abstract}

D. Breuer $(\bowtie) \cdot$ T. Spohn

DLR, Institute of Planetary Research, Rutherfordstr. 2, 12489 Berlin, Germany

e-mail: doris.breuer@dlr.de

S. Labrosse

Laboratoire des sciences de la Terre, ENS Lyon, 46 Allée d'Italie, 69364 Lyon Cedex 07, France 
to the $\mathrm{Fe}-\mathrm{FeS}$ eutectic which would prevent an inner core to grow as long as the core has not cooled to temperatures around 1500 Kelvin. Venus may be on the verge of growing an inner core in which case a chemical dynamo may begin to operate in the geologically near future. The remanent magnetization of the Martian and the lunar crust is evidence for a dynamo in Mars' and possibly the Moon's early evolution and suggests that powerful thermally driven dynamos are possible. Both the thermally and the chemically driven dynamo require that the core is cooled at a sufficient rate by the mantle. For the thermally driven dynamo, the heat flow from the core into the mantle must by larger than the heat conducted along the core adiabat to allow a convecting core. This threshold is a few $\mathrm{mW} \mathrm{m}^{-2}$ for small planets such as Mercury, Ganymede, and the Moon but can be as large as a few tens $\mathrm{mW} \mathrm{m}^{-2}$ for Earth and Venus. The buoyancy for both dynamos must be sufficiently strong to overcome Ohmic dissipation. On Earth, plate tectonics and mantle convection cool the core efficiently. Stagnant lid convection on Mars and Venus are less efficient to cool the core but it is possible and has been suggested that Mars had plate tectonics in its early evolution and that Venus has experienced episodic resurfacing and mantle turnover. Both may have had profound implications for the evolution of the cores of these planets. It is even possible that inner cores started to grow in Mars and Venus but that the growth was frustrated as the mantles heated following the cessation of plate tectonics and resurfacing. The generation of Ganymede's magnetic field is widely debated. Models range from magneto-hydrodynamic convection in which case the field will not be self-sustained to chemical and thermally-driven dynamos. The wide range of possible compositions for Ganymede's core allows models with a completely liquid near eutectic $\mathrm{Fe}-\mathrm{FeS}$ composition as well as models with $\mathrm{Fe}$ inner cores or cores in with iron snowfall.

Keywords Magnetic field generation · Thermal evolution · Terrestrial planets · Satellites

\section{Introduction}

Magnetic fields of internal origin are widely held to be characteristic of planets and at least the large satellites (for a recent review, see Connerney 2007). Magnetized meteorites suggest that even planetesimals may have had their own self-generated fields (Weiss et al. 2008). Of the terrestrial planets and satellites, Mercury, Earth, and Ganymede are known to have largely dipolar internally generated magnetic fields although there has been some debate of whether or not Ganymede's field is really self-sustained (e.g., Crary and Bagenal 1998). Mars has a remanently magnetized crust in mostly the southern hemisphere (Acuña et al. 1998). This remanent magnetization is evidence for an earlier self-generated field (Connerney et al. 2004). The Moon also has crust units with remanent magnetization, some of them located suspiciously close to the antipodes of major impact basins (Mitchell et al. 2008). While the magnetization may be taken as evidence of an ancient magnetic field for the Moon, alternative explanations have been given. One of the alternative explanations invokes plasma generated upon impact where the great basins are formed (e.g., Hood and Vickery 1984). It has been argued that Venus should have had an early magnetic field, also (Stevenson et al. 1983). The high surface temperature would have annealed any remanent magnetization since the temperature is above the Curie temperature (the magnetic blocking temperature) of most remanently magnetizable minerals.

Generation of a magnetic field requires an electrically conducting shell within a planet and motion within that shell. In the terrestrial planets and by the satellites, this region is agreed to be the fluid iron-rich core at the center. For recent reviews of Earth and planetary 
dynamos and convection in the core, see Roberts (2007), Christensen and Wicht (2007), Jones (2007), and Busse and Simitev (2007). There may be a solid inner core; the growth of which may provide a buoyancy flux that may drive the dynamo. The buoyancy in this case derives from a difference in composition between the solid inner core and the fluid outer core. Light alloying elements such as sulfur and oxygen (most models consider sulfur) tend to be expelled from the solidifying core and concentrate in the fluid outer core. In addition to a chemical buoyancy, flux from the inner core or alternatively to that buoyancy flux, thermal buoyancy may drive the flow. The thermal buoyancy results from a sufficiently large temperature difference between the core and the rocky mantle surrounding the core.

Regardless of whether chemical or thermal buoyancy drive core convection, the existence of a dynamo and the magnetic field strength are strongly dependent on the heat transfer rate through the mantle of the planet. The thermal buoyancy flux is directly related to the heat flow extracted by the mantle from the core. For a chemically driven dynamo, the rate of inner core growth and, therefore, the rate of buoyancy release depends on the core cooling rate and, therefore, on the heat flow from the core. The heat flow from the core is directly dependent on the vigor of convection in the mantle and the mantle heat transfer rate. The latter can be calculated as a function of time from planet thermal history models.

Thermal history models have been pioneered by Schubert and others (e.g., Schubert et al. 1979 ) in the late 70s and early 80s. These models use a parameterization of the heat transfer rate by convection through the planetary mantle and are usually based on a relation between the Nusselt number - a dimensionless measure of heat flow-and the Rayleigh numbera dimensionless measure of convective vigor. The boundary layer theory has been used to establish that relation (e.g., Schubert et al. 2001) but empirical data have also been used (e.g., Booker and Stengel 1978). More recently, it has been recognized that the classical boundary layer theory must be modified to include the effects of stagnant lids in fluids with strong temperature dependent viscosities (Davaille and Jaupart 1993; Moresi and Solomatov 1995; Grasset and Parmentier 1998).

Stevenson et al. (1983) were the first to discuss the thermal evolution of the core and the dynamo for the terrestrial planets. These authors included a discussion of the growth of an inner core and a simple estimate of the magnetic field strength based on the power dissipated in the core. Stevenson et al. concluded that the Earth should have had a thermally driven dynamo in its early evolution when the core was completely liquid. Freeze out of an inner core started after a few billion years of cooling and the dynamo switched from thermally driven to chemically driven. Venus, as these authors concluded, would have had an early magnetic field driven by a thermal dynamo that ceased to operate as the heat flow from the core dropped below the value that would also be supported by heat conduction along the core adiabat. A similar explanation was offered to explain the lack of a magnetic field of Mars that was later refined by Schubert and Spohn (1990). Mercury has a magnetic field because the thin mantle cools the core rapidly enough to allow the growth of an inner core.

These early models have not included the effects of stagnant lids on the cooling of the planet. In the late 90s of the previous century, the magnetism of the Galilean satellites was explored by the Galileo spacecraft. At the time of this writing, the Cassini mission is actively exploring the Saturnian satellites. In addition, new laboratory data on the melting relations of iron and iron alloys have become available and first super-Earth Exoplanets have been detected. It is thus timely to revisit the subject of the magnetism of the terrestrial planets and to review the progress of research in the field since Stevenson et al. (1983), revise their findings where necessary, and include a discussion of the Moon, the Galilean satellites, and Titan. In an outlook, we briefly speculate on the magnetic properties of Earth-like Exoplanets. 


\section{Structure Sets the Stage}

It is widely accepted that the source region of self-generated magnetic fields in terrestrial planets and satellites is the iron-rich core. It is also widely accepted that the terrestrial planets are differentiated into iron-rich cores, silicate largely peridotitic mantles, and basaltic crusts. (The Earth's crust is divided between a granitic continental crust and a basaltic oceanic crust.) For the satellites, the situation is less well established. For instance, it is still debated whether or not the Moon and Titan have iron-rich cores (see Sohl and Schubert 2007, and Hussmann et al. 2007 for reviews) and the Jovian satellite Callisto is widely agreed to be undifferentiated or only partly differentiated.

The interior structure of a planet can be deduced from geodetic and geophysical data (gravity, topography, rotation, seismic, and magnetic field) but cosmochemical data are often providing conclusive evidence when the geophysical data are not allowing unique solutions. The recently published Volume 10 of the Treatise on Geophysics collects a number of excellent reviews of relevance on planetary geophysics and geodesy (e.g., Sohl and Schubert 2007; Wieczorek 2007; Van Holst 2007; Lognonne and Johnson 2007; Connerney 2007; Hussmann et al. 2007; Breuer and Moore 2007). Unfortunately, seismological data are only available for the Earth and the Moon. Although plans for establishing seismic networks on other terrestrial planets, in particular on Mars, have been discussed for decades, network missions have yet to be selected and implemented by the space agencies.

The most useful and widely available data for determining the core radius of a planet or satellite are the mass $M$, the radius $r_{\mathrm{p}}$, and the quadrupole moment $J_{2}$ of the gravity field. For a planet in hydrostatic equilibrium, the Radau-Darwin relation holds and the latter is directly proportional to the moment of inertia $C$ of the planet about its rotational axis

$$
J_{2} \sim \frac{C}{M r_{\mathrm{p}}^{2}}
$$

(see, e.g., Zharkov and Trubitsyn 1978; Hubbard 1984). Since the moment of inertia $C$ is the integral over mass times distance from the rotation axis squared, $C / M r_{\mathrm{p}}^{2}$ is a dimensionless measure of the mass distribution in the planet. It can be compared to the average moment of inertia of a spherical model planet $I$

$$
I \equiv \int_{0}^{M} r^{2} \mathrm{~d} m,
$$

where $r$ is the radial distance of the mass element $\mathrm{d} m$ from the rotation axis. The dimensionless average moment of inertia $I / M r_{\mathrm{p}}^{2}$ is related to $C / M r_{\mathrm{p}}^{2}$ via

$$
\frac{I}{M r_{\mathrm{p}}^{2}}=\frac{C}{M r_{\mathrm{p}}^{2}}-\frac{2}{3} J_{2}
$$

(Sohl et al. 2005). $I / M r_{\mathrm{p}}^{2}$ is the constraint to be used for interior structure modeling.

If the planet is not in hydrostatic equilibrium, $J_{2}$ is proportional to a linear combination of the principal moments of inertia and additional data such as the precession rate of the planet must be used to calculate $C$ and $I$. In any case, a reasonable constraint can only be derived for a fast enough rotator for which $J_{2}$ is a clear measure of the dynamic flattening of the planet. This can be estimated by comparing the value of $J_{2}$ with the ratio between 
Table 1 Planetary radii, moment of inertia factors, core radii, and densities of terrestrial planets and satellites. The planetary radii are from Landolt-Börnstein (2009), the moment of inertia factor for the Earth is from Lodders and Fegley (1998), for Mars and the Moon from Sohl and Schubert (2007), for the Galilean satellites from Schubert et al. (2004). The core radii and densities are discussed in the text. Note that the value for Venus is a representative value estimated by rescaling the Earth to Venus

\begin{tabular}{llllll}
\hline & $r_{\mathrm{p}}[\mathrm{km}]$ & $C / M r_{\mathrm{p}}^{2}$ & $I / M r_{\mathrm{p}}^{2}$ & $r_{\mathrm{c}}[\mathrm{km}]$ & $\rho_{\mathrm{c}}\left[10^{3} \mathrm{~kg} \mathrm{~m}^{-3}\right]$ \\
\hline Earth & 6371.01 & 0.3307 & 0.3293 & $3480(\mathrm{oc})$ & 10.9 \\
& & & & $1220(\mathrm{ic})$ & 12.9 \\
Venus & 6052.878 & $?$ & $?$ & 3089 & 11 \\
Mars & 3390.500 & $0.3650 \pm 0.0012$ & 0.3635 & $1400-1900$ & $5.5-8.5$ \\
Mercury & 2439.700 & $?$ & $?$ & $1700-2300$ & $5.1-8.1$ \\
Moon & 1737.064 & $0.3931 \pm 0.0002$ & 0.3930 & $150-400$ & $5.1-8.1$ \\
Io & 1821. & $0.37824 \pm 0.00022$ & 0.3770 & $650-950$ & $5.1-8.1$ \\
Europa & 1560. & $0.346 \pm 0.005$ & 0.3460 & $200-700$ & $5.1-8.1$ \\
Ganymede & 2634. & $0.3115 \pm 0.0028$ & 0.3114 & $650-900$ & $5.1-8.1$ \\
Callisto & 2400. & $0.3549 \pm 0.0042$ & 0.3549 & No & \\
Titan & 2575. & $?$ & $?$ & $0-800$ & $5.1-8.1$ \\
\hline
\end{tabular}

the centrifugal acceleration measured at the equator of the planet to the acceleration due to gravity at the equator

$$
q \equiv \frac{\omega^{2} r_{\mathrm{p}}^{3}}{G M} .
$$

For a planet in hydrostatic equilibrium, $J_{2}$ is about $0.5 q$. For Venus, $J_{2}$ is two orders of magnitude larger than $q$. Reliable data are available for the Earth and Mars. For the Galilean satellites, the Galileo mission has provided values of $J_{2}$ and $J_{22}$. Values of $C / M R^{2}$ haven been derived from these under the assumption of hydrostatic equilibrium and interior structure models have been calculated. Unfortunately, no data are yet available for Titan.

In the following, we will briefly summarize what is known about the core and inner core radii and densities for the terrestrial planets and satellites. Parameter values have been collected in Table 1.

For the Earth, the combined evidence from seismology and gravity (Dziewonski and Anderson 1981; Kennett et al. 1995) gives a core of $3480 \mathrm{~km}$ radius and a mass of $1.932 \times 10^{24} \mathrm{~kg}$. The core is layered with a liquid outer core and a solid inner core. The inner core has a radius of $1220 \mathrm{~km}$. The average densities of the outer and inner cores as given by Olson (2007) are $10.9 \times 10^{3} \mathrm{~kg} \mathrm{~m}^{-3}$ and $12.9 \times 10^{3} \mathrm{~kg} \mathrm{~m}^{-3}$, respectively. The density increase across the boundary is $0.5 \times 10^{3} \mathrm{~kg} \mathrm{~m}^{-3}$ to $1 \times 10^{3} \mathrm{~kg} \mathrm{~m}^{-3}$ (Souriau 2007) and is attributed to a difference in the specific volumes of solid and liquid iron and to a difference in chemistry between the outer and inner cores. The outer core is widely held to contain about 10 weight- $\%$ of light alloying elements with oxygen, sulfur, and silicon as the most likely candidates. The boundary between the cores is the liquidus of the alloy and the light alloying elements are expelled from the inner core as the latter grows through core freezing (see, e.g., Vocadlo 2007, and references therein).

The interior structure of Venus is much less well constrained. There are no seismic data available for the planet. The technological challenge of placing seismometers on the surface of the planet is overwhelming given the average surface temperature of about $740 \mathrm{~K}$, the pressure of 95 bar, and the presence of highly corrosive $\mathrm{SO}_{2}$ in the atmosphere. The gravity 
field and the topography have been measured by Doppler tracking of the Magellan spacecraft (Sjogren et al. 1997) and by radar altimetry (Rappaport et al. 1999) but the moment of inertia factor cannot be reliably estimated because of the slow rotation rate of the planet and the dominance of nonhydrostatic effects on the $J_{2}$ coefficient. It is widely assumed (see Sohl and Schubert 2007, for a review) that the interior structure of Venus is Earth-like with a core, a mantle and a crust. Since the intrinsic density of Venus is smaller than that of Earth (BVSP 1981), the core is relatively small; 0.51 planetary radii as compared with 0.55 planetary radii for Earth. Doppler tracking data of the Magellan spacecraft suggest that the core is liquid; at least that there is a liquid outer core (Yoder 1997). Stevenson et al. (1983) have suggested that the Venusian core is lacking a growing inner core to explain the absence of a presentday magnetic field. Their rescaling of the Earth to the smaller size and mass of Venus while accounting for its high surface temperature resulted in a central pressure and temperature similar to the temperature and pressure at the surface of Earth's present day inner core. The core of Venus-according to that model-may start freezing within the next few million years.

The interior structure of Mars is better constrained although useful seismic data have not been collected from the surface of that planet as well. The Viking landers in the 70s did carry seismometers but these did not deliver useful data. Thus, the interior structure of Mars is mostly constrained by gravity, topography, and rotation data and by the chemistry of the SNC meteorites. Highly accurate gravity and topography data have been obtained using laser altimetry and two-way Doppler tracking of the Mars Global Surveyor (MGS) spacecraft (Smith and Zuber 1996; Zuber et al. 2000; Lemoine et al. 2001). The parameters describing the orientation of the rotation axis and its variation with time have been obtained by combining Viking and Mars Pathfinder tracking data (Folkner et al. 1997) and later by including MGS and Mars Odyssey data (Konopliv et al. 2006). The best available value for $C / M r^{2}$ is $0.3650 \pm 0.0012$ (Yoder et al. 2003) from which a value of $I / M r^{2}=0.3635 \pm$ 0.0012 has been derived (Sohl et al. 2005). The latter value also includes minor corrections for the Tharsis bulge. The improved values of the moment of inertia factors are smaller than previous values and suggest stronger concentrations of the mass toward the center than in, e.g., the models of Sohl and Spohn (1997). However, due to the non-uniqueness of any interpretation of gravity data and due to uncertainties in crust thickness and crust, mantle, and core densities the value of the Martian core radius is still uncertain. Most published models (e.g., Sohl and Spohn 1997; Sohl et al. 2005; Bertka and Fei 1997) have core radii between 1400 and $1900 \mathrm{~km}$ and core densities between 5500 and $8400 \mathrm{~kg} \mathrm{~m}^{-3}$. Models that satisfy the chemistry of the SNC meteorites in addition to the gravity data tend to have core radii around $1600 \mathrm{~km}$ and densities of about $6000 \mathrm{~kg} \mathrm{~m}^{-3}$. These models suggest that the core of Mars has about 14 wt.-\% sulfur, which is close to the eutectic Fe-FeS composition at Martian core pressure.

The state of the Martian core is not known with certainty, as well as the answer to the question of whether or not there is a solid inner core. However, analysis of 3 years of MGS tracking data suggests that at least an outer layer of the core is liquid (Yoder et al. 2003). Schubert and Spohn (1990) have argued for the simplest explanation for the absence of a present-day magnetic field to be the absence of an inner core driving a chemical dynamo. The results of the laboratory measurements of Fei et al. (1997) of the core liquidus temperature together with estimates of the actual core temperature by, e.g., Williams and Nimmo (2004) and Breuer and Spohn (2006) also suggest that the Martian core is entirely liquid.

The deep interior structure of Mercury is about as well constrained from geophysical data as that of Venus. The high density of the planet suggests that the core is large, about $1800 \mathrm{~km}$ radius or $80 \%$ of the radius of the planet (e.g., Siegfried and Solomon 1974; BVSP 
1981; Spohn et al. 2001a). Harder and Schubert (2001) have argued for an even larger core that would require a substantial amount of volatiles in the core. Most cosmochemists (e.g., Woods et al. 1981), however, would argue for a refractory composition of the planet and a mostly iron core. The existence of a magnetic field measured by the Mariner 10 spacecraft (Ness et al. 1975) suggests that there is at least an outer liquid core and the thin mantle of the planet suggests that cooling has resulted in a substantial solid inner core (e.g., Stevenson et al. 1983).

Peale $(1976,1988)$ has outlined a method that would allow calculating the moment of inertia factor of the planet without having to assume hydrostatic equilibrium. The method would also allow proving the existence of a liquid outer core and an estimate of the moment of inertia factor of the core itself. The latter can be used to constrain the size of the inner core. The model is based on the observation that Mercury occupies a resonant Cassini state with the rotation axis and the normal to the ecliptic plane being coplanar. Measurement of the amplitude of physical libration together with the obliquity and the gravity field coefficients $J_{2}$ and $J_{22}$ will allow the determination of $C / M r^{2}$ as well as the ratio between $C_{\mathrm{m}}$ and $C$ where $C_{\mathrm{m}}$ is the moment of inertia attributed to the solid part of the planet. The ratio is 0.5 and smaller in case there is a liquid core or outer core shell while it will be about 1 in case the planet is completely solid. Margot et al. (2007) have recently succeeded in measuring the libration rate from earthbound radar speckle observations. The data already suggest a liquid core layer. The gravity coefficients are expected to be measured by the Messenger (Solomon et al. 2001) and BepiColombo (Anselmi and Scoon 2001) missions.

The lunar interior has been probed with seismometers but an iron core has not been detected. The geometry of the seismic stations on the Moon and the foci of deep lunar quakes did not allow for rays that probed the core to be recorded (for a recent review of planetary and lunar seismology, see Lognonne and Johnson 2007). The seismic data are consistent with a core of 170 to $360 \mathrm{~km}$ (Nakamura et al. 1974). The averaged moment of inertia factor $I / M r^{2}$ has been determined with improved accuracy from Doppler tracking of the Lunar Prospector spacecraft and from lunar laser ranging data by Konopliv et al. (2001) to $0.3931 \pm 0.0002$. This value together with the mass of the planet suggests a core radius of $220 \mathrm{~km}$ to $450 \mathrm{~km}$. This range is consistent with independent lunar electromagnetic induction data (Hood et al. 1999) and joint inversions of seismic and gravity data (e.g., Khan et al. 2004) and with the chemical models of Kuskov and Kronrod (2001). The densities consistent with the radii given above vary between roughly $5100 \mathrm{~kg} \mathrm{~m}^{-3}$ and $8100 \mathrm{~kg} \mathrm{~m}^{-3}$. The former value is typical for a $\mathrm{Fe}-\mathrm{FeS}$ eutectic composition while the latter suggest a pure iron core. The lunar laser ranging data suggest that the core is liquid (Yoder 1981; Dickey et al. 1994; Williams et al. 2001).

The Galileo mission has provided useful gravity data on the interior structure of the Galilean satellites. For discussions and overviews, see Sohl et al. (2002), Schubert et al. (2004), and Hussmann et al. (2007). Two-way Doppler tracking of the spacecraft has provided values for the $J_{22}$ quadrupole gravity field coefficient from near-equatorial fly-bys. Assuming hydrostatic equilibrium, $J_{2}$ can be calculated from

$$
J_{2}=\frac{10}{3} J_{22}
$$

For Io, polar fly-bys have allowed an independent determination of $J_{2}$. The measured values of $J_{2}$ and $J_{22}$ are consistent with hydrostatic equilibrium. Note, however, that (5) is required for equilibrium but not sufficient to prove it. Again, assuming hydrostatic equilibrium, values of $C / M r^{2}$ have been calculated for Io (Anderson et al. 2001a), Europa (Anderson et al. 1998), Ganymede (Anderson et al. 1996), and Callisto (Anderson et al. 2001b). The core 
radii for Io vary between $650 \mathrm{~km}$ for a purely iron core and $950 \mathrm{~km}$ for a Fe-FeS eutectic core (Schubert et al. 2004). The densities would be $8090 \mathrm{~kg} \mathrm{~m}^{-3}$ and $5150 \mathrm{~kg} \mathrm{~m}^{-3}$, respectively. The large availability of sulfur in the outer parts of the solar system and the large amounts of sulfur on Io's surface (e.g., Lewis 1982) favors sulfur-rich models of the satellite's core although it must be said that the composition of the cores of the Galilean satellites is not known.

The core radii of the icy satellites of Jupiter (Europa, Ganymede, and Callisto) are even less certain because the significant share of ice as a third component in addition to rock and iron adds to the nonuniqueness of the models. Estimates of the core radius of Europa vary between roughly 200 and $700 \mathrm{~km}$ depending on the composition of the core and the thickness of the ice layer (Sohl et al. 2002). The former value is consistent with an iron rich core while the latter is consistent with a eutectic Fe-FeS core and densities as given for Io above. For Ganymede, core radii vary between $650 \mathrm{~km}$ and $900 \mathrm{~km}$, similar to the values for Io and with similar values for the range of core densities (Sohl et al. 2002). The ice shell in these models is $900 \mathrm{~km}$ thick which supports a rule-of-thumb structural model for Ganymede as an Io plus an ice shell (see also Kuskov and Kronrod 2001). The presence of a magnetic field of deep internal origin for Ganymede suggests that at least an outer shell of Ganymede's core is liquid.

Callisto is unusual among the larger satellites of Jupiter. The moment of inertia data suggests that the satellite is incompletely differentiated and, therefore, lacks a core (Anderson et al. 2001a; Sohl et al. 2002; Schubert et al. 2004). Nagel et al. (2004) have provided a model for the incomplete differentiation of the satellite. It has been suggested that Titan may also be incompletely differentiated (e.g., Lunine and Stevenson 1987; Grasset et al. 2000) although possibly to a different degree. Interpretation of the tracking data of the Cassini spacecraft is made difficult by the large fly-by distance and the effects of the dense atmosphere of the satellite and a value for the moment of inertia factor is still not available. Models must be based on the mass and radius alone and allow wide ranges of interior structure between models similar to Callisto and models that are similar to Ganymede with a substantial core.

\section{The Core Is the Stage}

The magnetic field of a terrestrial planet is most readily maintained against losses by diffusion by flow in its iron-rich core. This statement results from the induction equation for a conducting fluid that reads

$$
\frac{\partial \mathbf{B}}{\partial t}=\nabla \times(\mathbf{u} \times \mathbf{B})-\nabla \times\left(\eta_{\mathrm{m}} \nabla \times \mathbf{B}\right),
$$

with $\eta_{\mathrm{m}}$, the magnetic diffusivity, $\mathbf{B}$ the magnetic induction, and $\mathbf{u}$ the fluid velocity. (Vector quantities are bold face.) In the absence of flow, this equation reduces to a diffusion equation which implies that any initial magnetic field would decay on a timescale of $d^{2} / \eta_{\mathrm{m}}$, with $d$ the characteristic length-scale on which the field varies. With $\eta_{\mathrm{m}}$ approximately $5 \times 10^{-6} \mathrm{~m}^{2} \mathrm{~s}^{-1}$ a timescale of the order of $15 \mathrm{kyr}$ is derived for the Earth taking the core radius as the length scale. This time is much shorter than the lifetime of the Earth's magnetic field. As a consequence, a sufficiently vigorous fluid flow is required to maintain dynamo action. The magnetic Reynolds number, $R_{\mathrm{m}}=U d / \eta_{\mathrm{m}}$ measures the ratio between the two terms on the right-hand side of the induction equation. Some flows are known to not generate magnetic fields irrespective of the value of $R_{\mathrm{m}}$ (these are subject to so called antidynamo theorems), 
but for many flows dynamo action occurs whenever $R_{\mathrm{m}}$ exceeds the critical value of order 100. The magnetic diffusivity is linked to the electrical conductivity $\sigma$ by $\eta_{\mathrm{m}}=1 / \mu_{0} \sigma$ where $\mu_{0}$ is the magnetic permeability. Since rocky planet mantles are insulators, their magnetic Reynolds numbers are too small to allow dynamo action which leaves the liquid cores as the source regions for the magnetic fields.

Several driving forces are possible to maintain the flow in the core. The most likely ones, and the only ones considered in this paper, are convection flows. The other main possibility is mechanical stirring caused either by a change in the rotation vector of the mantle or by tidal deformation of the mantle which can lead to elliptic instabilities (Kerswell 2002). Precession driven dynamos fall in the first category and the flow also comes from an elliptic instability. Tilgner (2005) showed that dynamo action can be produced by such a flow, although this requires the basic state to be neutrally stratified. In a planet core, this implies that the temperature follows an isentrope and that the composition is uniform, which can only be obtained through vigorous convection. Whether or not precession plays a role, it must be considered as an effect additional to convection.

\subsection{Core Heat and Solute Transfer}

Convection is the major player in the dynamics of planetary interiors and our understanding of this problem benefits from the important physics literature on the subject. A major difference between well-controlled convection experiments and planetary interiors is that in the latter case, the energy available to drive the flow comes from the long term evolution of the planet and the decay of radioactive isotopes. Therefore, the driving power is constantly evolving and an assumption of statistical steady state is not warranted. Fortunately, the low viscosity of liquid iron ensures a dynamics that is much faster than the evolution timescale of the planet, mostly controlled by sluggish convection in the solid mantle and separation of timescales is usually assumed to hold. The short term dynamics are considered to maintain the core in an average state close to isentropic and well mixed and the long term evolution of this average state provides the energy to drive convection.

\subsubsection{Convection}

Broadly defined, convection is a transport mechanism that occurs by macroscopic motion of fluid, independently of the origin of the flow. In planetary interiors, the flow originates from unstable density stratifications maintained by buoyancy sources at the boundariesthe planet's surface or the surface of its core, and in the bulk interior. When both compositional and temperature variations contribute to buoyancy, thermo-compositional or thermochemical convection arises. The motion resulting from the initially unstable stratification redistributes this buoyancy and tends to erase the initial stratification. In the absence of buoyancy sources that are somehow maintained, the system simply comes to rest after sufficient redistribution has been attained. The planetary cores can convect beyond an initial overturn because buoyancy sources are maintained by the slow thermal evolution of the planet.

In the case of planetary cores, two types of buoyancy contribute to convective motion, thermal and compositional buoyancy. Thermal buoyancy results from thermal expansion in a hot core in contact with a colder mantle. In addition, the core may be heated by the decay of radioactive elements. Compositional buoyancy results from the core being an alloy of metal (iron and nickel) and some lighter elements; the most discussed ones being S, Si, and O (Poirier 1994) and, in the most classical scenario, the crystallization of an inner core that 
is denser than the liquid outer core. Conservation of chemical species implies a flux of light elements from the bottom of the outer core which provides chemical buoyancy able to drive flow in the liquid outer core. The origin of this chemical buoyancy and the crystallization of the inner core is therefore linked to the cooling of the core.

As a mode of heat or solute transfer, convection competes with diffusion. In the case of a solute, diffusion is very inefficient and can usually be neglected. On the other hand, metals are good thermal conductors and thermal diffusion may play an important role in planetary cores. Heat transfer in a solid inner core is thought to occur by diffusion (Yukutake 1998). But even in liquid outer cores, diffusion is relevant in boundary layers and in the bulk of the interior as heat conduction along the isentropic temperature gradient.

In planetary cores, convection is assumed to maintain an isentropic (or adiabatic) temperature profile that can be calculated from

$$
\frac{\mathrm{d} T}{\mathrm{~d} z}=\frac{\alpha T g}{C_{\mathrm{p}}}
$$

with $T$ denoting temperature, $z$ depth, $\alpha$ the thermal expansion coefficient, $g$ the acceleration of gravity, and $C_{\mathrm{p}}$ the specific heat at constant pressure. In fact, the isentropic temperature gradient has to be exceeded for convection to occur. Generally speaking, thermal convection occurs when the imposed temperature gradient exceeds a minimum value, or equivalently when a suitably defined dimensionless number, the Rayleigh number, is larger than a critical value. The exact definition of this number depends on the mode of heating which will be discussed further below in Sect. 4.1.1. In the case of compressible fluids, the relevant temperature gradient is that in excess of the isentropic value (e.g., Jeffreys 1930; Spiegel and Veronis 1960; Hewitt et al. 1975). Because of the low viscosity of liquid iron, a very small excess temperature suffices to start vigorous convection. With the thermal conductivity of iron typically being ten times larger than that of rock (Stacey and Anderson 2001), the heat flow along the isentropic temperature profile (hereafter termed the isentropic heat flow) can be a large fraction of the total heat flow out of the core. For example, the isentropic heat flow at the top of the Earth's core is thought to be about 5-7 TW or about 8 to $12 \mathrm{~mW} \mathrm{~m}^{-2}$ (e.g., Labrosse 2003), a nonnegligible fraction of the total heat loss of the Earth of 46 TW (Jaupart et al. 2007).

\subsubsection{Diffusion}

Even when convection dominates in the bulk of the core, the vertical velocity has to go to zero at the horizontal boundaries. This means that radial diffusion near the boundary must balance the convective heat flow and leads to the formation of boundary layers where the radial temperature gradient is larger than in the interior. However, the thicknesses of these boundary layers are inversely proportional to the Rayleigh number and in the core are usually thought to be quite small. An estimate can be obtained for the case of the Earth's core by using the secular variation of the magnetic field. The flow velocity at the top of the core can be estimated from the secular variation of the Earth's magnetic field (see Hulot et al. 2002 , for a recent calculation) and the typical velocity, about $10^{-4} \mathrm{~m} \mathrm{~s}^{-1}$, allows an estimate of the total buoyancy that drives it, that is, the relative density anomalies $\delta \rho / \rho \sim 10^{-9}$ (e.g., Braginsky and Roberts 1995; Labrosse et al. 1997). Assuming these density anomalies to result from temperature anomalies and using $\alpha \sim 10^{-5} \mathrm{~K}^{-1}$, we get $\delta T \sim 10^{-4} \mathrm{~K}$. These small lateral temperature variations result from the destabilization of boundary layers and the total temperature differences across them are of the same order of magnitude. Assuming a 
super-isentropic heat flow at the core-mantle boundary (CMB) of, say, $\delta Q=4 \pi r_{\mathrm{c}}^{2} k \delta T / \delta z \approx$ $1 \mathrm{TW}$ ( $r_{\mathrm{c}}$ being the radius of the core), one can obtain a typical thickness for the boundary layer of $\delta z \sim 0.8 \mathrm{~m}$. This shows that the boundary layers that must exist in the core are very thin with very small total super-isentropic temperature differences. The reason is the very low viscosity of liquid iron (Poirier 1988; Terasaki et al. 2001) which likely applies to other planetary cores as well. Note that the boundary layers in the mantle are many orders of magnitude thicker as will be addressed further below.

Thermal diffusion can play an important role in wider regions of the core if the heat flow at the CMB is lower than the isentropic heat flow. For the Earth, the heat flow from the core may be approximately equal to the isentropic heat flow and thermal convection at the top of the core is not necessarily guaranteed. This problem has been recognized by earlier workers in the field (Loper 1978a, 1978b; Stevenson 1983) who proposed that compositional convection would act against thermal stratification in this case and maintain the average state close to isentropic. This requires compositional convection to transport downward the excess heat conducted along the isentrope and would lead to a much lower efficiency of the dynamo. Labrosse et al. (1997) and Lister and Buffett (1998) proposed instead that a stably stratified layer of about $100 \mathrm{~km}$ thickness through which heat would be transferred by thermal diffusion could form at the top of the core. Even if the total heat flow at the CMB were above the isentropic value, there could be regions in the core where a local thermal stratification would develop and their effect on the global core dynamics could be important. Also, because of fluctuations of the CMB heat flow imposed by the mantle on a time-scale of about 400 Myr (e.g., Grignè et al. 2005; Nakagawa and Tackley 2005; Labrosse and Jaupart 2007), one may speculate about periods in time during which thermal stratification would expand from the top of the core into the deeper interior and periods where it would shrink (Buffett 2007). The effect of such a scenario on the generation of the magnetic field has not been fully considered yet in the case of the Earth and it could be important for other planets as well.

Christensen (2006) proposed a similar scenario for Mercury, where inefficient heat transport by the mantle makes the CMB heat flow small. The main effect of such a stable layer at the top of the core is to filter the magnetic field that is produced underneath. The magnetic field observed outside of the planet is then smaller and of larger scale than that in the convectively active region.

Compositional stratification is also possible at the top of the core through the accumulation of light elements (Braginsky 1993, 2006). Braginsky (2006) calls this layer the stratified ocean of the core because the positive compositional buoyancy of such a layer compared to its thermal buoyancy would be similar to the negative buoyancy of the ocean in the atmosphere. It is difficult to test the existence of this layer but it would help to explain an incomplete chemical equilibration between the core and the mantle (Labrosse et al. 2007) which otherwise would result in a core containing too many light elements to be reconciled with seismological observations (Asahara et al. 2007). If this layer is formed by extraction (possibly exsolution, see below) of light elements from the bulk of the core, an additional buoyancy source would be provided that would help drive the dynamo.

\subsection{Energy and Entropy Balances}

In this section, the global balances for energy and entropy are written for planetary cores, in the case of an Earth-like structure, that is with an inner core growing from the center outward. As discussed below, more exotic situations can be encountered in other planets but their thermodynamics have not been completely worked out yet. 
The thermal evolution of planetary cores is controlled by an equation for energy conservation in which the total heat loss $Q_{\mathrm{CMB}}$, set by mantle convection (Sect. 4), is balanced by the sum of several sources:

$$
Q_{\mathrm{CMB}}=Q_{\mathrm{ICB}}+Q_{\mathrm{C}}+Q_{\mathrm{L}}+E_{\xi}+Q_{\mathrm{H}}
$$

with $Q_{\text {ICB }}$ the heat flow from the inner core, $Q_{\mathrm{C}}$ the secular cooling term associated with the heat capacity, $Q_{\mathrm{H}}$ the radiogenic heating term in heat producing elements are contained in the core, $Q_{\mathrm{L}}$ the latent heat produced by core freezing, and $E_{\xi}$ the compositional energy arising from redistribution of solute in a spatially varying chemical potential. The full expressions for these terms, which have been derived from a number of papers (e.g., Buffett et al. 1992, 1996; Braginsky and Roberts 1995; Lister and Buffett 1995; Labrosse et al. 1997, 2001; Labrosse 2003; Gubbins et al. 2004; Nimmo et al. 2004; Nimmo 2007), will only be briefly explained.

The secular cooling term always exists and represents the change of internal energy of the core with time. Using temperature as thermodynamic variable (instead of entropy which appears as a simpler choice when dealing with the average state of the core), one writes the secular cooling term as

$$
Q_{\mathrm{C}}=-\int_{\nu} \rho C_{\mathrm{p}} \frac{\partial T}{\partial t} \mathrm{~d} V .
$$

The time derivative introduced here must be understood as a derivative with respect to the long term evolution. Indeed, the evolution of planetary cores involves at least two very different timescales. The first one is short and is related to the dynamics of the dynamo and the second one is related to the long term evolution. These two scales are vastly different and their separation was explained in detail by Braginsky and Roberts (1995). When dealing with the long term evolution, as is done here, the dynamical processes are averaged out and only considered as ways of maintaining the core close to its reference state. It is usually assumed that vigorous convection maintains the core close to isentropic on average. In this case, the isentropic temperature profile (see (7)) can be used to compute $Q_{\mathrm{C}}$.

The isentrope is the solution of a differential equation and depends on the boundary condition, which is the only part varying with time in the isentrope. If the core is entirely liquid, the temperature at either the center or the CMB can serve as a boundary condition. In standard evolution scenarios, however, the inner core starts to crystallize at the center (Jacobs 1953) and it appears more natural to use the value at the center, $T_{\mathrm{C}}$ as the timevarying parameter. In this case, the secular cooling term takes the form

$$
Q_{\mathrm{eC}}=-P_{\mathrm{eC}} \frac{\mathrm{d} T_{\mathrm{c}}}{\mathrm{d} t}
$$

with $P_{\mathrm{eC}}$ a parameter having the units of $J \mathrm{~K}^{-1}$ which can be computed from the isentropic profile and the density profile. When an inner core has started to crystallize, a natural boundary condition appears: the temperature at the phase boundary is equal to the liquidus of the outer core. This temperature also varies with time for two reasons. First, as the inner core grows, the pressure of its upper boundary (commonly called the inner core boundary, ICB) decreases, leading to a decrease of the liquidus temperature in the Earth's core. (In smaller planets, the liquidus temperature may increase with pressure; see below.) The other reason is linked to the time evolution of the core composition. The other reason is linked to the time evolution of the core composition. Due to the partitioning of light elements in the outer core during fractional crystallization at the ICB, the liquidus 
temperature decreases with time (Stevenson et al. 1983; Lister 2003; Gubbins et al. 2004; Nimmo 2007). Both variations are linked to the growth of the inner core and the ICB boundary temperature may be replaced with the radius of the inner core $r_{\mathrm{ic}}$. For this reason, when an inner core is present and growing, the secular cooling term of the energy equation can be written as

$$
Q_{\mathrm{lC}}=P_{\mathrm{lC}}(c) \frac{\mathrm{d} c}{\mathrm{~d} t},
$$

where $P_{\mathrm{IC}}\left(r_{\mathrm{ic}}\right)$ is a function of the inner core radius $r_{\mathrm{ic}}$ and has the unit of $J \mathrm{~m}^{-1}$. The volume $V$ of integration is the total volume of the core, including the inner core, which explains the absence of an explicit heat flow from the inner core. Strictly speaking, this heat flow should be computed by solving a diffusion equation for the inner core (Labrosse et al. 1997) but owing to the small size of the inner core, this term can be approximated by extending the isentrope into the inner core.

The case of the latent heat of freezing is straightforward and the rate of energy release by this process is directly proportional to the growth rate of the inner core. It simply reads as

$$
Q_{\mathrm{L}}=T_{\mathrm{L}}(c) \rho \Delta S 4 \pi c^{2} \frac{\mathrm{d} c}{\mathrm{~d} t} \equiv P_{\mathrm{L}}(c) \frac{\mathrm{d} c}{\mathrm{~d} t},
$$

with $\Delta S$ the entropy of freezing.

The compositional energy is related to the secular change of composition of the outer core in which a gradient of chemical potential is maintained by gravity and reads (Lister and Buffett 1995; Braginsky and Roberts 1995)

$$
E_{\xi}=\int_{\mathrm{OC}}\left(\mu-\mu_{\mathrm{ICB}}\right) \frac{\mathrm{d} \xi}{\mathrm{d} t} \mathrm{~d} V
$$

with $\mu$ the chemical potential, and $\xi$ the mass fraction of light elements. The volume of integration is the outer core volume only. Since the core is assumed to be well mixed at all times, $\mathrm{d} \xi / \mathrm{d} t$ is also uniform and $E_{\xi}$ is found to be proportional to it. The profile of chemical potential is prescribed in the reference state in much the same way as that of the temperature. The change of composition is due to fractionation upon inner core freezing and is therefore proportional to the rate of inner core growth and this term, as well as the previous ones, can be written as

$$
E_{\xi}=P_{\xi}(c) \frac{\mathrm{d} c}{\mathrm{~d} t}
$$

Finally, the radiogenic heating is independent of the inner core size, and simply reads

$$
Q_{\mathrm{H}}(t)=M_{\mathrm{C}} \sum_{i=1}^{N} h_{i} \mathrm{e}^{-\lambda_{i} t}
$$

summing the $N$ contributions of all radioactive isotopes assumed to be present in the core, each one of which releases heat at a rate $h_{i}$ per unit mass at time $t=0$ and having a decay constant $\lambda_{i}$. The mass considered here, $M_{\mathrm{C}}$, is the total mass of the core, which implies that any chemical exchange with the mantle is neglected. The partitioning of radioactive elements between the outer and the inner core is of no consequence in this calculation.

The energy balance does not include any contribution from the magnetic field, thus it cannot be used to discuss dynamo generation. This comes from the smallness of the magnetic energy (Gubbins 1977) and a well-known result of the thermodynamics of convective 
flows (Hewitt et al. 1975) that dissipation is internally balanced by the work of buoyancy forces. For this reason, Aubert et al. (2009) define a Rayleigh number for the dynamo based on the total dissipation, which can be obtained using the entropy balance of the core:

$$
\frac{Q_{\mathrm{CMB}}}{T_{\mathrm{CMB}}}=\frac{Q_{\mathrm{ICB}}+Q_{\mathrm{L}}}{T_{\mathrm{ICB}}}+\frac{Q_{\mathrm{H}}}{T_{\mathrm{H}}}+\frac{Q_{\mathrm{C}}}{T_{\mathrm{C}}}+\frac{Q_{\mathrm{D}}}{T_{\mathrm{D}}},
$$

where each of the heat sources from the energy balance equation (8) enters divided by the effective temperature at which it is provided and where $Q_{\mathrm{D}} / T_{\mathrm{D}}$ is the total internal dissipation:

$$
\frac{Q_{\mathrm{D}}}{T_{\mathrm{D}}}=\int_{\mathrm{OC}}\left[k\left(\frac{\nabla T}{T}\right)^{2}+\frac{\boldsymbol{\tau}: \nabla \mathbf{u}}{T}\right] \mathrm{d} V+\int_{V^{\infty}} \frac{\mathbf{J}^{2}}{\sigma T} \mathrm{~d} V
$$

with $J$ the electrical current density and $\sigma$ the electrical conductivity. The ohmic dissipation term in (17) is integrated over all space because the magnetic field produced inside the core spreads out to the universe and its time variation induces currents in all conductors, which produces entropy. The energy required to maintain this dissipation is transported from the core along the Poynting vector. In the case of the Earth, the mantle is usually assumed to be insulating, which is equivalent to neglecting its contribution to the ohmic heating. The inner core is likely as conducting as the outer core but, in the case of the Earth again, its volume is small and its contributions to the balance equations can be approximated reasonably well by assuming its temperature profile to be isentropic (Labrosse et al. 2001). In this case, the energy and entropy balances are simplified by extending over the entire core (Labrosse 2003; Lister 2003). For other planets such as Mercury, the situation can be more complex, with a larger inner core (Stanley et al. 2005) or with a thick stagnant layer at the top of the core (Christensen 2006).

The equations for the energy and entropy balances can be combined to eliminate the total heat loss $\left(Q_{\mathrm{CMB}}\right)$ and arrive at an efficiency equation that relates the total dissipation to all energy sources:

$$
\begin{aligned}
Q_{\mathrm{D}}= & \frac{T_{\mathrm{D}}}{T_{\mathrm{CMB}}}\left(1-\frac{T_{\mathrm{CMB}}}{T_{\mathrm{C}}}\right) Q_{\mathrm{C}}+\frac{T_{\mathrm{D}}}{T_{\mathrm{CMB}}}\left(1-\frac{T_{\mathrm{CMB}}}{T_{\mathrm{ICB}}}\right)\left(Q_{\mathrm{L}}+Q_{\mathrm{ICB}}\right) \\
& +\frac{T_{\mathrm{D}}}{T_{\mathrm{CMB}}}\left(1-\frac{T_{\mathrm{CMB}}}{T_{\mathrm{H}}}\right) Q_{\mathrm{H}}+\frac{T_{\mathrm{D}}}{T_{\mathrm{CMB}}} E_{\xi} .
\end{aligned}
$$

The value of $T_{\mathrm{D}}$ is difficult to estimate but since the CMB is likely the coldest place of the core, the ratio $T_{\mathrm{D}} / T_{\mathrm{CMB}}>1$. Therefore, the efficiency factor for converting heat into work is larger than that of a Carnot engine (Hewitt et al. 1975). This comes from the fact that - contrary to Carnot engines-work is performed inside the convective region and the dissipative heating is not lost but can contribute to fuel motion. Equation (18) also shows that the conversion of compositional energy is not affected by the Carnot factor and has a larger efficiency than heat sources.

All the terms on the right-hand side of (18) can be computed from the reference state of the core in much the same way as the energy terms, except for the factor $T_{\mathrm{D}}$. Each term, except for the radiogenic heat source term, can be linked to the inner core and is proportional to its growth rate. The total dissipation $Q_{\mathrm{D}} / T_{\mathrm{D}}$ contains three contributions. The contribution from thermal diffusion (chemical diffusion should also be included but is generally neglected with good reason), $\int k(\nabla T / T)^{2} \mathrm{~d} V$ is easily computed from the basic isentropic state. The remaining two contributions are associated with the work of buoyancy forces 
and contain a contribution from viscous friction, which is usually neglected (see Braginsky and Roberts 1995, for a justification), and a contribution from ohmic dissipation which is relevant to the dynamo problem:

$$
\Phi=T_{\mathrm{D}} \int \frac{J^{2}}{\sigma T} \mathrm{~d} V
$$

with $\mathbf{J}=\nabla \times \mathbf{B} / \mu_{0}$ the electrical current density and $\sigma$ the electrical conductivity. The relationship of $J$ to $B$ involves a spatial derivative so that any estimate of the former from the latter is plagued by large uncertainties at small length-scales. Also, $B$ contains both a poloidal and a torroidal component. The torroidal component is confined to the planetary cores and cannot be measured directly. Therefore, the ohmic dissipation rate is badly constrained for the Earth and even more so for all other planets.

\subsection{Snow and Exsolution}

The energy and entropy balances discussed in the preceding sections are based on the assumption of an isentropic reference state in the core. The main argument supporting this assumption is that, if the dynamics is sustained by both thermal and compositional destabilizing gradients, convection will tend to homogenize the extensive state variables responsible for the motion, entropy, and composition. Intensive variables like temperature and chemical potential can obviously not be homogenized and their gradients establish a disequilibrium that maintains motion. The assumption of widely uniform composition and entropy is consistent with a very large Rayleigh number (values of $10^{29}$ and more are considered) and the smallness of the lateral variations of temperature at the top of the Earth's core supports this assumption.

Recent experimental work (Fei et al. 1997, 2000; Chudinovskikh and Boehler 2007) have identified two important aspects of the Fe-FeS phase diagram that apply to the smaller Earth-like planets and satellites (e.g., Mars, Moon, Mercury, the Galilean satellites, and Titan): (1) at pressures lower than $14 \mathrm{GPa}$ the eutectic melting temperature decreases with increasing pressure, and (2) at pressures lower than $40 \mathrm{GPa}$ the eutectic sulfur concentration decreases with increasing pressure.

A melting temperature with a negative slope will have profound implications. Other than at the elevated pressure of the Earth's core where the eutectic melting temperature increases with pressure, Fe may precipitate at the $\mathrm{CMB}$ rather than in the center and may fall as iron snow (Fig. 9 below shows a schematic representation of the Fe-FeS melting diagram and compares a snow model for Ganymede with a convectional inner core growth model). Note, however, that experimental data are only available to date for pure iron and eutectic $\mathrm{Fe}-\mathrm{FeS}$. Models require interpolations between these two curves that must remain speculative to some extent. For instance, it is not certain at which composition the positive slope of the Fe-rich liquidus will turn into a negative one. Bland et al. (2008) argue that the transition may occur at 3 weight $\%$ of sulfur.

Two scenarios for a Fe-snowing core can be envisioned depending on whether the core is in thermal equilibrium or not: In equilibrium (or close to equilibrium), the sinking iron will initially remelt at greater depths. As a consequence, the concentration of Fe will increase with depth in the precipitation zone, a stable density gradient will form, and the liquidus and core temperature profile will become co-linear. The layer below the precipitation zone will be well mixed and will become increasingly iron rich with time. With further cooling, the precipitation zone will grow in thickness. When it extends through the entire 
core, a solid inner core will form upon further cooling. In the second scenario, rapid crystal sinking-possibly supported by convective overturn induced by lateral differences in crystal concentration - and slow melting kinetics may prevent equilibrium (Stewart et al. 2007). A metastable agglomerate of iron will then form a solid iron inner core.

The iron snow model has been applied to Mars (Stewart et al. 2007), Mercury (Chen et al. 2008), and Ganymede (Hauck et al. 2006; Bland et al. 2008). Whether or not iron snow can drive a dynamo in these planets is still unclear, however, and controversially discussed. No magneto-hydrodynamic dynamo models driven by iron snow have been published yet.

A further variant of the snowing core model has been discussed in particular for Ganymede. If the composition of the core were sulfur-rich, iron sulfide (FeS) would precipitate instead of Fe. Solid FeS has a density lower than that of liquid Fe-FeS in the pressure range of Ganymede's core (Fei et al. 1995; Balog et al. 2003) and would float upward from the deep core to form a solid FeS layer on top of the core (Hauck et al. 2006). Hauck et al. (2006) speculate that this process may also drive a dynamo but the model has not been studied in much detail.

Another possibility for compositional convection introduced by Stevenson (1983) and presented at several conferences thereafter is related to exsolution of light elements such as $\mathrm{Si}, \mathrm{O}, \mathrm{S}, \mathrm{Mg}$ upon cooling of the core. The solubility of light elements in iron generally decreases with temperature. Considering the large amount of cooling of the core since the formation of the Earth (as discussed in Sect. 5.1), it is conceivable that light elements were exsolved if the core started not too far from saturation. On the other hand, the pressure of equilibration between core and mantle materials is between 20 and $40 \mathrm{GPa}$ (e.g., Thibault and Walter 1995; Li and Agee 1996), much lower than the pressure in the core. It is thus possible that during differentiation liquid iron blobs incorporated light elements at low pressures that would later not be in equilibrium at the high pressures of the core. Experimental studies of core-mantle chemical interaction (Knittle and Jeanloz 1991; Goarant et al. 1992; Ozawa et al. 2008), however, tend to show that the core could readily dissolve a larger amount of light elements than is consistent with its observed density. The exsolution scenario, therefore, seems unlikely to work for the Earth, at least if the most commonly discussed ones ( $\mathrm{Si}, \mathrm{O}, \mathrm{S}$ ) are considered. However, should light elements reach saturation (Stevenson mentions $\mathrm{Mg}$ ), the density difference involved would be large and could drive strong flows and possibly a dynamo.

\section{The Mantle Reigns the Core}

\subsection{Mantle Convection}

In the foregoing section, we have discussed how dynamo action depends on the cooling of the core. Since the core heat must flow through the mantle, it is heat transfer through the mantle that regulates the cooling of the core. A discussion of magnetic field generation in planetary cores must, therefore, include a discussion of mantle heat transfer and the thermal evolution of the planet. It is widely agreed that mantle heat transfer is mostly by convection and heat conduction. To model heat transfer by convection two methods are mostly used: the first is based on numerical solutions of the hydrodynamic field equations. The second uses semiempirical relations between the heat transfer rate and the vigor of mantle convection. 


\subsubsection{Finite Amplitude Convection Models}

With finite amplitude convection models in 2D or 3D geometry, sets of nonlinear differential equations are solved that include conservation of mass, energy, and momentum balance equations and an equation of state subject to suitable boundary conditions. These models yield velocity and temperature fields as functions of time, and thus provide details of the mantle flow and the associated heat transport. In most convection models, the mantle is considered as an incompressible and highly viscous fluid for which inertia forces in the momentum equation can be neglected. Moreover, the Boussinesq approximation is most often taken, for which the density is assumed constant except for in the buoyancy term. An extended version of the Boussinesq approximation includes the effects of viscous heating as well as adiabatic cooling and heating (see Schubert et al. 2001). (Note that the term adiabatic is conventionally used in the convection literature instead of isentropic. An adiabatic state is equivalent to the isentropic state in the absence of contributions to entropy other than from heat flow across the boundaries.) The mantle may be compositionally layered or mantle layers may be separated by phase transition boundaries. Some models (e.g., Stegman et al. 2003a, 2003b) consider gradients in composition and include mass transfer equations to model thermo-chemical convection. In the following, we present the non-dimensional equations for Boussinesq thermal convection models (compositional variations are not considered).

The conservation of mass, momentum, and energy equations read

$$
\begin{gathered}
\nabla \cdot \mathbf{u}^{\prime}=0, \\
\nabla p^{\prime}=\nabla \cdot\left(\eta^{\prime}\left(\nabla \mathbf{u}^{\prime}+\left\{\nabla \mathbf{u}^{\prime}\right\}^{\mathrm{T}}\right)\right)+R a T^{\prime} \mathbf{e}_{\mathrm{r}}, \\
\frac{\partial T^{\prime}}{\partial t^{\prime}}+\mathbf{u}^{\prime} \nabla T^{\prime}=\nabla^{2} T^{\prime}+\frac{R a_{\mathrm{H}}}{R a},
\end{gathered}
$$

where all primed quantities are nondimensionalized and scaled, $\nabla$ denotes the nabla operator, $\eta^{\prime}$ the viscosity, $\mathbf{u}^{\prime}$ the velocity vector, $p^{\prime}$ the dynamic pressure, \{\}$^{\mathrm{T}}$ the tensor transpose, $T^{\prime}$ the temperature, $t^{\prime}$ the time, and $\mathbf{e}_{\mathrm{r}}$ the unit vector in radial direction. The Rayleigh number measures the buoyancy term relative to the retarding effects of viscosity and buoyancy loss by heat conduction, and thus the strength of the convection. For a fluid heated from below, it is defined as

$$
R a=\frac{\alpha \rho_{\mathrm{m}} g \Delta T d^{3}}{\kappa \eta_{\mathrm{ref}}}
$$

and for a fluid heated from within as

$$
R a_{\mathrm{H}}=\frac{\alpha \rho_{\mathrm{m}} g Q_{\mathrm{m}} d^{5}}{k \kappa \eta_{\mathrm{ref}}}
$$

with $\rho_{\mathrm{m}}$ the mantle density, $\kappa$ the thermal diffusivity, $d=r_{\mathrm{p}}-r_{\mathrm{c}}$ the mantle or layer thickness, $r_{\mathrm{p}}$ the planetary radius, $r_{\mathrm{c}}$ the core radius, $\Delta T$ the super-adiabatic temperature difference across the convecting mantle, $\eta_{\text {ref }}$ the reference viscosity, $Q_{\mathrm{m}}$ the volumetric heating rate in the mantle (heat produced by the decay of radioactive elements), and $k$ the thermal conductivity. The ratio of $R a_{\mathrm{H}} / R a$ is a dimensionless measure of the amount of internal heating. 
Typical boundary conditions are free-slip mechanical boundary conditions at the coremantle interface (CMB) and no slip or free-slip conditions at the surface. For the temperature boundary conditions, a constant temperature is usually assumed at the surface and a constant temperature or a constant heat flow at the CMB.

The viscosity is the most important parameter for understanding the role of mantle convection in transporting heat. The temperature dependence of the viscosity acts as a thermostat to regulate the mantle temperature. In addition to the temperature dependence, the rheology in a planetary mantle can be described by two main creep mechanisms: diffusion creep and dislocation creep. For the case of diffusion creep, the solid behaves as a Newtonian fluid where the viscosity is independent of the applied shear stresses. In contrast, for dislocation creep, the solid behaves as a non-Newtonian fluid where viscosity varies with the applied shear stress. Indeed, viscosity tends to decrease with increasing shear stress, often nonlinearly. It is not certain which creep mechanism is valid in terrestrial mantles. Most laboratory studies of mantle deformation have concluded that dislocation creep is the applicable deformation mechanism in the upper Earth mantle and diffusion creep in the lower mantle (Schubert et al. 2001). However, this observation is not consistent with post-glacial rebound studies that favor diffusion creep also for the upper mantle. Moreover, laboratory experiments have suggested that the pressure-dependence of viscosity cannot be neglected for a terrestrial mantle (Karato and Rubie 1997). Thus, the viscosity of a terrestrial mantle can be described with the following Arrhenius relationship:

$$
\eta=\frac{\mu^{n}}{2 A}\left(\frac{1}{\tau}\right)^{n-1}\left(\frac{h}{B^{*}}\right)^{m} \exp \left(\frac{E+p V}{R T}\right)
$$

with $\mu$ the shear moduls ( $\sim 80 \mathrm{GPa}) . A$ is a preexponential factor, $\tau$ the shear stress, $h$ the average grain size, $m$ the grain size exponent, $B^{*}$ the length of the Burgers vector $(\sim 0.5 \mathrm{~nm})$, $E$ the activation energy, $p$ the pressure, $V$ the activation pressure, and $R$ the gas constant. For a Newtonian rheology, $n$ is equal to 1 and for a non-Newtonian rheology a typical value of $n$ is 3.5. For most numerical studies, however, an exponential viscosity law, termed the Frank-Kamenetskii approximation, is used.

$$
\eta=\frac{f}{\tau^{n-1}}\left(\exp \left(-\gamma T^{\prime}\right)+\ln \left(\Delta \eta_{\mathrm{p}}\right)\left(r_{\mathrm{p}}^{\prime}-r^{\prime}\right)\right)
$$

where $f$ is a constant, $\gamma$ is related to (25) through $\gamma=E / R T_{i}^{\prime 2}$ for purely temperaturedependent viscosity, and $\Delta \eta_{\mathrm{p}}$ is the viscosity increase due to pressure.

Convection Regimes The convection pattern and the heat transfer rate through a planetary mantle vary significantly with the vigor of convection measured by the Rayleigh number and the viscosity variation $\Delta \eta=\exp (\gamma)$ across the mantle. Depending on the viscosity contrast, convection may reach to the top (and cold) surface or a stagnant lid may form underneath that surface. Four different regimes have been identified (e.g., Hansen and Yuen 1993; Solomatov 1995; Trompert and Hansen 1998; Huettig 2009) (Fig. 1):

The mobile regime is typical for convection in an isoviscous fluid or for convection in a fluid with sufficiently low viscosity contrast. It is characterized by the flow reaching all the way up to the surface. The surface is said to be fully mobilized in this regime.

The sluggish regime, sometimes called transitional regime, is typical for convection in fluids with moderate viscosity variation and for dominantly bottom heated convection in plane layers. The surface is almost stagnant here due to the high viscosity of the fluid near the cold surface. 

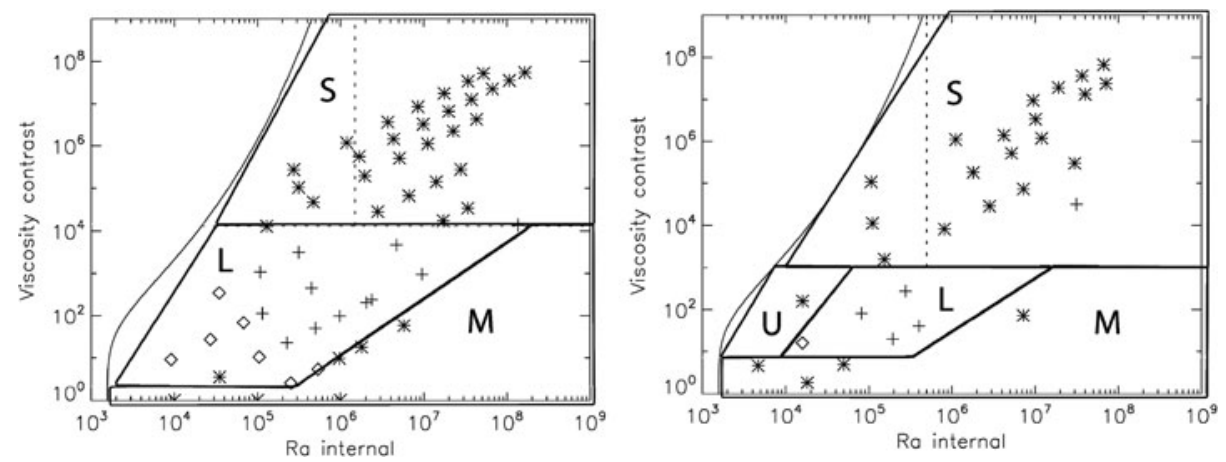

Fig. 1 Convection platform regimes are shown in the $R a_{\mathrm{H}}$ (see (24)) and $\Delta \eta$ parameter plane for internally heated spherical shells with temperature dependent viscosity (left) and temperature and pressure dependent viscosity (right). (The pressure increases linearly thorough the layer and the variation of the viscosity due to pressure variation is a factor of 100.) $M$ denotes the mobile regime, $U$ the sluggish regime, $L$ the low-degree regime, and the $S$ the stagnant-lid regime. Crosses mark degree-one, diamonds degree-two, and stars higher degrees of convection. The vertical dashed line divides the stagnant lid regime further into a steady regime (to the left of the line) and a time dependent regime (to the right of the line) (From Huettig 2009)

Table 2 Flow law parameters for olivine (Karato and $\mathrm{Wu}$ 1993). Dry refers to water-free and wet to water-saturated conditions, respectively

\begin{tabular}{llllll}
\hline & \multicolumn{2}{l}{ Dislocation creep } & & \multicolumn{2}{l}{ Diffusion creep } \\
\cline { 2 - 3 } \cline { 5 - 6 } \cline { 5 - 6 } & Dry & Wet & & Dry & Wet \\
\hline$A\left[\mathrm{~s}^{-1}\right]$ & $3.5 \times 10^{22}$ & $2.0 \times 10^{18}$ & & $8.7 \times 10^{15}$ & $5.3 \times 10^{15}$ \\
$N$ & 3.5 & 3.0 & & 1.0 & 1.0 \\
$M$ & 0 & 0 & & 2.5 & 2.5 \\
$E\left[\mathrm{~kJ} \mathrm{~mol}^{-1}\right]$ & 540 & 430 & & 300 & 240 \\
$V\left[\mathrm{~cm}^{3} \mathrm{~mol}^{-1}\right]$ & $10-25$ & $10-20$ & & 6 & 5 \\
$h[\mathrm{~m}]$ & - & - & & $10^{-2}-1$ & $10^{-2}-1$ \\
\hline & & & &
\end{tabular}

The low-degree regime is typical of convection in a fluid mostly heated from within and in spherical shells. This regime may entirely replace the sluggish regime in fluids with no or pressure dependence of viscosity or may replace it at high Rayleigh numbers for pressure dependent viscosity. The regime is characterized by long wavelengths flow patterns.

The stagnant-lid regime occurs for strongly temperature dependent viscosity fluids. The near surface fluid here is completely stagnant and does not participate in the convection. It forms a lid and most of the viscosity variation occurs in the lid. The fluid underneath the lid is almost isoviscous. The thickness of the lid increases with increasing viscosity contrast but is also a function of the heat flow through the entire layer. Since the lid is stagnant, heat transfer through the lid is by thermal conduction.

Applying the parameter values assumed characteristic of the rheology of mantle rock (Table 2), the viscosity contrast across the mantles of the terrestrial planets is several orders of magnitude. Thus, all terrestrial planetary bodies even the Earth should fall into the stagnant lid regime. The existence of plate tectonics on Earth cannot be understood from the temperature dependence of mantle viscosity alone and requires the consideration of additional factors such as strain localization and weakening to form plate boundaries. For the Earth, this is achieved by faulting in the brittle and elastic upper part of the lithosphere and by ductile shear localization below this layer. The latter is thought to be the most important 
mechanism for generating weak plate boundaries and occurs through grain size reduction, void-volatile weakening or viscous dissipation (for a review, see Tackley 2000a). Two simplified rheology models-strain rate weakening (e.g., Bercovici 1998) and plastic yielding (e.g., Tackley 2000b) — have been used to model plate tectonics. In strain rate weakening, stress initially increases with increasing strain rate and reaches a maximum at some critical rate. In plastic yielding, the viscosity is rapidly decreased beyond a transition stress. Although substantial improvements in the modeling of plate tectonics have been made in recent years, convection models with self-consistent plate generation are still in their infancy. The models are, for instance, not capable of simulating one-sided subduction, i.e., the subduction of one plate underneath another, a common feature on Earth and do not use realistic values of the yield strength.

Thermal Evolution Models with 2D or 3D Convection Models Numerical solutions of (20) to (22) are often used to study the steady state or quasi-steady state of mantle flow. The typical model runs then determine, e.g., the flow structure, the heat flow (Nusselt number), the boundary layer thicknesses, and the temperature distribution. Steady state models, however, cannot be directly used to infer the thermal evolution of terrestrial planets. Instead they are useful to explore the various convection regimes and derive appropriate scaling laws. These can be used to parameterize the convective heat transfer rate as discussed in Sect. 4.1.2 below.

Other numerical solutions include time dependence to model the thermal evolution of terrestrial planets (e.g., Steinbach and Yuen 1994; Konrad and Spohn 1997; Conzelmann 1999; Spohn et al. 2001c; Buske 2006; Nakagawa and Tackley 2004; Xie and Tackley 2004; Butler et al. 2005; Costin and Butler 2006; Ziethe et al. 2009; Keller and Tackley 2009). These models require some modifications with respect to steady-state models in the parameters and the boundary conditions, in particular, if the core is to be included. The internal heating rate $Q_{\mathrm{m}}$ cannot be taken constant but should decrease exponentially with time $\left(Q_{\mathrm{m}}=Q_{0} \exp \left(-\sigma_{\mathrm{r}} t\right)\right)$ with the average decay rate of the radioactive elements $\sigma_{\mathrm{r}}$ and the initial value $Q_{0}$. Furthermore, the temperature at the CMB is not constant but decreases according to how much heat is transported out of the core by the mantle. To obtain the coremantle boundary temperature, an energy equation for the core needs to be solved (compare also (29) below). The time rate of change of internal energy of the core is equated to the heat flow out of the core. If inner growth is neglected, the following dimensionless equation results

$$
\frac{\mathrm{d} T_{\mathrm{cmb}}^{\prime}}{\mathrm{d} t^{\prime}}=\frac{3}{\varepsilon_{\mathrm{c}} r_{\mathrm{c}}^{\prime}} \frac{\rho_{\mathrm{m}} C_{\mathrm{m}}}{\rho_{\mathrm{c}} C_{\mathrm{c}}} \frac{\mathrm{d} T_{\mathrm{cmb}}^{\prime}}{\mathrm{d} r^{\prime}},
$$

where $T_{\mathrm{cmb}}^{\prime}$ is the nondimensional core-mantle boundary temperature, $C_{\mathrm{m}}$ and $C_{\mathrm{c}}$ the mantle and core heat capacities, respectively, $r_{\mathrm{c}}^{\prime}$ the nondimensional core radius, and $\varepsilon_{\mathrm{c}}$ is the ratio between the core temperature that is representative of the internal energy of the core and $T_{\mathrm{cmb}}$.

\subsubsection{Parameterized Convection Models}

Most thermal evolution models of terrestrial planets that have been calculated to date use the approach of parameterized convection, however. These models use parameterizations of the convective heat transfer rate derived from finite amplitude convection models or from laboratory experiments (see Schubert et al. 2001 for a discussion). The method is restricted to the calculation of global properties such as the mean mantle and core temperature, the 


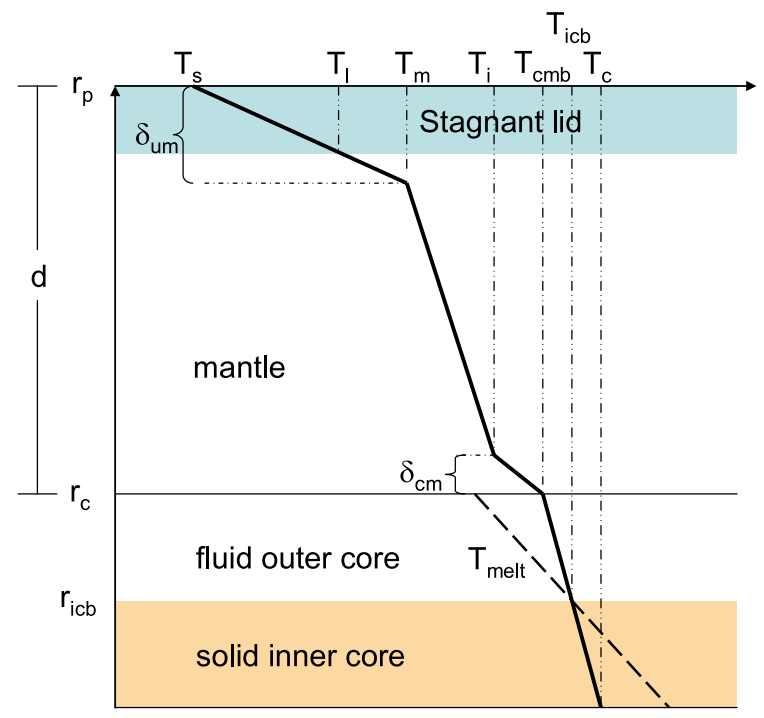

Fig. 2 Thermal structure of the interior of a planet or satellite with notations used in the text. The bluish region is the stagnant lid, the bold solid line marks the temperature profile, the long dashed line marks the melting temperature in the core, and the orange region is the solid inner core. Dash-dotted lines indicate specific temperature and layer thicknesses. $T_{\mathrm{S}}$ is the surface temperature. $T_{1}$ is the temperature at the base of the stagnant lid and $T_{\mathrm{m}}$ is the temperature at the base of the upper thermal boundary layer. $T_{\mathrm{i}}$ is the temperature at the top of the lower thermal boundary layer and $T_{\mathrm{cmb}}$ is the temperature at the core-mantle boundary which coincides with the base of the lower thermal boundary layer of mantle convection. $T_{\mathrm{icb}}$ is the temperature at the top of the inner core and $T_{\mathrm{c}}$ is the central temperature. $\delta_{\mathrm{um}}$ is the thickness of the upper and $\delta_{\mathrm{cm}}$ that of the lower thermal boundary layer of mantle convection. $r_{\mathrm{p}}, r_{\mathrm{c}}$, and $r_{\mathrm{icb}}$ are the radii of the surface, the core, and the inner core, respectively, and $d$ denotes the thickness of the mantle

average surface and core-mantle heat flow, the lithosphere thickness and the mean mantle velocity as functions of time only. These models have the advantage of not being limited by available computer power and extensive parameter searches can be performed.

In the following, we briefly present the basic equations and methods used for parameterized convection models. In the next section, we will discuss applicable scaling laws. Detailed discussions of the equations and the methods used to derive them can be found in the literature cited below. Some models include the growth of a crust by mantle partial melting and differentiation and the associated redistribution of radioactive elements (e.g., Hauck et al. 2002, 2004; Breuer and Spohn 2003; Schumacher and Breuer 2006; Grott and Breuer 2008). These, however, are not considered in the present work.

The basic equations setting up a thermal evolution model are energy balance equations for the mantle and the core (see also Fig. 2 for a definition of relevant variables and parameters). The energy equation for the mantle is

$$
\rho_{\mathrm{m}} C_{\mathrm{m}} V_{\mathrm{m}} \varepsilon_{\mathrm{m}} \frac{\mathrm{d} T_{\mathrm{m}}}{\mathrm{d} t}=-q_{\mathrm{m}} A_{\mathrm{m}}+Q_{\mathrm{m}} V_{\mathrm{m}}
$$

with $V_{\mathrm{m}}$ and $A_{\mathrm{m}}$ the volume and the surface of the mantle, respectively. $T_{\mathrm{m}}$ is the mantle temperature at the bottom of the thermal boundary layer, $\varepsilon_{\mathrm{m}}$ is the ratio between the mantle temperature that is representative of the internal energy of the mantle and $T_{\mathrm{m}}$ and $q_{\mathrm{m}}$ is the heat flow out of the mantle. 
The energy equation of the core is

$$
\rho_{\mathrm{c}} C_{\mathrm{c}} V_{\mathrm{c}} \varepsilon_{\mathrm{c}} \frac{\mathrm{d} T_{\mathrm{cm}}}{\mathrm{d} t}=-q_{\mathrm{c}} A_{\mathrm{c}}+\left(L+E_{\mathrm{g}}\right) \frac{\mathrm{d} m_{\mathrm{ic}}}{\mathrm{d} t}
$$

with $V_{\mathrm{c}}$ and $A_{\mathrm{c}}$ the volume and the surface of the core, respectively. $T_{\mathrm{cm}}$ is the temperature at the core-mantle boundary, $\varepsilon_{\mathrm{c}}$ is the ratio between the core temperature that is representative of the internal energy of the core and $T_{\mathrm{cm}}$ and $q_{\mathrm{c}}$ is the heat flow out of the core. The second term on the right side describes the energy released upon growth of an inner core of mass $m_{\mathrm{ic}}$, with the latent heat $L$ and the gravitational energy $E_{\mathrm{G}}$ that is released if the inner core composition is denser than the outer core. To solve for $\mathrm{d} m_{\mathrm{ic}} / \mathrm{d} t$, the melting temperature and the adiabat of the core as a function of pressure are required. The radius of the inner core is at a melting temperature (compare Fig. 2 and Breuer et al. 2007).

The heat flow out of the convecting mantle can be calculated from

$$
q_{\mathrm{m}}=N u \frac{k \Delta T}{d}=k \frac{\Delta T_{\mathrm{sm}}}{\delta_{\mathrm{um}}}
$$

with $N u$ the Nusselt number, i.e., the dimensionless heat flux out of the convecting layer, $\delta_{\mathrm{um}}$ the thicknesses of the upper thermal boundary layer (including the stagnant lid), $\Delta T=$ $\Delta T_{\mathrm{sm}}+\Delta T_{\mathrm{cm}}$ with $\Delta T_{\mathrm{sm}}=T_{\mathrm{m}}-T_{\mathrm{s}}$ the temperature difference across the upper thermal boundary, $\Delta T_{\mathrm{cm}}=T_{\mathrm{cm}}-T_{\mathrm{i}}$ the temperature difference through the lower thermal boundary layer, $T_{\mathrm{s}}$ the surface temperature, and $T_{\mathrm{i}}$ the temperature at the top of the lower thermal boundary layer (Fig. 2).

The temperature $T_{\mathrm{i}}$ is given by the adiabatic temperature increase through the mantle with $T_{\mathrm{i}}=T_{\mathrm{m}}+\Delta T_{\mathrm{ad}}=T_{\mathrm{m}}+\alpha g T_{\mathrm{m}}\left(d-\delta_{\mathrm{um}}-\delta_{\mathrm{cm}}\right) / C_{\mathrm{m}}$ where $\delta_{\mathrm{cm}}$ is the thickness of the lower thermal boundary layer. The adiabatic temperature gradient can be calculated from (7).

The core-mantle heat flow $q_{\mathrm{c}}$ can be calculated from a local stability criterion (e.g., Stevenson et al. 1983; Deschamps and Sotin 2000)

$$
\begin{gathered}
q_{\mathrm{c}}=\frac{k \Delta T_{\mathrm{cm}}}{\delta_{\mathrm{cm}}}, \\
\delta_{\mathrm{cm}}=\left(\frac{\eta_{\mathrm{cm}} \kappa R a_{\delta}}{\alpha \rho g \Delta T_{\mathrm{cm}}}\right)^{1 / 3},
\end{gathered}
$$

where $\eta_{\mathrm{cm}}$ is the average viscosity of the core-mantle boundary and $R a_{\delta}$ is the lower thermal boundary layer Rayleigh number.

To solve the equations above, we need to apply scaling laws from finite amplitude models which will be discussed in the next section.

Scaling Laws for Isoviscous Convection (Plate Tectonic Planet) Thermal history models for the plate tectonics planet Earth usually apply the scaling laws derived for fluids with constant viscosity which seem to work quite well (Schubert et al. 2001). Although the viscosity in the Earth varies similarly with temperature as in Mars or Venus, this is in line with the mobile lid regime requiring small viscosity variations. Apparently, from the (limited) point of view of the theory of convection with temperature dependent viscosity, Earth behaves as if it were a constant viscosity planet; what causes the Earth to behave that way is still not completely understood. 
From classical boundary layer theory, we have (e.g., Turcotte and Oxburgh 1967; Roberts 1979)

$$
N u=a \cdot R a^{\beta},
$$

where as applied to the mantle $N u \equiv q_{\mathrm{m}} d / k \Delta T$. Equivalently, the thickness of the upper boundary layer is

$$
\delta_{\mathrm{um}}=d\left(\frac{R a_{\mathrm{cr}}}{R a}\right)^{-\beta} .
$$

The constants $a$ and $\beta$ need to be determined from either numerical finite amplitude convection calculations or experiments and depend on the boundary conditions and the heating mode. For instance, $a=0.258$ and $\beta=0.321$ for free slip boundary conditions at both the upper and lower boundary, $a=0.336$ and $\beta=0.252$ for no-slip and free slip conditions at the upper and lower boundary, respectively, and $a=0.339$ and $\beta=0.223$ for no-slip condition at both the upper and lower boundaries (Deschamps and Sotin 2000).

$R a_{\text {cr }}$ in classical boundary layer theory is the critical Rayleigh number for the onset of convection. Empirical scaling laws find a factor of proportionality that is numerically close to the critical Rayleigh number of about 500 for the onset of convection in plane layers and spherical shells (Schubert et al. 2001).

For constant viscosity and free slip boundary conditions, the critical Rayleigh number for the lower thermal boundary layer, $R a_{\delta}$, can be taken as constant with a value of 2.46 although a slight dependence on the Rayleigh number has been observed (Deschamps and Sotin 2000). Note that this value is considerably smaller than the one suggested by the experiments of Booker and Stengel (1978) of 2000 and used for instance by Stevenson et al. (1983). This is likely caused by the no-slip boundary condition in the Booker and Stengel experiments.

Scaling Laws for Strongly Temperature Dependent Viscosity (Stagnant Lid Planet) The heat transfer scaling laws used for models of the thermal evolution of one-plate (or stagnant lid) planets have evolved considerably over the past few decades. Early models applied the scaling laws (33) and (34) for fluids with constant viscosity (e.g., Sharpe and Peltier 1978, 1979; Schubert 1979; Schubert et al. 1979; Stevenson et al. 1983) but used the temperature at the base of the lithosphere (about $1100 \mathrm{~K}$ ) as surface temperature $T_{\mathrm{s}}$. Later, the models were modified to include the effects of a growing lithosphere by Schubert and Spohn (1990). Their model as well as the later models of Spohn (1991) and Schubert et al. (1992) solved the one-dimensional heat conduction equation with a Stefan-like boundary condition for the rate of growth of the lithosphere. The heat flow from the mantle was calculated using the conventional constant viscosity parameterization. The base of the lithosphere in these models is an isotherm assumed to be characteristic for the transition from viscous to rigid response to loads applied over geologic timescales. This lithosphere can be identified with a rheological lithosphere with a representative value of the isotherm of around $1100 \mathrm{~K}$.

The scaling laws for stagnant lid convection that are used in present models of the thermal evolution of one-plate planets were pioneered by Christensen (1985), Solomatov (1995), Davaille and Jaupart (1993), and Grasset and Parmentier (1998). The scaling laws differ slightly between the parameterizations but give similar results and in all cases the Nusselt number depends on the viscosity contrast in addition to its dependence on the Rayleigh number. We use the formulation of Solomatov (1995) in the following. The $N u-R a$ scaling law for stagnant lid convection is then

$$
N u=a \Theta^{-c} R a_{\mathrm{i}}^{\beta}
$$


with $R a_{\mathrm{i}}$ the Rayleigh number based on the viscosity at the base of the upper thermal boundary layer and the Frank-Kamenetskii parameter

$$
\Theta \equiv \frac{E}{R T_{\mathrm{m}}^{2}} \Delta T=\gamma \Delta T .
$$

The constants $a, c$, and $\beta$ depend on the rheology (Newtonian or non-Newtonian), the heating mode (bottom, from within, or mixed) and the geometry. For example, $a=0.67$, $b=1.33$, and $\beta=0.333$ for internal heating, Newtonian rheology and spherical geometry (Reese et al. 2005). Parameter values for a variety of models have been compiled from numerical calculations by Solomatov and Moresi (2000).

The temperature at the base of the stagnant lid $T_{1}$ is not constant as in the growing lithosphere models described above but is a function of the temperature of the underlying mantle $T_{\mathrm{m}}$, and the rate of change of viscosity with temperature:

$$
T_{\mathrm{l}}=T_{\mathrm{m}}-a_{\mathrm{rh}}\left(\frac{\mathrm{d} \ln \eta}{\mathrm{d} T}\right)^{-1}=T_{\mathrm{m}}-a_{\mathrm{rh}} \frac{R T_{\mathrm{m}}^{2}}{E}
$$

with the constant $a_{\mathrm{rh}}=\ln 10 \approx 2.21$ for a Newtonian fluid (e.g., Solomatov 1995). Equation (37) has been derived by observing that in stagnant lid convection most of the viscosity variation occurs in the lid while the convecting layer underneath supports a viscosity variation by only about a factor of 10 .

The lower thermal boundary layer critical Rayleigh number $R a_{\delta}$ has been found to depend on $R a_{\mathrm{i}}$ (Deschamps and Sotin 2000)

$$
R a_{\delta}=0.28 R a_{\mathrm{i}}^{021} .
$$

Thermal Evolution: Characteristics for Plate Tectonic and Stagnant Lid Planets If we apply the parameterized convection models described above to generic planets in either the stagnant lid or plate tectonic regime, we find characteristically different cooling behaviors (compare Fig. 3). We use parameters applicable to Mars in this comparison (see Breuer and Spohn 2006 for the chosen parameter values).

The stagnant-lid planet cools mainly from above by thickening its lithosphere (stagnant lid plus upper thermal boundary layer), to about $300 \mathrm{~km}$ in the present model. The interior remains hot - the mantle temperature increases first and decreases thereafter but after $4.5 \mathrm{Ga}$ has about the same value as the initial temperature (The isotherm moves deeper into the interior of the planet). The core-mantle boundary temperature also increases early on but after $4.5 \mathrm{Ga}$ is about $50 \mathrm{~K}$ below its starting value. The interior of a plate tectonics planet, on the other hand, cools quite efficiently. The present day mantle temperature is about $450 \mathrm{~K}$ lower than the initial temperature and the core-mantle boundary cools by $600 \mathrm{~K}$. The thermal boundary layer at the surface reaches a thickness that is only a few tens of kilometers smaller than the stagnant lid thickness. These differences in the efficiency of interior cooling have substantial consequences for the thermal evolution of the core. For the one-plate planet, the core temperature is about $550 \mathrm{~K}$ above the core temperature of the plate tectonics planet. As a consequence, core freezing and a compositionally driven dynamo will start much earlier for the plate tectonics planet. In addition, an early thermal dynamo would be active for a longer time in a planet with plate tectonics than in a planet with stagnant lid convection. In general, a planet undergoing plate tectonics is more likely to generate a magnetic field than a planet with stagnant lid convection. 


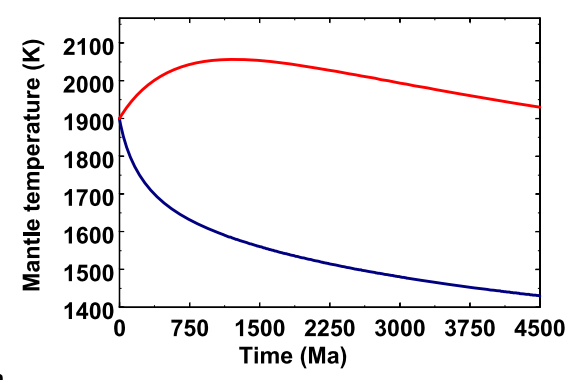

a

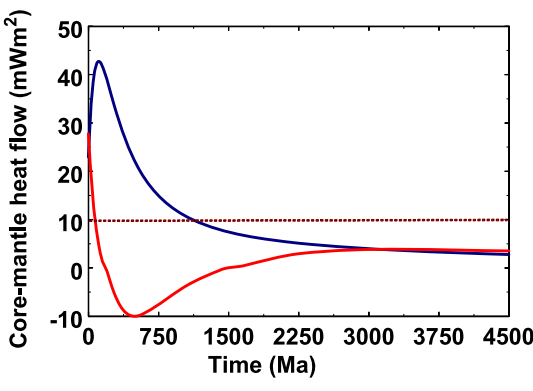

b
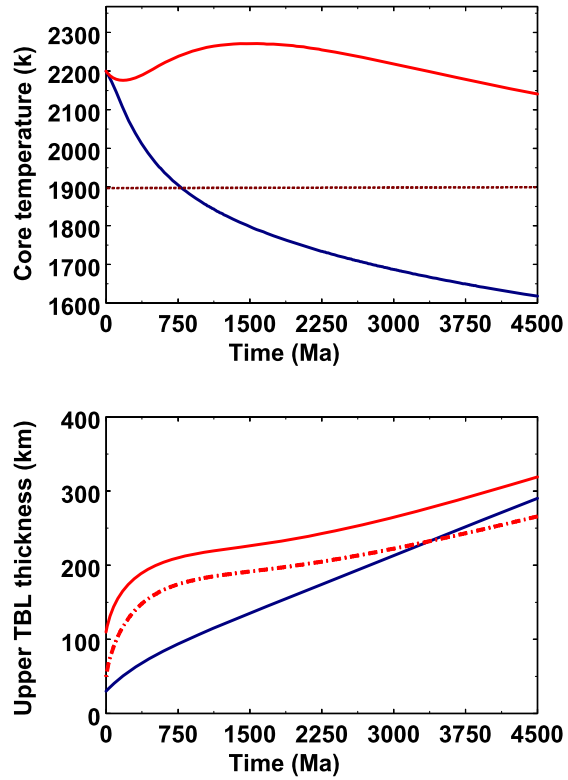

Fig. 3 Mantle temperature (a), core-mantle temperature (b), core-mantle heat flow (c), and thickness of the upper thermal boundary layer (d) are shown as functions of time for two tectonic mechanisms: stagnant lid (red line) and plate tectonics (blue line). The dashed horizontal line in panel (b) indicates the temperature at the $\mathrm{CMB}$ below which an inner core starts to grow at the center. The dashed horizontal line in panel (c) indicates the heat flow conducted along the isentrope (or adiabat) above which the core cools through thermal convection. The solid red line in panel $(\mathbf{d})$ is the thickness of the upper thermal boundary layer including the stagnant lid and the dashed red line is the stagnant lid thickness

\section{Specific Evolution Models}

\subsection{Earth}

The Earth is obviously the most studied of the terrestrial planets and serves as a test case for theories. Yet, many parts are still not understood and there is room for improvements. Many constraints are available for the Earth, concerning the structure, the present dynamics and the long term evolution of the core.

In terms of structure, the most important aspect is the existence of a solid inner core and a liquid outer core with the size of the inner core being very well known (Sect. 2). Moreover, the average stratification of seismic velocities, density, gravity, isentropic bulk modulus in the core is rather well constrained from seismology which helps to fix most parameters in the average state. Recent advances on the structure of the base of the mantle, in particular, the discovery of a new post-perovskite mineralogical phase may explain the seismic discontinuities in the "D" layer and may allow an estimate of the heat flow out of the core (Hernlund et al. 2005; Lay et al. 2008).

In terms of present dynamics, the Earth magnetic field has been systematically measured for a few centuries and high precision global maps have been obtained using satellites since 1980. Moreover, the determination of the time derivative of the magnetic field, the so-called secular variation, allows computing maps of the flow velocity at the top of the core (e.g., Hulot et al. 2002). This constrains the order of magnitude of fluctuations around the long 
term evolution of the averaged state and justifies the use of a separation of time scales as discussed in Sect. 3 above. Moreover, the time dependent magnetic field can be used to constrain dynamo models and to help derive scaling laws (Christensen and Tilgner 2004; Christensen and Aubert 2006; Aubert et al. 2009).

Finally, the long term evolution is constrained by paleomagnetic data that provide some insight into the characteristics of the main magnetic field in the past. The first important result from these studies is a proof of the existence of an early magnetic field. Tarduno et al. (2007) showed convincingly the existence of a magnetic field $3.2 \mathrm{Ga}$ ago, which to date is the oldest well-documented record of the Earth's magnetic field. Paleomagnetic data are difficult to come by; first because the number of available samples decreases with increasing age and second because old samples have often been magnetically reworked. Nevertheless, the available data show that the magnetic field has been in existence without significant (more than $100 \mathrm{Myr}$ ) interruption since that time. This observation provides a strong constraint on scenarios for Earth core evolution. For earlier times, beyond 3.2 Ga. b.p., no records are available which does not prove the absence of a magnetic field, of course. A proof of absence would be difficult to come by. Constraints could actually come from the chemistry of the lunar soil: Ozima et al. (2005) found that the amount of nitrogen in the lunar soil can best be explained by the implantation of gases from the Earth atmosphere. This would be easiest if it occurred before the geomagnetic field was strong enough to protect the atmosphere from erosion by interaction with the solar wind. They suggest that before $3.9 \mathrm{Gyr}$ ago, the geomagnetic field was very weak or even nonexistent.

Many models for the evolution of the Earth's core and the geodynamo have been proposed over the years. Two approaches are possible, both with pros and cons. The heat flow at the CMB is controlled by mantle convection and one would ideally develop a coupled self-consistent model to compute the evolution of both the core and the mantle. In addition to the obvious advantage of self-consistency, such a model would allow to use constraints other than those directly related to the core, such as the heat flow at the Earth's surface. A problem with this approach is that Earth's mantle convection by itself is not fully understood and the thermal evolution of the Earth is a subject of debate (e.g., Korenaga 2006; Jaupart et al. 2007; Labrosse and Jaupart 2007, for recent discussions). The heat flow at the CMB is highly uncertain (Lay et al. 2008) and the physics that controls it is controversially discussed (e.g., Labrosse 2002; Moore 2008). Therefore, these models may combine two ill-understood issues and discrepancies between model results and observations could result from both individually and from their interactions. The other option is to impose an evolution of the heat flow at the CMB and study the resulting evolution of the core.

The most comprehensive early study of the coupled mantle-core evolution is undoubtedly that by Stevenson et al. (1983) who calculated thermal evolution models for the mantles and cores of the terrestrial planets. They found that a thermal dynamo most likely was active in the early evolution of the Earth and that the inner core should have started to crystallize some 1.9-2.1 Gyr ago in order to reach the proper size at the present time. The onset of the inner core crystallization is accompanied by the start of compositional convection which leads to a higher ohmic dissipation (Sect. 3.2). Assuming that the large scale magnetic field scales as a square root of the ohmic dissipation, they cautiously speculate about a sharp increase of the core magnetic field upon inner core nucleation. Stevenson et al. caution that the change in the core field-if it occurred-would not necessarily be observable at the surface because of the unknown distribution of magnetic energy between the torroidal and poloidal components of the field. Mollett (1984) used a similar approach and included the effect of radioactivity in the core. The model approach has recently been revisited (Nimmo et al. 2004; Buffett 2002) in view of the progress in dynamo theory that has since been achieved. 
As has already been discussed in the preceding section, these models use a constant viscosity parameterization of mantle convection. The increase of computing power allowed the development of fully self-consistent calculations in which a cooling core model is coupled to a dynamical mantle convection model (Nakagawa and Tackley 2004, 2005). These authors find that heat sources in the core equivalent to $100 \mathrm{ppm}$ potassium would be required to sustain a dynamo for $3.5 \mathrm{Ga}$ and to arrive at the presently observed inner core radius. However, their model still has periods where the dynamo is inactive because of fluctuations of the CMB heat flow that occasionally drops below the adiabatic heat flow value. Including too much radiogenic heating would prevent growth of the inner core, or even make it remelt thereby frustrating compositional convection. Butler et al. (2005) and Costin and Butler (2006) also studied a coupled model with a full dynamical mantle and specifically included the effect of a stable layer at the base of the mantle enriched in radioactive elements as well as the effect of potassium in the core. They had the same difficulties in maintaining a dynamo throughout Earth's history while getting the inner core to reach the proper size at the present time. These authors also argue for some potassium in the core to help to solve the issue.

The difficulties faced by self-consistent approaches using either parameterized or full dynamical mantle convection models come mostly from the complexity of mantle convection. Therefore, the other approach of concentrating on the core is still valuable to test simple ideas on its evolution. In this approach, the heat flow at the CMB is imposed a priori, either constant or varying with time, and the evolution of the core and the dynamo is computed. Buffett et al. (1992) showed how to compute the growth of the inner core for a given heat flow at the CMB and found a rapid growth rate. Labrosse et al. (2001) further showed that the onset time for inner core crystallization is the solution of an equation that can be solved for any given heat flow history and concentration of radioactive elements. Because the inner core is a small fraction of the core, about $6 \%$ of its mass, the energy needed to be extracted from the core to grow to its present size is small and the required growth can be accomplished in less than $2 \mathrm{Ga}$. This time can, in principle, be lengthened if radiogenic heating is allowed in the core (e.g., Labrosse et al. 2001; Gubbins et al. 2004). However, a problem arises when the Ohmic dissipation rate is to be maintained. Since radiogenic heat is less efficiently converted into Ohmic dissipation (e.g. Roberts et al. 2003; Lister and Buffett 1995; Gubbins et al. 2003) than gravitational energy released upon inner core growth, the gain in Ohmic dissipation associated with the inclusion of radiogenic heat cannot compensate the loss in Ohmic dissipation associated with a smaller inner core growth rate. Thus, a certain level of Ohmic dissipation would require a certain growth rate, and thus determine the age of the inner core (Labrosse 2003; Nimmo et al. 2004). However, one should note that the rate of Ohmic dissipation required to explain the Earth's magnetic field is still uncertain.

Figure 4 shows the evolution of the radius of the inner core as function of time for an imposed constant CMB heat flow of 9 TW and without radiogenic heating in the core. The energy equation (8), in which all terms can be written as a function of the radius of the inner core $r_{\text {ic }}$ multiplied by its growth rate (provided the heat flow at the ICB is included in the secular cooling of the outer core, a very good approximation for a small inner core like that of the Earth, Labrosse et al. 2001), is used to compute the evolution of $r_{\text {ic }}$ and of each term in the equation, starting from the present situation backward in time. Then the efficiency equation (18) is used to obtain the evolution of the Ohmic dissipation and each of its contributions. The time evolution of the energy and entropy balances for the same calculation are shown in Fig. 5. All the physical parameters and the details of the calculation can be obtained from Labrosse (2003). 
Fig. 4 Radius of the inner core as function of time for a constant CMB heat flow of $9 \mathrm{TW}$

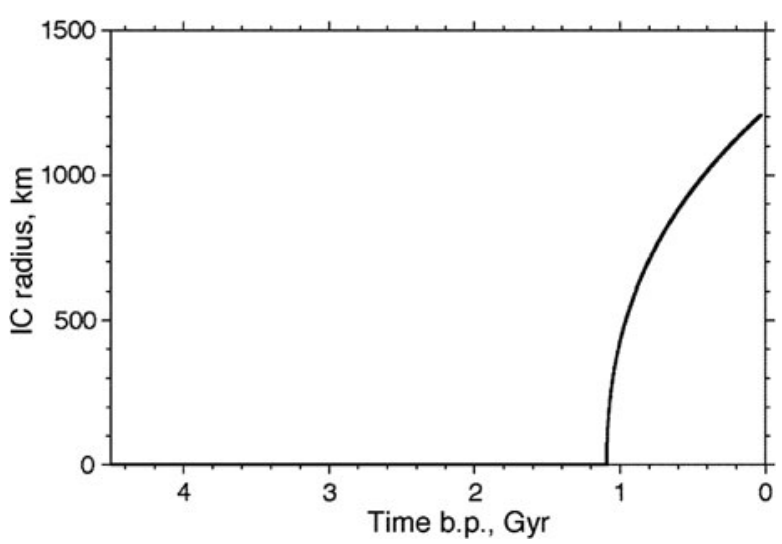

Fig. 5 Evolution of the power (upper panel) and entropy (lower panel) balances for a constant heat flow at the CMB of $9 \mathrm{TW}$.

Based on the model of Labrosse (2003)
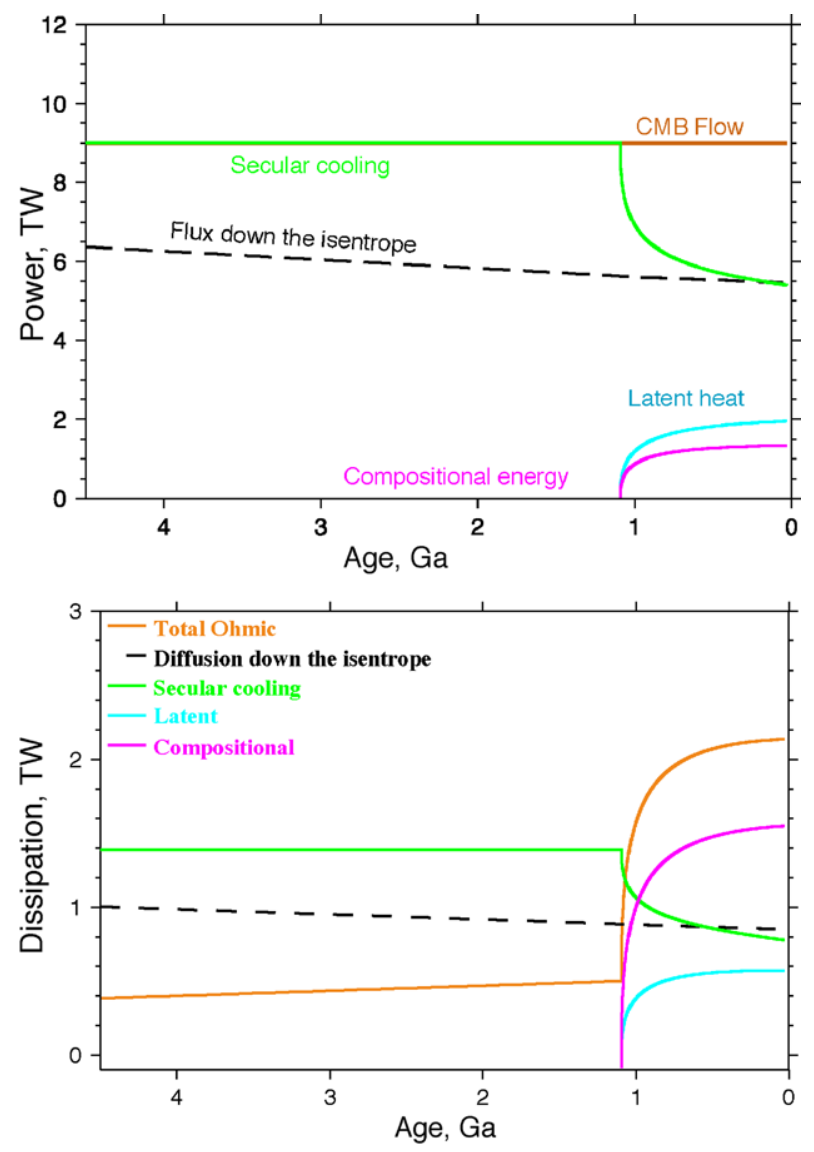

The growth of the inner core radius is found to follow a power law, $c(t)=c_{0}\left(t / t_{0}\right)^{\beta}$, with $1 / 3<\beta<1 / 2$ in this model and the age of the inner core is found to be about $1 \mathrm{Ga}$. The energy balance shows that when the inner core starts to crystallize, the secular cooling rate decreases as a consequence of the release of gravitational energy and latent heat that are 


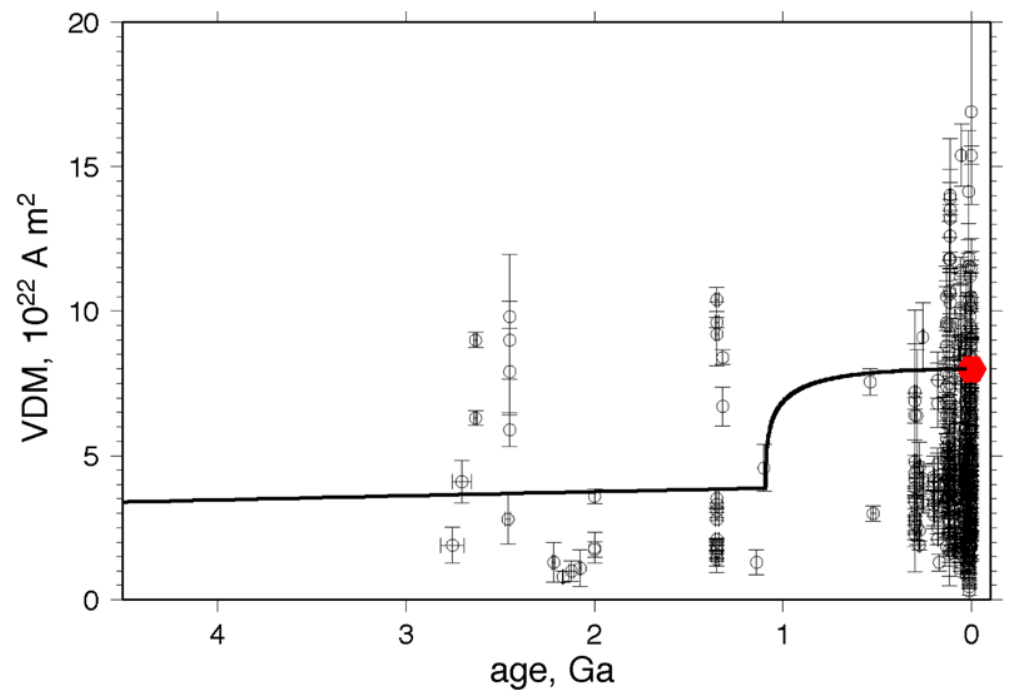

Fig. 6 Dipole moment of the Earth's magnetic field calculated for the models of Figs. 4 and 5 and compared to paleomagnetic data. The red hexagon is the present value and the dipole is assumed to scale as the square root of the ohmic dissipation

each presently of order $1 \mathrm{TW}$. Although gravitational energy contributes the smallest part of the power budget, it provides the largest contribution to the Ohmic dissipation as has been discussed in Sect. 3.2.

The onset of inner core growth results in a sharp increase in Ohmic dissipation, by a factor 4. It is then tempting to speculate on a sharp increase of the magnetic field that would allow the detection of the onset of inner core growth in the paleomagnetic record. However, the link between the Ohmic dissipation rate and the strength of the dipole, which dominates the paleomagnetic signal, is not obvious. The Ohmic dissipation rate is $\phi=\propto J^{2} \propto(\nabla \times \mathbf{B})^{2}$, which means that for a magnetic field spectrum $B(l)$ - with length scale $l-\phi(l) \sim B^{2}(l) / l^{2}$. If the increase of Ohmic dissipation occurred without affecting the shape of the spectrum, the total dissipation rate would be proportional to the square of the dipole field. On the other hand, dissipation usually occurs at small length-scales and the total dissipation rate could occur by increasing the energy at the smallest scales without changing the large scale dipole field. Stevenson et al. (1983) discussed the former option whereas Stevenson (1983) argued in favor of a large scale magnetic field that was independent of the Ohmic dissipation rate with an Elsasser number close to 1. More recently, the systematic investigation of dynamo models has allowed to develop scaling relationships in a rather wide parameter space (Christensen and Tilgner 2004; Christensen 2006; Christensen et al. 2009; Aubert et al. 2009) suggesting a RMS core magnetic field that scales with the power of $1 / 3$ of the Ohmic dissipation. This is a slightly smaller power than the scaling exponent of $1 / 2$ previously used. Figure 6 shows the evolution of the dipole field of Figs. 4 and 5 assuming that it scales as a square root of the dissipation rate and choosing the parameters such that the present dipole moment is obtained. The assumed power law thus maximizes the amplitude of the variations that are still smaller than the short term fluctuations found in the paleomagnetic record. This indicates that a detection of the onset of inner core growth is unlikely to result from studies of paleomagnetic intensities. 
Aubert et al. (2009) extended the study of the scaling of the average magnetic field to that of the dipole moment measured at the surface of the planet. They found that the onset of inner core crystallization, although making the Ohmic dissipation larger does not influence greatly the dipole moment because the magnetic field is generated deeper in the core for a compositional dynamo. The two effects balance out to make the dipole almost constant with time. This finding further complicates attempts to interpret Earth's magnetic record in terms of its core dynamics.

\subsection{Venus}

Since the first passage of Mariner 2 by Venus in 1962 at a distance of 6.6 planetary radii, it was clear that Venus does not have an Earth-like magnetosphere. The upper limit to the dipole moment obtained from the Pioneer Venus Orbiter placed the Venus intrinsic magnetic field at less than about $10^{-5}$ times that of Earth (Russell et al. 1979a, 1979b).

The lack of a present dynamo does not imply that Venus never had an intrinsic magnetic field although we have no information that relates directly to the past history of the field. The surface temperature of about $740 \mathrm{~K}$ is close to the Curie temperature of the main magnetic carrier minerals (magnetite, hematite, and pyrrhotite) of 850, 940, and $600 \mathrm{~K}$, respectively (e.g., Dunlop and Ozdemir 1997). Thus, temperature in the crust-possibly except for a thin near surface layer-is expected to be above the Curie point and any remanent crustal magnetic field remaining from an early period of dynamo activity should be weak if existent at all. It is important to note that the slow rotation of Venus (a Venus day of 243 Earth days is almost equal to the length of its year of $\sim 224$ Earth days and its sense of rotation is retrograde) does not exclude dynamo action. This appears to have been first noted by Hide (1956) and was recently reconfirmed by Christensen and Aubert (2006).

Thermal evolution models predict that there was an early magnetic field for Venus with a dipole moment of the same order as Earth's early field for about the first three billion years of Venus' history (Stevenson et al. 1983). During that time, thermal convection had driven the dynamo as in the other terrestrial planets. After the CMB heat flow had fallen below the adiabatic heat flow, the magnetic field ceased and did not rejuvenate; the core was too hot and the pressure was too low for inner core growth. The evolution models of Stevenson et al. (1983) were calculated using the constant viscosity parameterization and by simulating the stagnant lid through an increased, constant surface temperature of $1075 \mathrm{~K}$. This is the temperature at which silicate rock may be sufficiently weak to flow. The models cool more efficiently than models based on the scaling laws for stagnant lid convection. Assuming that Venus was in a stagnant lid regime throughout the entire evolution, the phase of early dynamo action would have most likely been shorter than predicted by Stevenson et al. because they should have overestimated core cooling.

One alternative scenario for the present lack of a Venus magnetic field is connected to the global crustal resurfacing event about 300-800 Ma ago (Schaber et al. 1992; McKinnon et al. 1997). Stevenson (2002) suggested that Venus's field ceased at that time due to a transition from a plate tectonics to a stagnant lid regime. The transition would have been followed by a period of warming of the mantle and the core and thermal convection and dynamo action in the core would have stopped. Even if Venus had an inner core at the time, inner core growth would have ceased with a warming mantle. The inner core may have started even to decrease in radius. The dynamo cannot be driven by chemical buoyancy in these circumstances.

If, on the other hand, the resurfacing was the consequence of a catastrophic overturn of the mantle or of lithosphere delamination (e.g., Turcotte 1993), Venus may have had a short-lived magnetic field during these times. During the catastrophic overturn, the interior 
Fig. 7 Four possible scenarios for the timing of internal magnetic field generation in Venus. The bluish regions indicate the time of dynamo generation. For further explanations, see the text

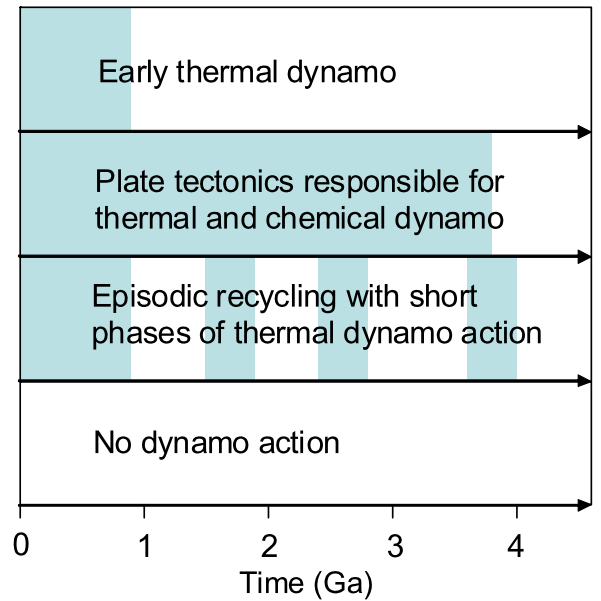

may have been cooled strongly and it is possible that the heat flow out of the core exceeded the critical isentropic core heat flow. This could have resulted in a short period of thermal convection and dynamo action in the core. Figure 7 summarizes the various scenarios of magnetic evolution postulated for Venus.

\subsection{Mars}

Mars today has no internally generated magnetic field, but the presence of a strong magnetization of the oldest parts of the crust (Acuña et al. 1998, 1999, 2001; Connerney et al. 1999) suggests that the planet generated a magnetic field early in its history. The inferred crustal magnetization of up to $\sim 10^{-30} \mathrm{~A} \mathrm{~m}^{-1}$, is an order of magnitude stronger than that of continental rock on Earth (Toft and Arkani-Hamed 1992; Arkani-Hamed and Dyment 1996). The magnetization is comparable in magnitude to the remanent magnetization of fresh extrusive basalt at oceanic ridges (Bleil and Petersen 1983).

One of the most effective ways to magnetize crustal rock is by thermo-remanent magnetization (TRM, Langlais et al. 2009, this issue). With TRM, the magnetization is produced when rock cools below the Curie temperature in the presence of a magnetizing field. There is a trade-off between the concentration of magnetic carriers and the strength of the magnetic field: The lower the magnetic field the more magnetic carriers are required to explain an observed magnetization. Assuming an early Martian magnetic field similar in strength to the present day Earth field, the required concentration of magnetic carriers in the Martian crust would be comparable to that in extrusive basalt. However, there is ample evidence that the $\mathrm{FeO}$ content of the Martian mantle is about twice that of the Earth's mantle (Sohl and Spohn 1997; Sanloup et al. 1999). Whether this high concentration of FeO translates to a high concentration of magnetic minerals depends on the oxidation state of the Martian mantle and lower parts of the crust. For a high concentration of magnetic minerals, the early magnetic field could have been smaller than that of the present Earth. In any case, the strength of the Martian magnetic anomalies suggests the existence of an intrinsic Martian magnetic field 0.1 to 10 times as strong as that of the present Earth (Ness et al. 1999, Mitchell et al. 1999, 2001).

The timing of the dynamo places constraints on the thermal evolution of the planet. The surface distribution of the magnetic anomalies suggests that the magnetization event predated the formation of the Hellas and Argyre basins (Connerney et al. 1999, 2004), roughly 


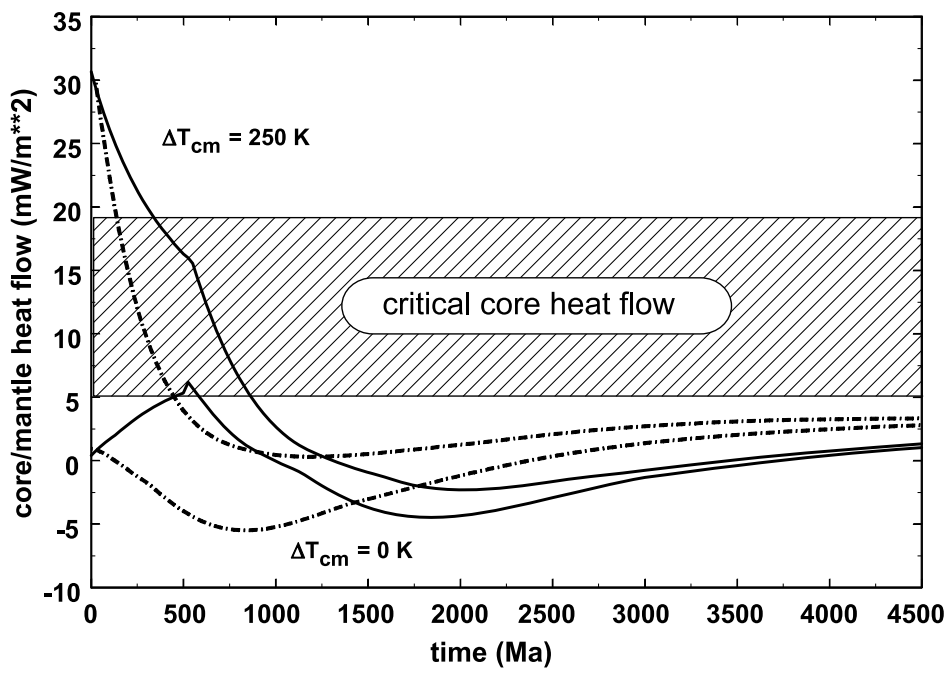

Fig. 8 Core-mantle heat flow as a function of time for models of Mars with early plate tectonics (solid line) and with stagnant lid convection throughout the entire evolution (dash-dotted line). The initial temperature differences assumed across the core-mantle boundary are $\Delta T_{\mathrm{cm}}=0$ and $\Delta T_{\mathrm{cm}}=250 \mathrm{~K}$ (from Breuer and Spohn 2003). An early magnetic field can be generated if the heat flow out of the core is larger than the critical core heat flow. Estimates of the latter range between 5 and $19 \mathrm{~mW} \mathrm{~m}^{-2}$

at the end of the Early Noachian (Head et al. 2001). It has been alternatively suggested that the dynamo turned on after the giant impact craters formed implying that the remanent magnetization of the crust was acquired later in Martian history (Schubert et al. 2000). This model, however, lacks an explanation for the strong magnetization of parts of the oldest crust.

An early dynamo is consistent with the results of most thermal evolution models that consider the magnetic field history (e.g., Schubert and Spohn 1990; Spohn et al. 1998, 2001b; Hauck and Phillips 2002; Breuer and Spohn 2003, 2006; Williams and Nimmo 2004). The models suggest an early thermal dynamo driven by rapid cooling of a core initially superheated with respect to the mantle (Fig. 8). In the subsequent evolution, the core remains liquid with a heat flow below the critical adiabatic value, and thus no dynamo action is observed. The core may remain completely molten if it contains at least $\sim 5 \mathrm{wt} .-\%$ sulfur and assuming a dry mantle rheology (Williams and Nimmo 2004). A core sulfur concentration of $14.2 \mathrm{wt} .-\%$ has been suggested based on chemical analyses of the SNC meteorites (Dreibus and Wänke 1985; McSween 1985).

It has been noted by Nimmo and Stevenson (2000) that a superheated core may not necessarily result from early differentiation and core formation. An alternative scenario thus assumes early rapid cooling of the core by plate tectonics and a transition to single-plate tectonics after about $500 \mathrm{Ma}$ (Nimmo and Stevenson 2000; Stevenson 2001). Connerney et al. (2005) have also argued for early plate tectonics. They noted two parallel great faults in the Terra Meridiani region with an offset of magnetic field contours similar to transform faults that occur in oceanic crust on Earth (Connerney et al. 2005). During the phase of plate tectonics, the interior cools efficiently and a thermal dynamo possibly followed by a chemical dynamo is conceivable. Soon after the transition to stagnant lid convection, however, the mantle warms and dynamo action stops (Fig. 8). Another model links the cessation of the Martian dynamo to the large impacts that formed the Hellas and Argyre basins. Roberts 
et al. (2009) suggested that impact heating associated with the largest basins (diameters $>2500 \mathrm{~km}$ ) could have caused the heat flow at the core-mantle boundary to decrease significantly by $10-40 \%$ which would have resulted in the cessation of the Martian dynamo.

One of the main characteristics of the Martian surface is its crustal dichotomy with the old heavily cratered crust in the southern hemisphere and a superficially younger crust in the northern hemisphere. This crustal dichotomy is accompanied by a difference in the magnetic field intensities in the two hemispheres: The northern hemisphere shows weak magnetic signals, whereas the southern hemisphere contains both strong and weak fields (Langlais et al. 2004). Explaining this difference by suggesting that the surface of the northern hemisphere is younger than the southern hemisphere and that it formed after the dynamo turned off is not entirely satisfying. The crust underneath the surface of the northern hemisphere is most likely as old as the crust of the southern hemisphere (Frey 2006). Thus, assuming a typical axial-dipole-dominated magnetic field and the northern and southern crust of similar composition and age, one would expect crustal fields of similar strength in both hemispheres. The magnetic dichotomy, therefore, requires post-dynamo mechanisms in the northern hemisphere that would have allowed removing its early remanent magnetization. Possible explanations are hydrothermal alteration (Solomon et al. 2005) or demagnetization resulting from early large impacts (e.g., Rochette et al. 2003; Mohit and Arkani-Hamed 2004). The strong correlation between the crustal and magnetic dichotomy also in their time of formation (both are believed to have been formed during the first $700 \mathrm{Ma}$ ) suggests a connection between the two processes. The crustal dichotomy if formed by internal dynamic processes requires a hemispheric-scale pattern of mantle flow. Several models exist to explain a degree-1 pattern that would also allow sufficiently vigorous core convection to sustain a short-lived dynamo. These include mantle convection in the presence of a deep perovskite phase transition layer close to the core-mantle boundary (Weinstein 1995; Harder and Christensen 1996; Buske 2006), mantle convection with strong radial viscosity variations (Zhong and Zuber 2001; Roberts and Zhong 2006), early magma ocean crystallization resulting in mantle overturn (Elkins-Tanton et al. 2003, 2005), or superplumes resulting from destabilization of the mantle lower thermal boundary layer (Ke and Solomatov 2006).

Degree-1 convection in the early evolution may even shape the magnetic field morphology at the surface. Stanley et al. (2008) show that variations of the core-mantle heat flow as a consequence of a low degree convection pattern could result in a single-hemisphere dynamo. This dynamo would produce strong magnetic fields in the southern hemisphere in case the heat flow out of the core below the southern hemisphere were stronger than that below the northern hemisphere. Such a dynamo would not need any post-dynamo processes to explain the observed magnetic field dichotomy and could also have had its impact on early atmospheric loss processes (Kulikov et al. 2007).

Stewart et al. (2007) have recently proposed that Mars - upon further cooling - may enter the snowing core regime described in more detail in Sect. 3.3. Whether or not convection driven by the falling iron crystals can drive a dynamo is not settled at this point in time, however.

\subsection{Mercury}

Mercury is exceptional among the terrestrial planets due to its large iron core that comprises about $80 \%$ of the planet's radius (e.g., Spohn et al. 2001a) and the presence of a weak magnetic field (Ness et al. 1975). The finding of a weak dynamo field by Mariner 10 has now been confirmed by MESSENGER (Anderson et al. 2008)

It is generally agreed that the Hermean magnetic field is likely generated in the core (Schubert et al. 1988), although a crustal source of the field can presently not be excluded 
(Aharonson et al. 2004). Thus, Mercury's magnetic field suggests that the planet's core should at least contain a fluid outer core layer. This conjecture has recently been supported by measurements of variations in Mercury's spin axis (Margot et al. 2007). A totally fluid core-without an inner core-is difficult to reconcile with the interior structure, thermal history models, and the magnetic field. The interior structure models suggest the mantle to be comparatively thin, about $600 \mathrm{~km}$, and thus heat may escape comparatively rapidly from the core. The heat flow from the core that is obtained from thermal history models (e.g., Stevenson et al. 1983; Schubert et al. 1988; Hauck et al. 2004; Buske 2006) is below the critical isentropic heat flow of about $11 \mathrm{~mW} \mathrm{~m}^{-1} \mathrm{~K}^{-1}$ (Schubert et al. 1988) and although the field is rather weak thermal convection in the core seems to be insufficient to drive the dynamo. It is even possible that the present mantle is not convecting and heat is only transported by conduction (Hauck et al. 2004). Thus, compositional buoyancy generated during inner core growth is likely necessary to produce the present day field.

As with the other terrestrial planets, the most likely candidate for the light alloying element in the core is sulfur but its concentration is basically unknown. Early planet formation models concluded that the Hermean core should contain none or only a small amount (a few percent) of sulfur because light elements would not condense at the orbital distance of Mercury close to the sun (e.g., Lewis 1972). Other formation models suggest efficient radial mixing of the accreting material (e.g., Wetherill 1988). Volatiles could thus have been transported from outer regions of the nebula toward the sun with the consequence of a possibly stronger concentration of sulfur in the Hermean core. Thermal evolution models together with the observed lobate scarps that suggest a small planetary contraction of about $2 \mathrm{~km}$ since the heavy bombardment of the planet (e.g., Strom et al. 1975) place some additional constraints on the concentration of sulfur in the core. Parameterized convection models by Hauck et al. (2004) assuming a non-Newtonian mantle rheology suggest a value between 5 and 8 wt.- $\%$ sulfur whereas the 2D and 3D thermal convection models with Newtonian rheology by Conzelmann (1999) and Buske (2006) can explain the observations with only 2 wt.- $\%$ of sulfur. Because of uncertainties in the radiogenic heat source concentration, the mantle rheology and the liquidus, it is difficult to derive firm bounds on the sulfur concentration from these models, however (Breuer et al. 2007).

Thermal evolution models (Stevenson et al. 1983; Schubert et al. 1988; Conzelmann 1999; Hauck et al. 2004; Buske 2006) suggest the following possible scenario for a dynamogenerated magnetic field. In the early evolution-possibly until 1-2 Ga b.p.- the dynamo would have been driven by thermal convection. The thermal dynamo would require the core to have been superheated with respect to the mantle (e.g., Breuer and Spohn 2003). It is possible that the dynamo ceased because the heat flow from the core became subadiabatic before an inner core started to grow. The onset time of inner core growth depends on various mantle convection parameters but increases with increasing sulfur content in the core (Hauck et al. 2004; Breuer et al. 2007). Since the time of the onset of inner core growth the Hermean dynamo should have been active until present if Mercury continued to cool steadily.

Early models of a hydromagnetic dynamo had problems, though, to explain the observed field strength which was found to be too weak in comparison with predictions (Stevenson et al. 1983; Schubert et al. 1988). Recent models have demonstrated that dynamos both in thin outer core shells (relative inner core size larger than about 0.8, Stanley et al. 2005) or in thick core shells (Heimpel et al. 2005) can produce weak magnetic fields, possibly consistent with Mercury's field. An alternative model of a thick shell dynamo has recently been suggested by Christensen (2006). In this model, the dynamo operates deep within the shell and is overlain by a stagnant fluid layer. A nonconvecting outer core layer may arise as a consequence of a subadiabatic CMB heat flow as has been obtained in the thermal 
evolution models of Hauck et al. (2004) and Buske (2006). The time-dependent magnetic field produced by the dynamo - as was shown by Christensen (2006) would then be strongly attenuated by the skin effect in the nonconvecting upper layer of the outer core.

Other alternative proposals for a weak magnetic field in Mercury include the thermoelectric dynamo (Stevenson 1987; Giampieri and Balogh 2002), the feedback dynamo (Glassmeier et al. 2007), and the snowing core dynamo (Chen et al. 2008). The thermoelectric dynamo makes use of a thermally-derived electromotive force set up at a distorted core-mantle boundary. Such a dynamo requires topography variations of the core-mantle boundary of the order of one kilometer due to mantle convection. The feedback dynamo model considers the feedback between magnetopause currents and a core dynamo. As Glassmeier et al. (2007) have shown for a simple alpha-omega dynamo model, the feedback between a magnetopause close enough to the planet (possibly closer than 2 planetary radii) and the dynamo may result in a weak external field. A variant of the snowing core dynamo model (see Sect. 3.3) has also been proposed for Mercury. Mercury is small enough such that its core is in the relevant pressure range (smaller than $40 \mathrm{GPa}$ ). As the inner core grows in a Fe-FeS core, sulfur becomes enriched in the outer core. The outer core composition may come close to the eutectic even if the overall sulfur content of the core is small (a few percent) and iron snow crystals may form near the CMB and sink toward the interior. Whether or not a dynamo could work driven by the falling iron snow is not well established as we have noted before, neither is it clear what the magnetic moment would be. Depending on details of the model, it is conceivable, however, that the power dissipated in the core under these circumstances will be relatively small.

The presently available magnetic field and other geophysical data do not allow a conclusion as to which dynamo may work in the planet. It is not even certain that an inner core exists although most workers in the field would consider that likely. New insights into the magnetic field evolution and the dynamo mechanism of Mercury are expected from future Mercury missions (e.g., Messenger and BepiColombo). In particular, the simultaneous measurements planned for the two spacecraft on the BepiColombo mission promise a better understanding of the sources of the Hermean magnetic field.

\subsection{Moon}

The Moon presently does not generate an internal magnetic field but paleomagnetic data, combined with radiometric ages of Apollo samples, suggests that a field of possibly $10^{4} \mathrm{nT}$ existed at 4.0 Ga decreasing to a few thousand nT at 3.2 Ga (Stephenson et al. 1975; Cisowski and Fuller 1986). Runcorn (1975) has argued that the lunar rocks were magnetized at the time of their formation by a field of internal origin. The easiest explanation for such a field is the operation of a dynamo in an iron-rich lunar core (e.g., Konrad and Spohn 1997; Spohn et al. 2001c; Stegman et al. 2003a, 2003b). The core of the Moon is, however, small (see Table 1 and the discussion in Sect. 2) which has been used as an argument against a dynamo (Runcorn 1975).

Some workers doubt that an internal dynamo is required to explain the magnetization of the Moon, and favor an alternative idea. The observed magnetic signature is suggested to be generated in association with large impacts during the early history (Hide 1972; Hood and Vickery 1984; Hood and Huang 1991; Hood et al. 2001). In this model, magnetization should be concentrated diametrically opposite to major impact basins in the presence of an ambient field. Satellite observations from the Lunar Prospector mission show concentrations of crustal magnetization at the antipodes of some but not all of the large impact structures (Purucker 2008). 
Assuming an internal origin of the lunar magnetic field, one must be aware that the paleomagnetic data show a gap of about $500 \mathrm{Ma}$ in the remanent magnetization of the lunar rock. There exist two alternative explanations for this gap: (1) the remanent magnetization of older basaltic rock was destroyed by impact gardening and/or the volcanic activity during the first $500 \mathrm{Ma}$ was very low or (2) the internal field started about $500 \mathrm{Ma}$ after core formation.

An early magnetic field has been suggested from thermal evolution models incorporating 2D and 3 D convection with strongly temperature dependent rheology (Konrad and Spohn 1997; Spohn et al. 2001c). These models show that a thermally driven dynamo might have been active from the beginning of core formation up to about $3 \mathrm{Ga}$ if the core was superheated by about $200 \mathrm{~K}$ with respect to the mantle. The models, however, neglect chemical stratification of the mantle due to the freezing of an early magma ocean.

A late onset of a dynamo may have been indirectly caused by the magma ocean (Alley and Parmentier 1998; Stegman et al. 2003a, 2003b). After crystallization of the magma ocean, a dense ilmenite and pyroxene cumulate located just underneath the KREEP layer may have sunk to the deep lunar interior and part of the KREEP layer may have been carried to depth together with the ilmenite layer. This mixed layer strongly enriched in radiogenic elements would have encircled the lunar core thermally insulating it from the rest of the mantle. Heat would have been trapped in the core and prevented the core from cooling and from powering a dynamo. The radiogenic heat sources would have heated the thermal blanket until it became thermally unstable and started to rise to the surface. With the removal of the thermal blanket, the core became able to convect vigorously and could have produced a short-lived magnetic field. An essential element of the model is the timing. The length of time that it takes the thermal blanket to heat up and rise back toward the surface is broadly consistent with two important events in early lunar history-the eruption of the mare basalts onto the lunar surface and the magnetization of lunar rocks. Even so, the model has some shortcomings. A Rayleigh-Taylor instability requires a substantial density difference between the ilmenite layer and the mantle for the ilmenite layer to sink rapidly enough into the deep interior (Parmentier et al. 2002). It is then difficult to heat this layer subsequently such that thermal buoyancy can overcome the compositional density difference barrier.

\subsection{Galilean Satellites and Titan}

One of the surprising discoveries of the Galileo mission was the detection of a dipolar, selfgenerated magnetic field at Ganymede (Kivelson et al. 1996), with an equatorial surface strength of about $720 \mathrm{nT}$ (Kivelson et al. 2002). No magnetic signals caused by permanent internal dipoles were found at the other major satellites of Jupiter, Io, Europa, and Callisto. Rather, Europa and Callisto have time varying fields that were interpreted as being caused by induction in subsurface oceans. An induced field component was also suggested for Ganymede since the dipolar field cannot fully explain the data. A small remaining component can either be explained as a quadrupole component of the self-generated magnetic field but best by an induced field (Kivelson et al. 2002). Cassini to date did not find evidence of magnetic fields-induced or self-sustained-for Titan (Backes et al. 2005) although it has been speculated to have an ocean (e.g., Hussmann et al. 2007, for a review).

The Galileo Ganymede magnetic field data were interpreted by Schubert et al. (1996) to conclude that the satellite should have a core and a dynamo. Other magnetic source processes, such as magneto-convection (e.g., Sarson et al. 1997), and remanent magnetism (Crary and Bagenal 1998) were ruled out mostly on the basis of the measured field strength that was too large to be explained by the alternatives. 


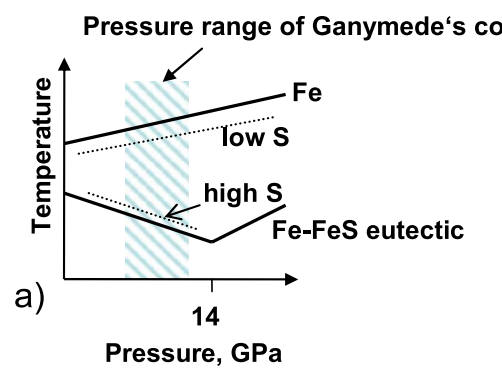

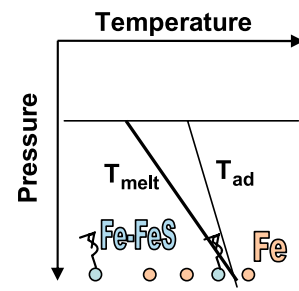

b)

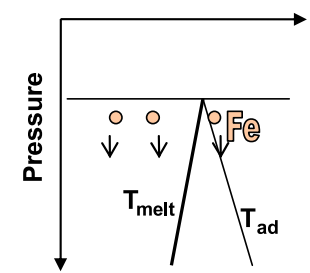

d)

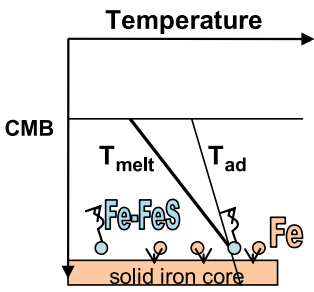

c)

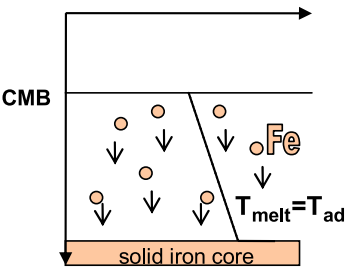

e)

Fig. 9 Schematic diagram showing the liquidus temperatures of $\mathrm{Fe}$ and eutectic $\mathrm{Fe}-\mathrm{FeS}$ with pressure (a) and sketches possible snowing core scenarios (b)-(e). In the pressure range of Ganymede's core, the eutectic temperature of $\mathrm{Fe}-\mathrm{FeS}$ decreases with pressure. As a consequence, $\mathrm{d} T_{\text {melt }} / \mathrm{d} P$ is positive for small sulfur concentrations and negative for sulfur concentrations close to the eutectic composition. Panels (b)-(e) show the isentrope $\left(T_{\text {ad }}\right)$ and the melting temperature ( $\left.T_{\text {melt }}\right)$ with pressure for two different cases: for small sulfur concentrations (b) and (c) and for close to eutectic concentrations (d) and (e). From (b) to (c) and (d) to (e), the core is cooling. For small sulfur concentrations $(\mathbf{b}),(\mathbf{c})$, precipitation starts in the center of the core and core convection is driven by the buoyancy of the light Fe-FeS liquid that forms close to the ICB (similar to the classical compositional driven convection in the Earth's core). For near eutectic sulfur concentrations (d), (e), precipitation of Fe starts close to CMB (d). Sinking iron snow may remelt and produce a gradient in the concentration of $\mathrm{Fe}$ ( $\mathrm{Fe}$ content increases with pressure) until the melting temperature becomes parallel to the isentropic temperature gradient-a solid inner iron core can then form (d). Precipitation of Fe at that stage may occur in the entire fluid core and may enforce compositional convection (adapted from Hauck et al. 2006)

A compositionally driven dynamo has been suggested for Ganymede by McKinnon (1996), Spohn and Breuer (1998), Hauck et al. (2006) and Bland et al. (2008), with the latter two discussing various forms of the snowing core model (compare Sect. 3.3). The snow model is particularly relevant for Ganymede because its core falls into the most relevant pressure range below $14 \mathrm{GPa}$. A summary of possible models and a schematic representation of the core melting diagram is given in Fig. 9. Depending on the sulfur content, the core composition may be on the Fe-rich side of the eutectic or on the FeS-rich side and fluid motion may be induced by either Fe-snow sinking from regions close to the core-mantle boundary or by FeS-snow floating upward from the deep core. The sulfur content of the core is basically unknown. Kuskov and Kronrod (2001) demonstrate that a eutectic composition of about 20 weight- $\%$ as well as a low sulfur core is consistent with the geophysical data. However, models of the Jovian nebula and the dominance of sulfur on the surface of Io argue against a sulfur poor composition (e.g., Schubert et al. 2004, for a review). The unknown oxidation state of the satellite interiors during core formation precludes an exact knowledge of the composition of the cores of the satellites (e.g., Scott et al. 2002), however, even if the bulk composition were known. A further uncertainty in the modeling comes from the unknown temperature and thermal history of the core and details of the core power balance. 
Both Hauck et al. (2006) and Bland et al. (2008) consider power balances for the dynamo similar to (8) above and assume that the core is initially molten. Both require the silicate mantle to cool the core sufficiently rapidly to arrive at a sufficiently large cooling power $Q_{\mathrm{c}}$. Sufficient cooling is possible if the rheology of the mantle is close to that of a wet olivine mantle. In addition, both require a freezing core with latent heat and gravitational energy contributing to the power balance. For iron-rich compositions, Hauck et al. (2006) allow a maximum of 13 weight- $\% \mathrm{~S}$ to arrive at a freezing core. Bland et al. (2008) argue that latent heat in the iron snowing core model would not be available to drive the dynamo since it would be released immediately below the CMB and should be effectively removed. Therefore, Bland et al. accept models only where the latent heat is released deep in the core. These authors conclude that this would require the core to have less than 3 weight- $\%$ sulfur. At least the latter bound on the sulfur content is difficult to reconcile with cosmochemical models.

A purely thermal dynamo has recently been suggested by Kimura et al. (2009). These authors assume an initially cold interior (initial core temperature close to the eutectic temperature of about $1250 \mathrm{~K}$ ) that would have heated with time as a consequence of radiogenic decay and only recently would have started to cool by thermal convection. The latter would drive the dynamo. The model would work best for a core with a composition close to the eutectic. In that case, the radius of the core would be maximized and the thickness of the mantle minimized, allowing most efficient cooling. One should note, however, that Kimura et al. (2009) assume a critical isentropic heat flow of only $1 \mathrm{~mW} \mathrm{~m}^{-2}$ which is even smaller than the minimum possible value considered by Hauck et al. (2006) and Bland et al. (2008).

The difficulty of generating a dynamo in Ganymede either through thermal or chemical convection led Stevenson (1996) and Showman et al. (1997) to suggest that a period of tidal heating in Ganymede's past may have blocked the cooling of the core for some time, and thus may have enabled the generation of the present-day field. The necessary tidal heating would have been the consequence of a resonance passage that resulted in an excitation of the satellite's eccentricity (Showman and Malhotra 1997). If sufficient tidal dissipation occurred in Ganymede's silicate mantle, an increasing temperature would have prevented the core from cooling until the resonance passage ended. After escape from the resonance, rapid mantle and core cooling would have triggered dynamo action via thermal and/or compositional convection. Bland et al. (2008), however, argue that tidal heating in Ganymede's mantle may have never sufficed to cause the thermal runaway necessary to prevent core cooling. Another variant of the delayed core cooling model is that of Spohn and Breuer (1998) who suggest that Ganymede may have accreted cold (with temperature decreasing with depth as in classical accretion scenarios) and differentiation and core formation may have been caused by warming of the interior over a few $\mathrm{Ga}$ as a consequence of radiogenic heating and heat trapping. The core would thus have formed late and core cooling and dynamo action may be a recent feature for this satellite.

The lack of a present internal dynamo in Io, Callisto, and perhaps Titan can be more easily understood. Callisto's moment-of-inertia factor suggests a largely undifferentiated (or partly differentiated) satellite-and there would be no iron-rich core in which a dynamo could be generated. A similar explanation may apply to Titan if it could be confirmed that it does not have a self-sustained magnetic field. Models of the early evolution and differentiation of the satellite (e.g., Kirk and Stevenson 1987; Lunine and Stevenson 1987; Tobie et al. 2005) suggest that it did not differentiate to form an iron-rich core.

The case for Europa is less clear, in particular when comparing Europa with Ganymede. It is widely believed that Europa has an interior structure similar to that of Ganymede with a core, a silicate mantle, and an ice layer (Sohl et al. 2002), although the absence of a 
self-sustained magnetic field makes the case for a core in Europa less compelling. As a consequence, one would expect a similar thermal and magnetic evolution for both satellites. Thermal evolution models suggest that it is even more likely for Europa to generate an internal field if the same set of parameter values for mantle rheology and radioactive heat source density is used as for Ganymede (Breuer et al. 2008). Accepting core convection in Ganymede, the question than poses itself of how to explain the absence of core convection in Europa. Three possible explanations are: (1) Europa has no iron core. This is (marginally) consistent with the gravity observation but leaves the question open why Ganymede should have fully differentiated while Europa did not. (2) A higher concentration of light elements in Europa's core. Taking sulfur as a point in case, Europa may have more sulfur (on the ironrich side of the eutectic), in which case more cooling would be required to freeze the core. (3) Tidal heating in the silicate mantle of Europa. This is certainly possible if the mantle is partially molten which is suggested at least for the early evolution in thermal evolution models (Breuer et al. 2008). In fact, only a few times the present-day radiogenic heating rate would be required to possibly frustrate dynamo action. This much tidal heat is consistent with the models of Hussman and Spohn (2004).

Finally, we note that the absence of a magnetic field of Io is best explained by tidal heating in the mantle blocking the heat flow from the core (Wienbruch and Spohn 1995; see also Schubert et al. 2004, for a discussion).

\section{Summary and Discussion}

A magnetic field of internal origin — as we have discussed in the present paper-is a distinguishing feature between terrestrial planets and satellites that may reveal properties of their deep interiors. Necessary conditions for the existence of these fields are electrically conducting regions in the interior with material flow, a sufficiently strong power source to drive a dynamo, and sufficiently strong rotation to promote helicity of the flow and large scale magnetic field generation (compare Busse and Simitev 2007; Christensen and Wicht 2007; Stanley and Glatzmeier 2009; Christensen 2009). The candidate regions in terrestrial planets and satellites are the iron-rich cores. Salty water oceans in icy satellites could in principle also serve as dynamo regions but at least for the icy satellites of Jupiter this possibility has been ruled out on the basis of considerations of the power necessary to drive a dynamo (e.g., Schubert et al. 1996).

The existence of cores in Earth, Mars, and Mercury are proven beyond any reasonable doubt. Although there is no conclusive evidence for an iron-rich core in Venus, it is reasonable to assume a core for that planet as well because of its similarity in size and mass to the Earth. For the satellites, the situation is not as clear cut. The available data suggest that the lunar core is small and it cannot be completely ruled out that there is no iron core at all. Strong cases can be made for cores in Io and Ganymede; but models without cores are possible for Europa, albeit not required. Callisto is likely to lack a core. The available evidence suggests that this satellite is incompletely differentiated. However, it must be kept in mind that the models of the interior structures of these satellites assume hydrostatic equilibrium, an assumption that may be problematic given their small sizes and masses. No conclusive evidence for or against cores in Titan, Triton, Pluto, and Vesta is available. There is hope that the Cassini mission will eventually provide data for Titan and the Dawn and New Horizons missions may do so for Vesta and Pluto, respectively.

Rotation is a necessary condition for dynamo action to promote helicity and large scale magnetic field. Balancing the Lorentz and Coriolis forces has resulted in a semi-empirical 
prediction of the dipole moments of the planets known as Busse's law (Busse 1976). It is also sometimes postulated that the lack of a magnetic field of Venus may be explained by its small rotation rate. However, Hide (1956) already noted that even the Coriolis force in Venus' core is strong enough to effect large scale flow. Stevenson et al. (1983) have used a power balance for the core to estimate the dipole moment. The recent work by Christensen and Aubert (2006) has confirmed the notion that the strengths of the planetary magnetic fields should be dependent on the power balance of the core and only in extreme cases on the planetary rotation rate.

The power balance of the cores is largely dependent on the heat transfer rate in the mantle. Regardless of whether the dynamo is driven by thermal or compositional buoyancy, the core must be cooling to induce convection. This requires the heat flow from the core to exceed the heat flow conducted along the core adiabat (or isentrope) for thermal convection. The critical heat flow depends as we have discussed on the temperature of the core, gravity, and the core thermodynamic and transport properties, in particular, the thermal expansion coefficient, the density, the specific heat, and the thermal conductivity. The adiabatic temperature gradient can also be cast in terms of the Grüneisen parameter and the bulk modulus. In any case, the threshold heat flow increases with planetary mass from a few $\mathrm{mW} \mathrm{m}^{-2}$ for Ganymede to reach a few tens of $\mathrm{mW} \mathrm{m}^{-2}$ for Earth and Venus. No threshold for the core-mantle heat flow is expected for compositional convection. The latent heat must be removed but it will simply reduce the rate of inner core growth. Inner core growth requires, however, that the temperature in the core falls below the liquidus which in turn depends on pressure and core composition. Both, the thermal and the compositionally driven dynamo will have to overcome Ohmic dissipation (e.g., Gubbins et al. 2003; Christensen and Tilgner 2004).

The rate of heat transfer in the mantle depends largely on mantle rheology, its physical, thermodynamic and transport properties, and its composition. We will not discuss conduction here since conduction is unlikely to provide sufficient cooling on planetary scales. Rather, we will restrict ourselves to discuss convection. Among the physical properties, the most important ones are the thickness of the mantle and gravity. While the thickness of the mantle cancels out in constant viscosity mantle convection heat transfers scaling laws, it is retained in variable viscosity scaling laws. Gravity immediately affects the vigor of mantle convection. More important than these parameters are the rheology parameters. Since the viscosity of rock is strongly temperature dependent, the viscosity can vary by many orders of magnitude and the heat transfer rate can vary by substantial factors. Moreover, the temperature dependence of viscosity provides for a thermostat effect and determines the style of mantle convection. In addition to the temperature dependence, the rheology is dependent on the chemistry of the planetary mantle, in particular on the concentration of volatiles. Although it is not well established why the Earth has plate tectonics and the other planets have not, it is widely held that at least part of the answer lies with the availability of water on the planet. In any case, numerical simulations of mantle convection have shown that plate tectonics can cool the deep interior of a planet much more effectively than stagnant lid convection. A simple reasoning shows why: For plate tectonics, the surface plates participate in the convection and the convective heat transfer cycle operates between the surface temperature and the core-mantle boundary temperature. In stagnant lid convection, the convective heat transfer cycle operates between the temperature below the lid (about $1100 \mathrm{~K}$ ) and the core-mantle boundary temperature.

Although there are a large number of uncertain parameter values and, therefore, a large number of possible models, a simple model can account for the differences in the magnetism of the terrestrial planets. The two planets, Earth and Mercury, that do have self-sustained magnetic fields at the present time have inner cores. This has been proven for the Earth and is 
likely for Mercury given its presumed refractory composition (e.g., Woods et al. 1981; Lewis 1988). Both planets have comparatively large heat transfer rates through their mantles; the Earth because of its plate tectonics and Mercury because of its thin mantle. In addition, the liquidus temperature in the Hermean core should be comparatively large, given its presumed refractory composition. The other two, Mars and Venus, may lack inner cores. Their core to mantle radii ratios are around 0.5 (as compared to 0.8 for Mercury) and both are likely to lack plate tectonics. In addition, Mars' core has a low liquidus temperature if it is indeed close to eutectic in composition. Both planets may have had thermally driven dynamos in their early evolutions but the heat flow from the mantle may have become subcritical during their later evolutions (e.g., Stevenson et al. 1983; Schubert and Spohn 1990; Breuer and Spohn 2003; Hauck et al. 2004). However, more exotic models are also possible (e.g., Stevenson 2001). For instance, it is possible that these planets do have inner cores that are not growing at the present time. Thermal history calculations show that the core of Mars may have been warming after cooling through an early phase of plate tectonics (Nimmo and Stevenson 2000; Breuer and Spohn 2003) and Venus may undergo some episodic form of mantle convection with surface foundering events throughout its history (e.g., Turcotte 1993).

The Moon may be explained in a simple scenario just like Mars and Venus. If it has a core, than the relative core radius at 0.2 is smaller than that of Mars and Venus and heat transfer through the mantle should be even smaller. Thus, the Moon may have had an early dynamo that died a few hundred million years to a billion years after formation (Konrad and Spohn 1997; Spohn et al. 2001c).

The interesting case is Ganymede. Although the multitude of parameters certainly allows the construction of models that predict a dynamo for the satellite, the task becomes more difficult if the model has to explain the lack of fields on Mars and Europa at the same time. If Ganymede is to work like Mars and Venus, then the heat transfer from the core is small. The ratio between the core radius and the radius of the silicate shell is about 0.5 just as for the two planets. In addition, Ganymede has a thick ice shell that would further insulate the deep interior. Ganymede's core is also likely to be sulfur rich, around $21 \mathrm{wt} .-\%$ if it is chondritic in composition. This will reduce its likelihood to freeze an inner core.

In Fig. 10, we graphically display the possible evolution of the simple model. The plot qualitatively displays the planets in a plane defined by the concentration of light elements in the core as an indicator of the time needed to cool to the liquidus temperature (the higher the concentration, the longer the time needed to cool to the liquidus) and the heat transfer rate through the mantle. Figure 10a shows the situation for the planets in the early solar system. Most of the cores are liquid and convecting but Mercury has begun freezing an inner core mostly because of its lack of light constituents. The planets have magnetic fields, thermally driven for Venus, Earth, and Mars, and compositionally driven for Mercury. Figure 10b portrays the present-day situation. Mars and Venus still have liquid cores but the thermally driven dynamo has died off. Earth is growing an inner core and has a compositionally driven dynamo. Mercury has almost frozen its core and the inner core growth rate has substantially decreased. Accordingly, consistent with Stanley et al. (2005) or Christensen (2006), its magnetic field has weakened. In Fig. 10c, some time into the future of the solar system, Mars and Venus have begun freezing inner cores. Both planets may thus have restarted their dynamos this time compositionally driven. Earth has frozen most of the core; the growth rate has decreased as has the strength of the dynamo. Mercury has frozen the core completely.

It is interesting to speculate on Earth-like Exoplanets. There is no simple way to predict whether or not such a planet will have a magnetic field. In view of its habitability a long lasting field would be advantageous to allow the evolution of life on the surface. The evidence from the solar system certainly suggests that plate tectonics will be helpful. The scientific 
Fig. 10 Simple model of the evolution of the magnetism of the terrestrial planets. Vertical dashed areas are regions where the dynamos are driven by compositional convection resulting from a growing inner core. In the hashed area, the models have large inner cores with small growth rates and weak magnetic fields. The red line marks the onset of inner core growth. The black line marks the transition to large inner cores.

The blue line indicates the cessation of the dynamo for a complete frozen core. Time increases from (a) to (c) where (a) indicates a time in the early evolution, (b) the present day, and (c) a time in the future. The positions of the planets are indicated with stars and depend on the assumed amount of light elements in the core and the efficiency of cooling. Note that the borders between regimes are, of course, schematic. For further explanation, see the text
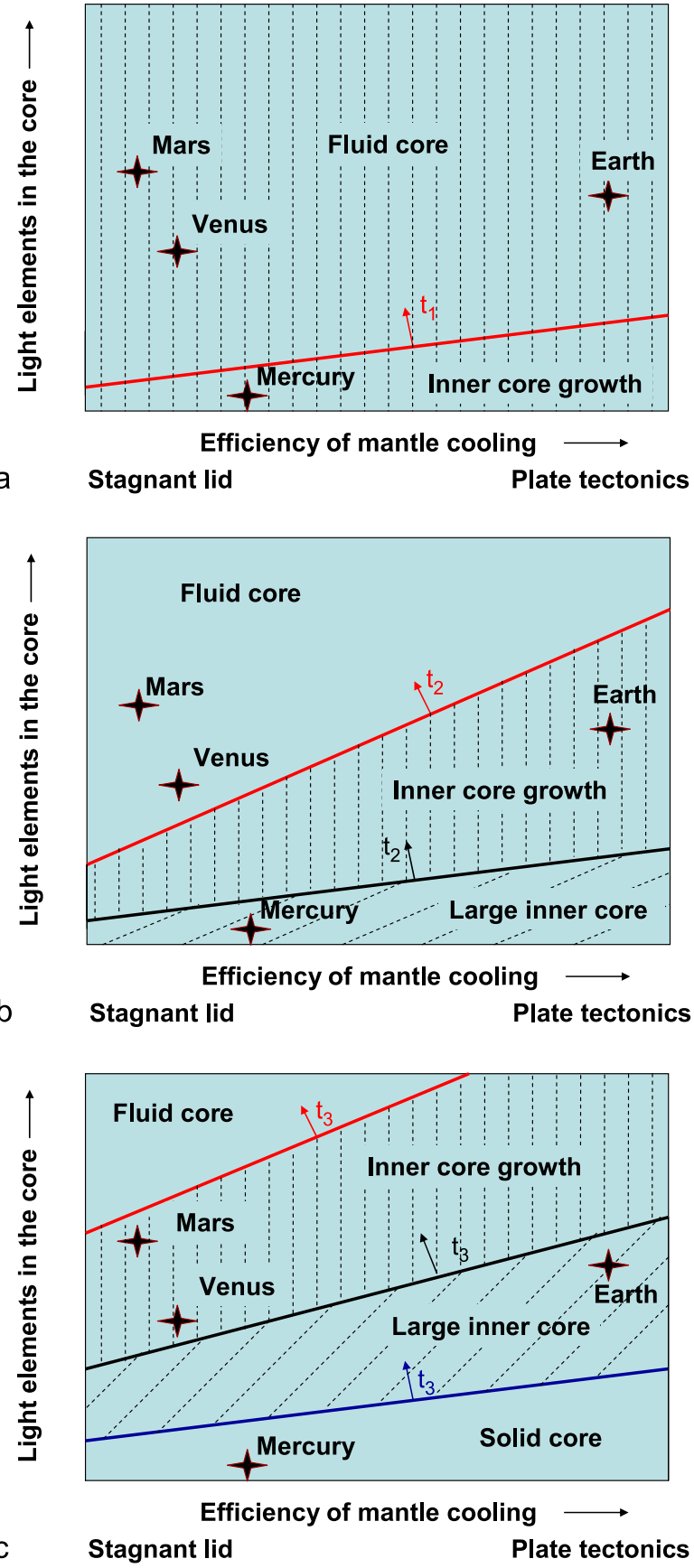

community presently debates whether or not super Earths would be expected to have plate tectonics or not. The debate has not settled with, e.g., O'Neill and Lenardic (2007) arguing against and Valencia et al. (2007) and Valencia and O'Connell (2009) arguing for plate tectonics. It is to be expected that the threshold for a thermally driven dynamo will increase 
with pressure because the adiabatic gradient is expected to increase but the variation of the melting point depression due to, e.g., sulfur beyond $25 \mathrm{GPa}$ is unknown.

A better understanding of the magnetism of the terrestrial planets and satellites certainly requires a better knowledge of material properties and their variations with pressure and temperature. This can be achieved - at least to some extent-by progress in laboratory techniques. However, even more important than that-it seems to us-would be in-situ data from planets and satellites. These include geodetic and geophysical data from hitherto unexplored bodies such as Vesta and Pluto but also and perhaps even more importantly conclusive data on the cores of the closer to home planets Mars, Venus, and Mercury; how large are their cores and their inner cores, if there are any. And, last but not least, a better exploration of the magnetic fields of Mars and Mercury.

\section{References}

M.H. Acuña, J.E.P. Connerney, N.F. Ness, R.P. Lin, D. Mitchell, C.W. Carlson, J. McFadden, K.A. Anderson, H. Rème, C. Mazelle, D. Vignes, P. Wasilewski, P. Cloutier, Magnetic field and plasma observations at Mars: Initial results of the Mars global surveyor mission. Science 279, 1676-1680 (1998)

M.H. Acuña, J.E.P. Connerney, N.F. Ness, R.P. Lin, D. Mitchell, C.W. Carlson, J. McFadden, K.A. Anderson, H. Rème, C. Mazelle, D. Vignes, P. Wasilewski, P. Cloutier, Global distribution of crustal magnetism discovered by the Mars global surveyor MAG/ER experiment. Science 284, 790-793 (1999)

M.H. Acuña, J.E.P. Connerney, P. Wasilewski, R.P. Lin, D. Mitchell, K.A. Anderson, C.W. Carlson, J. McFadden, H. Rème, C. Mazelle, D. Vignes, S.J. Bauer, P. Cloutier, N.F. Ness, Magnetic field of Mars: Summary of results from the aerobraking and mapping orbits. J. Geophys. Res. 106(E10), 23,40323,418 (2001)

O. Aharonson, M.T. Zuber, S.C. Solomon, Crustal remanence in an internally magnetized non-uniform shell: A possible source for Mercury's magnetic field? Earth Planet. Sci. Lett. 218, 261-268 (2004)

K.M. Alley, E.M. Parmentier, Numerical experiments on thermal convection in a chemically stratified viscous fluid heated from below: implications for a model of lunar evolution. Phys. Earth Planet. Int. 108, 15-32 (1998)

B.J. Anderson, M.H. Acuña, H. Korth, M.E. Purucker, C.L. Johnson, J.A. Slavin, S.C. Solomon, R.L. McNutt, The structure of Mercury's magnetic field from MESSENGER's first flyby. Science 321, 82-85 (2008). doi:10.1126/science.1159081

J.D. Anderson, E.L. Lau, W.L. Sjogren, G. Schubert, W.B. Moore, Gravitational constraints on the internal structure of Ganymede. Nature 384, 541-543 (1996)

J.D. Anderson, E.L. Lau, W.L. Sjogren, G. Schubert, W.B. Moore, Europa's differentiated internal structure: Inferences from two Galileo encounters. Science 276, 1236-123 (1998)

J.D. Anderson, R.A. Jacobson, T.P. McElrath et al., Shape, mean radius, gravity field and interior structure of Callisto. Icarus 153, 157-161 (2001a). doi:10.1006/icar.2001.6664

J.D. Anderson, R.A. Jacobson, E.L. Lau, W.B. Moore, G. Schubert, Io's gravity field and interior structure. J. Geophys. Res. 106(E12), 32,963-32,969 (2001b)

A. Anselmi, G. Scoon, BepiColombo, ESA's Mercury Cornerstone mission. Planet. Space Sci. 49, 1409-1420 (2001)

J. Arkani-Hamed, J. Dyment, Magnetic potential and magnetization contrasts of Earth's lithosphere. J. Geophys. Res. 101(B5), 11,401-11,426 (1996)

Y. Asahara, D.J. Frost, D.C. Rubie, Partitioning of FeO between magnesiowustite and liquid iron at high pressures and temperatures: Implications for the composition of the Earth's outer core. Earth Planet. Sci. Lett. 257, 435-449 (2007)

J. Aubert, S. Labrosse, C. Poitou, Modelling the paleo-evolution of the geodynamo. Geophys. J. Int. 179, 1414-1428 (2009). doi:10.1111/j.1365-246X.2009.04361.x

H. Backes, F.M. Neubauer, M.K. Dougherty, N. Achilleos, N. André, C.S. Arridge, C. Bertucci, G.H. Jones, K.K. Khurana, C.T. Russell, A. Wennmacher, Titan's magnetic field signature during the first Cassini encounter. Science 308, 992-995 (2005)

P.S. Balog, R.A. Secco, D.C. Rubie, D.J. Frost, Equation of state of liquid Fe-10 wt.\% S: Implications for the metallic cores of planetary bodies. J. Geophys. Res. 108(B2), 2124 (2003). doi:10.1029/2001JB001646

Basaltic Volcanism Study Project (BVSP), Basaltic Volcanism on the Terrestrial Planets (Pergamon, New York, 1981). 
D. Bercovici, Generation of plate tectonics from lithosphere-mantle flow and void-volatile self-lubrication. Earth Planet. Sci. Lett. 154, 139-151 (1998)

C.M. Bertka, Y. Fei, Mineralogy of the Martian interior up to core-mantle boundary pressures. J. Geophys. Res. 102, 5251-5264 (1997)

M.T. Bland, A.P. Showman, G. Tobie, The production of Ganymede's magnetic field. Icarus 198, 384-399 (2008)

U. Bleil, N. Petersen, Variations in magnetization intensity and low-temperature titanomagnetite oxidation of ocean floor basalts. Nature 301, 384-388 (1983)

J.R. Booker, K.C. Stengel, Further thoughts on convective heat transport in a variable viscosity fluid. J. Fluid Mech. 86, 289-291 (1978)

S.I. Braginsky, Mac-oscillations of the hidden ocean of the core. J. Geomag. Geoelectr. 45, 1517-1538 (1993)

S.I. Braginsky, Formation of the stratified ocean of the core. Earth Planet. Sci. Lett. 243, 650-656 (2006)

S.I. Braginsky, P.H. Roberts, Equations governing convection in Earth's core and the geodynamo. Geophys. Astrophys. Fluid Dyn. 79, 1-97 (1995)

D. Breuer, W.B. Moore, Dynamics and thermal history of the terrestrial planets, the Moon, and Io, in Planets and Moons, ed. by T. Spohn. Treatise on Geophysics, vol. 10 (Elsevier, Amsterdam, 2007), pp. 299-348

D. Breuer, T. Spohn, Early plate tectonics versus single-plate tectonics: Evidence from the magnetic field history and crust evolution. J. Geophys. Res.-Planets 108(E7), 5072 (2003). doi:10.1029/20002JE001999

D. Breuer, T. Spohn, Viscosity of the Martian mantle and its initial temperature: Constraints from crust formation history and the evolution of the magnetic field. Planet. Space Sci. 54, 153-169 (2006)

D. Breuer, S.A. Hauck, M. Buske, M. Pauer, T. Spohn, Interior evolution of Mercury. Space Sci. Rev. 132(24), 229-260 (2007). doi:10.1007/s11214-007-9228-9

D. Breuer, H. Hussmann, T. Spohn, The magnetic dichotomy of the Galilean satellites Europa and Ganymede, in Geophysical Research Abstracts, European Geosciences Union-General Assembly, Vienna (Austria), 2008-04-13-2008-04-18, abstract 07511, 2008

B.A. Buffett, Estimates of heat flow in the deep mantle based on the power requirements for the geodynamo. Geophys. Res. Lett. 29, 7 (2002)

B. Buffett, Core-mantle interactions, in Treatise on Geophysics, ed. by G. Schubert (Elsevier, Amsterdam, 2007), pp. 345-358

B.A. Buffett, H.E. Huppert, J.R. Lister, A.W. Woods, Analytical model for solidification of the Earth's core. Nature 356, 329-331 (1992)

B.A. Buffett, H.E. Huppert, J.R. Lister, A.W. Woods, On the thermal evolution of the Earth's core. J. Geophys. Res. 101, 7989-8006 (1996)

M. Buske, Dreidimensionale thermische Evolutionsmodelle für das Innere von Mars und Merkur, Doktorarbeit, IMPRS, Katlenburg-Lindau, 2006

F.H. Busse, Phys. Earth Planet. Inter. 12, 350-358 (1976)

F.H. Busse, R. Simitev, Planetary dynamos, in Planets and Moons, ed. by T. Spohn. Treatise on Geophysics, vol. 10 (Elsevier, Amsterdam, 2007), pp. 281-290

S. Butler, W. Peltier, S. Costin, Numerical models of the Earth's thermal history: Effects of inner-core solidification and core potassium. Phys. Earth Planet. Inter. 152, 22-42 (2005)

B. Chen, J. Li, S.A. Hauck, Non-ideal liquidus curve in the Fe-S system and Mercury's snowing core. Geophys. Res. Lett. 35 (2008). doi:10.1029/2008GL033311

U.R. Christensen, Thermal evolution models for the Earth. J. Geophys. Res. 90, 2995-3007 (1985)

U.R. Christensen, A deep dynamo generating Mercury's magnetic field. Nature 444, 1056-1058 (2006). doi: 10.1038/nature05342

U. Christensen, Dynamo scaling laws and applications to the planets. Space Sci. Rev. (2009). doi:10.1007/ s11214-009-9553-2, this issue

U.R. Christensen, J. Aubert, Geophys. J. Int. 166(1), 97-114 (2006)

U.R. Christensen, A. Tilgner, Power requirement of the geodynamo from ohmic losses in numerical and laboratory dynamos. Nature 429, 169-171 (2004)

U.R. Christensen, J. Wicht, Numerical dynamo simulations, in Core Dynamics, ed. by P. Olson. Treatise on Geophysics, vol. 8 (Elsevier, Amsterdam, 2007), pp. 245-282

U.R. Christensen, V. Holzwarth, A. Reiners, Energy flux determines magnetic field strength of planets and stars. Nature 457, 167-169 (2009)

L. Chudinovskikh, R. Boehler, Eutectic melting in the system Fe-S to $44 \mathrm{GPa}$. Earth Planet Sci. Lett. 257, 97-103 (2007). doi:10.1016/j.eps1.2007.02.024

S.M. Cisowski, M. Fuller, Lunar paleointensities via the IRMs normalization method and the early magnetic history of the Moon, in Origin of the Moon, ed. by W.K. Hartmann, R.J. Phillips, G.J. Taylor (Lunar and Planetary Science Institute, Houston, 1986), pp. 411-424

J.E.P. Connerney, Planetary magnetism, in Planets and Moons, ed. by T. Spohn. Treatise on Geophysics, vol. 10 (Elsevier, Amsterdam, 2007), pp. 243-280 
J.E.P. Connerney, M.H. Acuña, P. Wasilewski, N.F. Ness, H. Rème, C. Mazelle, D. Vignes, R.P. Lin, D. Mitchell, P. Cloutier, Magnetic lineations in the ancient crust of Mars. Science 284, 794-798 (1999)

J.E.P. Connerney, M.H. Acuña, N.F. Ness, T. Spohn, G. Schubert, Mars crustal magnetism. Space Sci. Rev. 111(1-2), 1-32 (2004)

J.E.P. Connerney, M.H. Acuña, N.F. Ness, G. Kletetschka, D.L. Mitchell, R.P. Lin, H. Reme, From the cover: Tectonic implications of Mars crustal magnetism. Proc. Natl. Acad. Sci. 102, 14970-14975 (2005)

V. Conzelmann, Thermische Evolution des Planeten Merkur berechnet unter Anwendung verschiedener Viskositätsgesetze. Ph.D. Thesis, Westfälische Wilhelms-Universität Münster, 1999

S.O. Costin, S.L. Butler, Modelling the effects of internal heating in the core and lowermost mantle on the earth's magnetic history. Phys. Earth Planet. Inter. 157, 55-71 (2006)

F.J. Crary, F. Bagenal, Remanant ferromagnetism and the interior structure of Ganymede. J. Geophys. Res. 103, 25757-25773 (1998)

A. Davaille, C. Jaupart, Transient high-Rayleigh number thermal convection with large viscosity variations. J. Fluid Mech. 253, 141-166 (1993)

F. Deschamps, C. Sotin, Inversion of two-dimensional numerical convection experiments for a fluid with a strongly temperature-dependent viscosity. Geophys. J. Int. 143, 204-218 (2000)

J.O. Dickey, P.L. Bender, J.E. Faller et al., Lunar laser ranging: A continuing legacy of the Apollo program. Science 265, 482-490 (1994)

G. Dreibus, H. Wänke, Mars: A volatile-rich planet. Meteoritics 20, 367-382 (1985)

D.J. Dunlop, O. Ozdemir, Rock Magnetism: Fundamentals and Frontiers (Cambridge University Press, Cambridge, 1997), p. 573

A.M. Dziewonski, D.L. Anderson, Preliminary reference Earth model. Phys. Earth Planet. Inter. 25, 297-356 (1981)

L.T. Elkins-Tanton, E.M. Parmentier, P.C. Hess, Magma ocean fractional crystallization and cumulate overturn in terrestrial planets: Implications for Mars. Meteorit. Planet. Sci. 38(12), 1753-1771 (2003)

L.T. Elkins-Tanton, S. Zaranek, E.M. Parmentier, Early magnetic field and magmatic activity on Mars from magma ocean overturn. Earth Planet. Sci. Lett. 236, 1-12 (2005)

Y. Fei, C.T. Prewitt, H. Mao, C.M. Bertka, Structure and density of FeS at high pressure and high temperature and the internal structure of Mars. Science 268, 1892-1894 (1995)

Y. Fei, C.M. Bertka, L.W. Finger, High-pressure iron-sulfur compound, $\mathrm{Fe}_{3} \mathrm{~S}_{2}$, and melting relations in the Fe-FeS system. Science 275, 1621-1623 (1997)

Y. Fei, J. Li, C.M. Bertka, C.T. Prewitt, Structure type and bulk modulus of $\mathrm{Fe}_{3} \mathrm{~S}$, a new iron-sulfur compound. Am. Mineral. 85, 1830-1833 (2000)

W.M. Folkner, C.F. Yoder, D.N. Yuan, E.M. Standish, R.A. Preston, Interior structure and seasonal mass redistribution of Mars from radio tracking of Mars Pathfinder. Science 278, 1749-1752 (1997)

H.V. Frey, Impact constraints on the age and origin of the lowlands of Mars. Geophys. Res. Lett. 33, L08S02 (2006). doi:10.1029/2005GL024484

G. Giampieri, A. Balogh, Mercury's thermoelectric dynamo model revisited. Planet. Space Sci. 50, 757-762 (2002)

K.H. Glassmeier, H.-U. Auster, U. Motschmann, A feedback dynamo generating Mercury's magnetic field. Geophys. Res. Lett. 34, L22201 (2007). doi:10.1029/2007GL031662

F. Goarant, F. Guyot, J. Peyronneau, J.-P. Poirier, High-pressure and high-temperature reactions between silicates and liquid iron alloys, in the diamond anvil cell, studied by analytical electron microscopy. J. Geophys. Res. 97, 4477-4487 (1992)

O. Grasset, E.M. Parmentier, Thermal convection in a volumetrically heated, infinite Prandtl number fluid with strongly temperature-dependent viscosity: Implications for planetary thermal evolution. J. Geophys. Res. 103, 18171-18181 (1998)

O. Grasset, C. Sotin, F. Deschamps, On the internal structure and dynamics of Titan. Planet. Space Sci. 48, 617-636 (2000)

C. Grignè, S. Labrosse, P.J. Tackley, Convective heat transfer as a function of wavelength: Implications for the cooling of the Earth. J. Geophys. Res. 110, B03409 (2005). doi:10.1029/2004JB003376

M. Grott, D. Breuer, The evolution of the martian elastic lithosphere and implications for crustal and mantle rheology. Icarus 193, 503-515 (2008). doi:10.1016/j.icarus.2007.08.015

D. Gubbins, Energetics of the Earth's core. J. Geophys. 43, 453-464 (1977)

D. Gubbins, D. Alfë, G. Masters, D. Price, M.J. Gillan, Can the Earth's dynamo run on heat alone? Geophys. J. Int. 155, 609-622 (2003)

D. Gubbins, D. Alfë, G. Masters, D. Price, M.J. Gillan, Gross thermodynamics of 2-component core convection. Geophys. J. Int. 157, 1407-1414 (2004)

U. Hansen, D.A. Yuen, High Rayleigh number regime of temperature-dependent viscosity convection and the Earth's early thermal history. Geophys. Res. Lett. 20, 2191-2194 (1993)

H. Harder, U. Christensen, A one-plume model of Martian mantle convection. Nature 380, 507-509 (1996) 
H. Harder, G. Schubert, Sulfur in Mercury's core? Icarus 151, 118-122 (2001)

S.A. Hauck, R.J. Phillips, Thermal and crustal evolution of Mars. J. Geophys. Res. 107(E7) (2002). doi:10. 1029/2001JE001801

S.A. Hauck, A.J. Dombard, R.J. Phillips, S.C. Solomon, Internal and tectonic evolution of Mercury. Earth Planet. Sci. Lett. 222(3-4), 713-728 (2004)

S.A. Hauck, J.M. Aurnou, A.J. Dombard, Sulfur's impact on core evolution and magnetic field generation on Ganymede. J. Geophys. Res. 111 (2006)

J.W. Head, R. Greeley, M.P. Golombek et al., Geological processes and evolution. Space Sci. Rev. 96(1/4), 263-292 (2001)

M.H. Heimpel, J.M. Aurnou, F.M. Al-Shamali, N. Gomez Perez, A numerical study of dynamo action as a function of spherical shell geometry. Earth Planet. Sci. Lett. 236 (2005). doi:10.1016/j.epsl.2005.04.032

J.W. Hernlund, C. Thomas, P.J. Tackley, Phase boundary double crossing and the structure of Earth's deep mantle. Nature 434, 882-886 (2005). doi:10.1038/nature03472

J.M. Hewitt, D.P. McKenzie, N.O. Weiss, Dissipative heating in convective flows. J. Fluid Mech. 68, 721-738 (1975)

R. Hide, The hydrodynamics of the Earth's core. Phys. Chem. Earth 1, 94-137 (1956)

R. Hide, Comments on the Moon's magnetism. Moon 4, 39 (1972)

L. Hood, Z. Huang, Formation of magnetic anomalies antipodal to lunar impact basins: Two dimensional model calculations. J. Geophys. Res. 96, 9837-9846 (1991)

L.L. Hood, D.L. Mitchell, R.P. Lin, M.H. Acuna, A.B. Binder, Initial measurements of the lunar induced magnetic dipole moment using Lunar Prospector magnetometer data. Geophys. Res. Lett. 26, 23272330 (1999)

L. Hood, A. Vickery, Magnetic field amplification and generation in hypervelocity meteorid impacts with application to lunar paleomagnetism, in Proceedings of the Lunar and Planetary Science Conference 15th, Part 1. J. Geophys. Res. 89(supplement), C211-C223 (1984)

L.L. Hood, A. Zakharian, J. Halekas et al., Initial mapping and interpretation of lunar crustal magnetic anomalies using Lunar Prospector magnetometer data. J. Geophys. Res. 106, 27825-27839 (2001)

W.B. Hubbard, in Planetary Interiors (Van Nostrand-Reinhold, New York, 1984)

C. Huettig, Scaling laws for internally heated mantle convection, Ph.D. Thesis, Westfälische WilhelmsUniversität Münster, 2009

G. Hulot, C. Eymin, B. Langlais, M. Mandea, N. Olsen, Small-scale structure of the geodynamo inferred from Oersted and Magsat satellite data. Nature 416, 620-623 (2002)

H. Hussman, T. Spohn, Thermal-orbital evolution of Io and Europa. Icarus 171, 391-410 (2004)

H. Hussmann, C. Sotin, J.I. Lunine, Interiors and evolution of Icy satellites, in Planets and Moons, ed. by T. Spohn. Treatise on Geophysics, vol. 10 (Elsevier, Amsterdam, 2007), pp. 509-540

J.A. Jacobs, The Earth's inner core. Nature 172, 297-298 (1953)

C. Jaupart, S. Labrosse, J.-C. Mareschal, Temperatures, heat and energy in the mantle of the Earth, in Mantle Dynamics. Treatise of Geophysics, vol. 7 (Elsevier, Amsterdam, 2007), pp. 253-303

H. Jeffreys, The instability of a compressible fluid heated below. Proc. Camb. Philos. Soc. 26, 170-172 (1930)

C.A. Jones, Thermal and compositional convection in the outer core, in Core Dynamics, ed. by P. Olson. Treatise on Geophysics, vol. 8 (Elsevier, Amsterdam, 2007), pp. 131-185

S. Karato, D.C. Rubie, Towards an experimental study of deep mantle rheology: A new multi-anvil specimen assembly for deformation studies under high pressure and temperatures. J. Geophys. Res. 102, 20,11120,122 (1997)

S. Karato, P. Wu, Rheology of the upper mantle. Science 260, 771-778 (1993)

Y. Ke, V.S. Solomatov, Early transient superplumes and the origin of the Martian crustal dichotomy. J. Geophys. Res. 111 (2006). doi:10.1029/2005JE002631

T. Keller, P.J. Tackley, Towards self-consistent modelling of the Martian dichotomy: The influence of lowdegree convection on crustal thickness distribution. Icarus 202 (2009). doi:10.1016/j.icarus.2009.03.029

B.L.N. Kennett, E.R. Engdahl, R.P. Buland, Constraints on seismic velocities in the Earth from travel times. Geophys. J. Int. 122, 108-124 (1995)

R. Kerswell, Elliptical instability. Ann. Rev. Fluid Mech. 34, 83-113 (2002)

A. Khan, K. Mosegaard, J.G. Williams, P. Lognonné, Does the Moon possess a molten core? Probing the deep lunar interior using results from LLR and Lunar Prospector. J. Geophys. Res. 109(E09), E09007 (2004). doi:10.1029/2004JE002294

J. Kimura, T. Nakagawa, K. Kurita, Size and compositional constraints of Ganymede's metallic core for driving an active dynamo. Icarus (2009). doi:10.1016/-j.icarus.2009.02.026

R.L. Kirk, D.J. Stevenson, Thermal evolution of a differentiated Ganymede and implications for surface features. Icarus 69, 91-134 (1987)

M.G. Kivelson, K.K. Khurana, C.T. Russell, R.J. Walker, J. Warnecke, F.V. Coroniti, C. Polanskey, D.J. Southwood, G. Schubert, Discovery of Ganymede's magnetic field by the Galileo Spacecraft. Nature 384, 537-541 (1996) 
M.G. Kivelson, K.K. Khurana, M. Volwerk, The permanent and inductive magnetic moments of Ganymede. Icarus 157, 507-522 (2002)

E. Knittle, R. Jeanloz, Earth's core-mantle boundary: Results of experiments at high pressures and temperatures. Science 251, 1438-1443 (1991)

A.S. Konopliv, S.W. Asmar, E. Carranza, W.L. Sjogren, D.N. Yuan, Recent gravity models as a result of the Lunar Prospector mission. Icarus 150, 1-18 (2001)

A.S. Konopliv, C.F. Yoder, E.M. Standish, D.N. Yuan, W.L. Sjogren, A global solution for the Mars static and seasonal gravity, Mars orientation, Phobos and Deimos masses, and Mars ephemeris. Icarus 182, 23-50 (2006)

W. Konrad, T. Spohn, Thermal history of the Moon: Implications for an early core dynamo and postaccretional magmatism. Adv. Space Res. 19(10), 1511-1521 (1997)

J. Korenaga, Archean geodynamics and the thermal evolution of the Earth, in Archean Geodynamics and Environments, ed. by K. Benn, J. Mareschal, K.C. Condie. Geophysical Monograph Series, vol. 164 (American Geophysical Union, Washington, 2006), pp. 7-32

Y.N. Kulikov, H. Lammer, H.I.M. Lichtenegger, T. Penz, D. Breuer, T. Spohn, R. Lundin, H.K. Biernat, A comparative study of the influence of the active young sun on the early atmospheres of Earth, Venus, and Mars. Space Sci. Rev. 129(1-3), 207-243 (2007). doi:10.1007/s11214-007-9192-4

O.L. Kuskov, V.A. Kronrod, Core sizes and internal structure of Earth's and Jupiter's satellites. Icarus 151, 204-227 (2001)

S. Labrosse, Hotspots, mantle plumes and core heat loss. Earth Planet. Sci. Lett. 199, 147-156 (2002)

S. Labrosse, Thermal and magnetic evolution of the Earth's core. Phys. Earth Planet. Inter. 140, 127-143 (2003)

S. Labrosse, C. Jaupart, Thermal evolution of the earth: Secular changes and fluctuations of plate characteristics. Earth Planet. Sci. Lett. 260, 465-481 (2007)

S. Labrosse, J.-P. Poirier, J.-L. Le Mouîl, On cooling of the Earth's core. Phys. Earth Planet. Inter. 99, 1-17 (1997)

S. Labrosse, J.-P. Poirier, J.-L. Le Mouîl, The age of the inner core. Earth Planet. Sci. Lett. 190, 111-123 (2001)

S. Labrosse, J.W. Hernlund, N. Coltice, A crystallizing dense magma ocean at the base of Earth's mantle. Nature 450, 866-869 (2007)

A. Landolt-Börnstein, Terrestrial planets and satellites: Planetary interiors, in Numerical Data and Functional Relationships in Science and Technology. New Series Group VI: Astronomy and Astrophysics, 4: Astronomy, Astrophysics, and Cosmology (Subvolume B Solar System) (Springer, Berlin, 2009), pp. 200-224. ISBN 978-3-540-88054-7. ISSN 0942-8011

B. Langlais, M.E. Purucker, M. Mandea, Crustal magnetic field of Mars. J. Geophys. Res. 109, 37 (2004). doi:10.1029/2003JE002048

B. Langlais, V. Lesur, M.E. Purucker, J.P.E. Connerney, M. Mandea, Crustal magnetic fields of terrestrial planets. Space Sci. Rev. (2009). doi:10.1007/s11214-009-9557-y, this issue

T. Lay, J. Hernlund, B.A. Buffett, Core-mantle boundary heat flow. Nat. Geosci. 1, 25-32 (2008)

F.G. Lemoine, D.E. Smith, D.D. Rowlands et al., An improved solution of the gravity field of Mars (GMM2B) from Mars Global Surveyor. J. Geophys. Res. 106, 23359-23376 (2001)

J.S. Lewis, The temperature gradient in the solar nebula. Science 186, 440-443 (1972)

J.S. Lewis, Io: Geochemistry of sulfur. Icarus 50, 103-114 (1982)

J.S. Lewis, Origin and composition of Mercury, in Mercury, ed. by F. Vilas, C.R. Chapman, M.S. Matthews (Univ. of Arizona Press, Tucson, 1988), pp. 651-666

J. Li, C.B. Agee, Geochemistry of mantle-core differentiation at high pressure. Nature 381, 686-689 (1996)

J.R. Lister, Expressions for the dissipation driven by convection in the Earth's core. Phys. Earth Planet. Inter. 140, 145-158 (2003)

J.R. Lister, B.A. Buffett, The strength and efficiency of the thermal and compositional convection in the geodynamo. Phys. Earth Planet. Inter. 91, 17-30 (1995)

J.R. Lister, B.A. Buffett, Stratification of the outer core at the core-mantle boundary. Phys. Earth Planet. Inter. 105, 5-19 (1998)

K. Lodders, B. Fegley, The Planetary Scientist's Companion (Oxford University Press, Oxford, 1998)

P. Lognonne, C. Johnson, Planetary seismology, in Planets and Moons, ed. by T. Spohn. Treatise on Geophysics, vol. 10 (Elsevier, Amsterdam, 2007), pp. 69-122

D.E. Loper, The gravitationally powered dynamo. Geophys. J. R. Astron. Soc. 54, 389-404 (1978a)

D.E. Loper, Some thermal consequences of a gravitationally powered dynamo. J. Geophys. Res. 83, 59615970 (1978b)

J.I. Lunine, D.J. Stevenson, Clathrate and ammonia hydrates at high pressure: Application to the origin of methane on Titan. Icarus 70, 61-77 (1987) 
J.L. Margot, S.J. Peale, R.F. Jurgens, M.A. Slade, I.V. Holin, Large longitude libration of mercury reveals a molten core. Science 316(5825), 710-714 (2007)

W.B. McKinnon, Core evolution in the icy satellites, and the prospects for dynamo-generated magnetic fields. Bull. Am. Astron. Soc. 28, 1076 (1996)

W.B. McKinnon, K. Zahnle, B.A. Ivanov, H.J. Melosh, Cratering on Venus: Modeling and observations, in Venus II, ed. by S.W. Bougher, D.M. Hunten, R.J. Phillips (University of Arizona Press, Tucson, 1997), pp. $969-1014$

H.Y. McSween Jr., SNC meteorites: Clues to Martian petrologic evolution? Rev. Geophys. 23, 391-416 (1985)

D.L. Mitchell, R.P. Lin, H. Rème, M.H. Acuña, P.A. Cloutier, N.F. Ness, Crystal magnetospheres observed in the Martian night hemisphere, in American Astronomical Society, DPS Meeting \#31, abstracts \#59.04, Bull. Am. Astron. Soc. 31, 1584 (1999)

D.L. Mitchell, R.P. Lin, C. Mazelle, H. Rème, P.A. Cloutier, J.E.P. Connerney, M.H. Acuña, N.F. Ness, Probing Mars' crustal magnetic field and ionosphere with the MGS electron reflectometer. J. Geophys. Res. 106(E10), 23,419-23,428 (2001)

D.L. Mitchell, J.S. Halekas, R.P. Lin, S. Frey, L.L. Hood, M.H. Acuña, Global mapping of lunar crustal fields by Lunar Prospector. Icarus 194, 401-409 (2008)

P.S. Mohit, J. Arkani-Hamed, Impact demagnetization of the Martian crust. Icarus 168 (2004). doi:10.1016/ j.icarus.2003.12.005

L.-N. Moresi, V.S. Solomatov, Numerical investigation of 2D convection with extremely large viscosity variations. Phys. Fluids 7, 2154-2162 (1995)

S. Mollett, Thermal and magnetic constraints on the cooling of the Earth. Geophys. J. R. Astron. Soc. 76, 653-666 (1984)

W.B. Moore, Heat transport in a convecting layer heated from within and below. J. Geophys. Res. 113 (2008)

K. Nagel, D. Breuer, T. Spohn, A model for the interior structure, evolution, and differentiation of Callisto. Icarus 169, 402-412 (2004)

T. Nakagawa, P.J. Tackley, Effects of thermo-chemical mantle convection on the thermal evolution of the Earth's core. Earth Planet. Sci. Lett. 220, 107-119 (2004)

T. Nakagawa, P.J. Tackley, Deep mantle heat flow and thermal evolution of the Earth's core in thermochemical multiphase models of mantle convection. Geochem. Geophys. Geosyst. 6, Q08003 (2005). doi:10.1029/ 2005GC000967

Y. Nakamura, G. Latham, D. Lammlein, M. Ewing, F. Duennebier, J. Dorman, Deep lunar interior inferred from recent seismic data. Geophys. Res. Lett. 1, 137-140 (1974)

N.F. Ness, K.W. Behannon, R.P. Lepping, Y.C. Whang, Magnetic field of Mercury confirmed. Nature 255, 204 (1975)

N.F. Ness, M.H. Acuña, J. Connerney, P. Wasilewski, C. Mazelle, J. Sauvaud, D. Vignes, C. D’Uston, H. Reme, R. Lin, D.L. Mitchell, J. McFadden, D. Curtis, P. Cloutier, S.J. Bauer, MGS magnetic fields and electron reflectometer investigation: Discovery of paleomagnetic fields due to crustal remanence. Adv. Space Res. 23(11), 1879-1886 (1999)

F. Nimmo, Energetics of the core, in Treatise on Geophysics, vol. 8, ed. by G. Schubert (Elsevier, Amsterdam, 2007), pp. 31-66

F. Nimmo, D. Stevenson, Influence of early plate tectonics on the thermal evolution and magnetic field of Mars. J. Geophys. Res. 105, 11,969-11,979 (2000)

F. Nimmo, G.D. Price, J. Brodholt, D. Gubbins, The influence of potassium on core and geodynamo evolution. Geophys. J. Int. 156, 263-376 (2004)

P. Olson, Overview on core dynamics, in Treatise on Geophysics, vol. 8, ed. by G. Schubert (Elsevier, Amsterdam, 2007), pp. 1-31. Chap. 8.01

C. O’Neill, A. Lenardic, Geological consequences of super-sized Earths. Geophys. Res. Lett. 34, L19204 (2007). doi:10.1029/2007GL030598

H. Ozawa, K. Hirose, M. Mitome, Y. Bando, N. Sata, Y. Ohishi, Chemical equilibrium between ferropericlase and molten iron to $134 \mathrm{GPa}$ and implications for iron content at the bottom of the mantle. Geophys. Res. Lett. 35 (2008)

M. Ozima, K. Seki, N. Terada, Y.N. Miura, F.A. Podosek, H. Shinagawa, Terrestrial nitrogen and noble gases in lunar soils. Nature 436, 655-659 (2005)

E.M. Parmentier, S. Zhong, M. Zuber, Gravitational differentiation due to initial chemical stratification: Origin of lunar asymmetry by the creep of denseKREEP? Earth Planet Sci. Lett. 201, 473-480 (2002)

S.J. Peale, Inferences from the dynamical history of Mercury's rotation. Icarus 28, 459-467 (1976)

S.J. Peale, The rotational dynamics of Mercury and the state of its core, in Mercury, ed. by F. Vilas, C.R. Chapman, M.S. Matthews (University of Arizona Press, Tucson, 1988), pp. 461-493

J.-P. Poirier, Transport properties of liquid metals and viscosity of the Earth's core. Phys. Earth Planet. Inter. 92, 99-105 (1988) 
J.-P. Poirier, Light elements in the Earth's core: A critical review. Phys. Earth Planet. Inter. 85, 319-337 (1994)

M.E. Purucker, A global model of the internal magnetic field of the Moon based on Lunar Prospector magnetometer observations. Icarus 197 (2008). doi:10.1016/j.icarus.2008.03.016

N.J. Rappaport, A.S. Konopliv, A.B. Kucinskas, P.G. Ford, An improved 360 degree and order model of Venus topography. Icarus 139, 19-31 (1999)

C.C. Reese, V.S. Solomatov, J.R. Baumgardner, Scaling laws for time dependent stagnant lid convection in a spherical shell. Phys. Earth Planet. Inter. 149, 361-370 (2005)

G.O. Roberts, Fast viscous Bernard convection. Geophys. Astrophys. Fluid Dyn. 12, 235-272 (1979)

P.H. Roberts, Theory of the geodynamo, in Core Dynamics, ed. by P. Olson. Treatise on Geophysics, vol. 8 (Elsevier, Amsterdam, 2007), pp. 67-105

J.H. Roberts, S. Zhong, Degree-1 convection in the Martian mantle and the origin of the hemisphere dichotomy. J. Geophys. Res. 111, E06013 (2006)

J.H. Roberts, R.J. Lillis, M. Manga, Giant impacts on early Mars and the cessation of the Martian dynamo. J. Geophys. Res. 114, E04009 (2009). doi:10.1029/2008JE003287

P.H. Roberts, C.A. Jones, A.R. Calderwood, Energy fluxes and ohmic dissipation in the Earth's core, in Earth's Core and Lower Mantle, ed. by C.A. Jones, A.M. Soward, K. Zhang (Taylor \& Francis, London, 2003), pp. 100-129

P. Rochette, G. Fillion, R. Ballou, F. Brunet, B. Ouladdiaf, L.L. Hood, High pressure magnetic transition in pyrrhotite and impact demagnetization on Mars. Geophys. Res. Lett. 30 (2003). doi:10.1029/ 2003GL017359

S.K. Runcorn, An ancient lunar magnetic dipole field. Nature 253, 701-703 (1975)

C.T. Russell, R.C. Elphic, J.A. Slavin, Initial Pioneer Venus magnetic field results: Dayside observations. Science 203, 745 (1979a)

C.T. Russell, R.C. Elphic, J.A. Slavin, Initial Pioneer Venus magnetic field results: Nightside observations. Science 205, 114 (1979b)

C. Sanloup, A. Jambon, P. Gillet, A simple chondritic model of Mars. Phys. Earth Planet. Inter. 112, 43-54 (1999)

G.R. Sarson, C.A. Jones, K. Zhang, G. Schubert, Magneto-convection dynamos and the magnetic fields of Io and Ganymede. Science 276, 1106-1108 (1997)

G.G. Schaber, R.G. Strom, H.J. Moore et al., Geology and distribution of impact craters on Venus: What are they telling us? J. Geophys. Res. 97, 13,257-13,301 (1992)

G. Schubert, Ann. Rev. Earth Planet. Sci. 7, 289 (1979)

G. Schubert, T. Spohn, Thermal history of Mars and the sulfur content of its core. J. Geophys. Res. 95, 14095-14104 (1990)

G. Schubert, P. Cassen, R.E. Young, Subsolidus convective cooling histories of terrestrial planets. Icarus 38, 192-211 (1979)

G. Schubert, M.N. Ross, D.J. Stevenson, T. Spohn, Mercury's thermal history and the generation of its magnetic field, in Mercury, ed. by F. Viulas, C.R. Chapman, M.S. Matthews (Univ. Press of Arizona, Tuscon, 1988), pp. 514-561

G. Schubert, S.C. Solomon, D.L. Turcotte, M.J. Drake, N.H. Sleep, Origin and thermal evolution of Mars, in Mars, ed. by H.H. Kieffer, B.M. Jakosky, C.W. Snyder, M.S. Matthews (Univ. Press of Arizona, Tucson, 1992), pp. 147-183

G. Schubert, K. Zhang, M.G. Kivelson, J.D. Anderson, The magnetic field and internal structure of Ganymede. Nature 384, 544-545 (1996)

G. Schubert, C.T. Russell, W.B. Moore, Timing of the Martian dynamo. Nature 408, 666-667 (2000)

G. Schubert, D.L. Turcotte, P. Olson, Mantle Convection in the Earth and Planets (Cambridge Univ. Press, Cambridge, 2001), 940 pp.

G. Schubert, J.D. Anderson, T. Spohn, W.B. McKinnon, Interior composition, structure and dynamics of the Galilean satellites, in Jupiter, ed. by F. Bagnell, T. Dowling, W.B. McKinnon. The Planet, Satellites and Magnetosphere (Cambridge University Press, Cambridge, 2004), pp. 281-306

S. Schumacher, D. Breuer, Influence of a variable thermal conductivity on the thermochemical evolution of Mars. J. Geophys. Res. 111, E02006 (2006). doi:10.1029/2005JE002429

H.P. Scott, Q. Williams, F.J. Ryerson, Experimental constraints on the chemical evolution of large icy satellites. Earth Planet. Sci. Lett. 203, 399-412 (2002)

H.N. Sharpe, W.R. Peltier, Parameterized mantle convection and the Earth's thermal history. Geophys. Res. Lett. 5, 737-740 (1978)

H.N. Sharpe, W.R. Peltier, A thermal history model for the Earth with parameterized convection. Geophys. J. R. Astron. Soc. 59, 171-203 (1979)

A.P. Showman, R. Malhotra, Tidal evolution into the Laplace resonance and the resurfacing of Ganymede. Icarus 127, 93-111 (1997) 
A.P. Showman, D.J. Stevenson, R. Malhotra, Coupled orbital and thermal evolution of Ganymede. Icarus 129, 367-383 (1997)

R.W. Siegfried, S.C. Solomon, Icarus 23, 192-205 (1974)

W.L. Sjogren, W.B. Banerdt, P.W. Chodas et al., The Venus gravity field and other geodetic parameters, in Venus II: Geology, Geophysics, Atmosphere, and Solar Wind Environment, ed. by S.W. Bougher, D.M. Hunten, R.J. Philips (Univ. Press of Arizona, Tucson, 1997), pp. 1125-1162

D.E. Smith, M.T. Zuber, The shape of Mars and the topographic signature of the hemispheric dichotomy. Science 271, 184-188 (1996)

F. Sohl, G. Schubert, Interior structure, composition, and mineralogy of the terrestrial planets, in Planets and Moons, ed. by T. Spohn. Treatise on Geophysics, vol. 10 (Elsevier, Amsterdam, 2007), pp. 27-68

F. Sohl, T. Spohn, The structure of Mars: Implications from SNC-Meteorites. J. Geophys. Res. 102, 16131635 (1997)

F. Sohl, T. Spohn, D. Breuer, K. Nagel, Implications from Galileo observations on the interior structure and chemistry of the Galilean satellites. Icarus 157, 104-119 (2002)

F. Sohl, G. Schubert, T. Spohn, Geophysical constraints on the composition and structure of the Martian interior. J. Geophys. Res. 110, E12008 (2005). doi:10.1029/2005JE002520

V.S. Solomatov, Scaling of temperature- and stress-dependent viscosity. Phys. Fluids 7, 266-274 (1995)

V.S. Solomatov, L.N. Moresi, Scaling of time-dependent stagnant lid convection: Application to small-scale convection on Earth and other terrestrial planets. J. Geophys. Res. 105, 21795-21817 (2000)

S.C. Solomon, R.L. McNutt Jr., R.E. Gold, M.H. Acuña, D.N. Baker, W.V. Boynton, C.R. Chapman, A.F. Cheng, G. Gloeckler, J.W. Head III, S.M. Krimigis, W.E. McClintock, S.L. Murchie, S.J. Peale, R.J. Phillips, M.S. Robinson, J.A. Slavin, D.E. Smith, R.G. Strom, J.I. Trombka, M.T. Zuber, Planet. Space Sci. 49, 1445-1465 (2001)

C.S. Solomon, O. Aharonson, J.M. Aurnou et al., New perspectives on ancient Mars. Science 307, 1214-1220 (2005)

A. Souriau, Deep Earth structure-The Earth's cores, in Seismology and the Structure of the Earth, ed. by A.M. Dziewonski, B.A. Romanowicz. Treatise on Geophysics, vol. 1 (Elsevier, Amsterdam, 2007), pp. $655-693$

E.A. Spiegel, G. Veronis, On the Boussinesq approximation for a compressible fluid. Astrophys. J. 131, 442447 (1960)

T. Spohn, Mantle differentiation and thermal evolution of Mars, Mercury, and Venus. Icarus 90, 222-236 (1991)

T. Spohn, D. Breuer, Implications from Galileo observations on the interior structure and evolution of the Galilean satellites, in Planetary Systems: The Long View, ed. by L.M. Celnikier, J. Tran Thanh Van (Editions Frontiers, 1998), pp. 135-145

T. Spohn, F. Sohl, D. Breuer, Mars. Astron. Astrophys. Rev. 8, 181-235 (1998)

T. Spohn, F. Sohl, K. Wieczerkowski, V. Conzelmann, The interior structure of Mercury: What we know, what we expect from BepiColombo. Planet. Space Sci. 49, 1561-1570 (2001a)

T. Spohn, M.A. Acunã, D. Breuer et al., Geophysical constraints on the evolution of Mars. Space Sci. Rev. 96, 231-262 (2001b)

T. Spohn, W. Konrad, D. Breuer, R. Ziethe, Lunar volcanism induced by heat advected from the lower mantle: Results of 2D and 3D mantle convection calculations. Icarus 149, 54-65 (2001c)

F.D. Stacey, O.L. Anderson, Electrical and thermal conductivities of Fe-Ni-Si alloy under core conditions. Phys. Earth Planet. Inter. 124, 153-162 (2001)

S. Stanley, Glatzmeier, Dynamo models for planets other than Earth. Space Sci. Rev. (2009). doi:10.1007/ s11214-009-9573-y, this issue

S. Stanley, J. Bloxham, W.E. Hutchinson, M.T. Zuber, Thin shell dynamo models consistent with Mercury's weak observed magnetic field. Earth Planet. Sci. Lett. 234, 27-38 (2005). doi:10.1016/j.eps1.2005.02. 040

S. Stanley, L. Elkins-Tanton, M.T. Zuber, E.M. Parmentier, Mars' paleomagnetic field as the result of a single-hemisphere dynamo. Science 321(5897), 1822-1825 (2008)

D.R. Stegman, A.M. Jellinek, S.A. Zatman, J.R. Baumgardner, M.A. Richards, An early lunar core dynamo driven by thermochemical mantle convection. Nature 421, 143-146 (2003a)

V. Steinbach, D. Yuen, Effects of depth-dependent properties on the thermal anomalies produced in flush instabilities from phase transitions. Phys. Earth Planet. Inter. 86, 165-183 (1994)

D.R. Stegman, A.M. Jellinek, S.A. Zatman, J.R. Baumgardner, M.A. Richards, An early lunar core dynamo driven by thermochemical mantle convection. Nature 421(6919), 143-146 (2003b)

A. Stephenson, S.K. Runcorn, D.W. Collinson, On changes in the intensity of the ancient lunar magnetic field, in Proceedings of the 6th Lunar Science Conference. Geochim. Cosmochim. Acta (1975)

D.J. Stevenson, Planetary magnetic fields. Rep. Prog. Phys. 46, 555-620 (1983). doi:10.1088/0034-4885/46/ $5 / 001$ 
D.J. Stevenson, Mercury's magnetic field: A thermoelectric dynamo? Earth Planet. Sci. Lett. 82, 114 (1987)

D.J. Stevenson, When Galileo met Ganymede. Nature 384, 511-512 (1996)

D.J. Stevenson, Mars core and magnetism. Nature 412, 214-219 (2001)

D.J. Stevenson, Possible connections between the history of the Venus magnetic field and observable features, American Geophysical Union, Spring Meeting 2002, abstract \#P21A-08 (2002)

D. Stevenson, T. Spohn, G. Schubert, Magnetism and thermal evolution of the terrestrial planets. Icarus 54, 466-489 (1983)

A.J. Stewart, M.W. Schmidt, W. van Westrenen, C. Liebske, Mars: A new core-crystallization regime. Science 316, 1323-1325 (2007)

R.G. Strom, N.J. Trask, J.E. Guest, J. Geophys. Res. 80, 2478-2507 (1975)

P.J. Tackley, The quest for self-consistent incorporation of plate tectonics in mantle convection, in History and Dynamics of Global Plate Motions, ed. by M.A. Richards, R. Gordon, R. van der Hilst. Geophysical Monograph Series, vol. 121 (American Geophysical Union, Washington, 2000a)

P.J. Tackley, Self-consistent generation of tectonic plates in time-dependent, three-dimensional mantle convection simulations, Part 1: Pseudoplastic yielding. Geochem. Geophys. Geosyst. 1 (2000b)

J.A. Tarduno, R.D. Cottrell, M.K. Watkeys, D. Bauch, Geomagnetic field strength 3.2 billion years ago recorded by single silicate crystals. Nature 446, 657-660 (2007)

H. Terasaki, T. Kato, S. Urakawa, K. Funakoshi, A. Suzuki, T. Okada, M. Maeda, J. Sato, T. Kubo, S. Kasai, The effect of temperature, pressure, and sulfur content on viscosity of the Fe-FeS melt. Earth Planet. Sci. Lett. 190, 93-101 (2001)

Y. Thibault, M.J. Walter, The influence of pressure and temperature on the metal-silicate partition coefficients of nickel and cobalt in a model $\mathrm{C} 1$ chondrite and implications for metal segregation in a deep matma ocean. Geochim. Cosmochim. Acta 59, 991-1002 (1995)

A. Tilgner, Precession driven dynamos. Phys. Fluids 17, 034104 (2005)

G. Tobie, A. Mocquet, C. Sotin, Tidal dissipation within large icy satellites: Europa and Titan. Icarus 177, 534-549 (2005)

P.B. Toft, J. Arkani-Hamed, Magnetization of the Pacific Ocean lithosphere deduced from Magsat data. J. Geophys. Res. 97, 4387-4406 (1992)

R.A. Trompert, U. Hansen, On the Rayleigh number dependence of convection with strongly temperature dependent viscosity. Phys. Fluids 10(2), 351-360 (1998)

D.L. Turcotte, An episodic hypothesis for Venusian tectonics. J. Geophys. Res. 98, 17,061-17,068 (1993)

D.L. Turcotte, E.R. Oxburgh, Finite amplitude convective cells and continental drift. J. Fluid. Mech. 28, 29-42 (1967)

D. Valencia, R.J. O'Connell, Convection scaling and subduction on Earth and super-Earths. Earth Planet. Sci. Lett. 286, 492-502 (2009)

D. Valencia, R.J. O'Connell, D. Sasselov, Inevitability of plate tectonic on super-Earths. Astrophys. J. 670, L45-L48 (2007)

T. Van Holst, The rotation of the terrestrial planets, in Planets and Moons, ed. by T. Spohn. Treatise on Geophysics, vol. 10 (Elsevier, Amsterdam, 2007), pp. 123-164

L. Vocadlo, Mineralogy of the Earth-The Earth's core: Iron and iron alloys, in Mineral Physics, ed. by D. Price. Treatise on Geophysics, vol. 2 (Elsevier, Amsterdam, 2007), pp. 91-120

S.A. Weinstein, The effects of a deep mantle endothermic phase change on the structure of thermal convection in silicate planets. J. Geophys. Res. 100(E6), 11,719-11,728 (1995)

B. Weiss, J. Berdahl, L. Elkins-Tanton, S. Stanley, E.A. Lima, L. Carpozen, Magnetism on the angrite parent body and the early differentiation of planetesimals. Science 332, 713-716 (2008)

G.W. Wetherill, Acculmulation of mercury from planetesimals, in Mercury, ed. by F. Viulas, C.R. Chapman, M.S. Matthews (Univ. Press of Arizona, Tucson, 1988), pp. 514-561

M.A. Wieczorek, Gravity and topography of the terrestrial planets, in Planets and Moons, ed. by T. Spohn. Treatise on Geophysics, vol. 10 (Elsevier, Amsterdam, 2007), pp. 165-206

U. Wienbruch, T. Spohn, A self sustained magnetic field on Io? Planet. Space Sci. 9, 1045-1057 (1995)

J.G. Williams, D.H. Boggs, C.F. Yoder, J.T. Ratcliff, J.O. Dickey, Lunar rotational dissipation in solid body and molten core. J. Geophys. Res. 106, 27,933-27,968 (2001)

J.-P. Williams, F. Nimmo, Thermal evolution of the Martian core: Implications for an early dynamo. Geology 32, 97-100 (2004)

D.T. Woods, J.M. Shull, C.L. Sarazin, Astrophys. J. 249, 399 (1981)

S. Xie, P.J. Tackley, Evolution of helium and argon isotopes in a convecting mantle. Phys. Earth Planet. Inter. 146, 417-439 (2004)

C.F. Yoder, The free librations of a dissipative Moon. Philos. Trans. R. Soc. Lond. Ser. A 303, 327-338 (1981)

C.F. Yoder, Venusian spin dynamics, in Venus II: Geology, Geophysics, Atmosphere, and Solar Wind Environment, ed. by S.W. Bougher, D.M. Hunten, R.J. Philips (Univ. Press of Arizona, Tucson, 1997), pp. $1087-1124$ 
C.F. Yoder, A.S. Konopliv, D.N. Yuan, E.M. Standish, W.M. Folkner, Fluid core size of Mars from detection of the solar tide (1993). doi:10.1126/science.1079645

C.F. Yoder, A.S. Konopliv, D.N. Yuan, E.M. Standish, W.M. Folkner, Fluid core size of Mars from detection of the solar tide. Science 300, 299-303 (2003). doi:10.1126/science.1079645

T. Yukutake, Implausibility of thermal convection in the Earth's solid inner core. Phys. Earth Planet. Inter. 108, 1-13 (1998)

R. Ziethe, K. Seiferlin, H. Hiesinger, Duration and extent of lunar volcanism: Comparison of 3D convection models to mare basalt ages. Planet. Space Sci. 57(7), 784-796 (2009). doi:10.1016/j.pss.2009.02.002

V.N. Zharkov, V.P. Trubitsyn, Physics of Planetary Interiors (Pachart, Tucson, 1978)

S. Zhong, M.T. Zuber, Degree-1 mantle convection and the crustal dichotomy on Mars. Earth Planet. Sci. Lett. 189, 75-84 (2001)

M.T. Zuber, S.C. Solomon, R.J. Phillips et al., Internal structure and early thermal evolution of Mars from Mars Global Surveyor topography and gravity. Science 287, 1788-1793 (2000) 